hep-th/0406225

UT-KOMABA/04-8

June 2004

\title{
Resolving the Holography in the Plane-Wave Limit of AdS/CFT Correspondence
}

\author{
Suguru Dobashi * and Tamiaki Yoneya ${ }^{\dagger}$ \\ Institute of Physics, University of Tokyo \\ Komaba, Meguro-ku, Tokyo 153-8902
}

\begin{abstract}
The issue of holographic mapping between bulk and boundary in the plane-wave limit of AdS/SYM correspondence is reexamined from the viewpoint of correlation functions. We first study the limit of large angular momentum for the so-called GKP-W relation in supergeravity approximation, connecting directly the effective action in the bulk and the generating functional of correlation functions on the boundary. The spacetime tunneling picture which has been proposed in our previous works naturally emerges. This gives not only a justification of our previous proposal, with some important refinements, on the mapping between bulk effective interaction and the CFT coefficients on the boundary in the plane-wave limit, but also implies various insights on the interpretation of holography in the plane-wave limit. Based on this result, we construct a new 'holographic' string field theory. We confirm for several nontrivial examples that this gives the CFT coefficients derived by perturbation theory on the gauge-theory side. Our results are useful for understanding how apparently different duality maps proposed from different standpoints are consistent with each other and with our definite spacetime picture for the AdS holography in the plane-wave limit.
\end{abstract}

\footnotetext{
*e-mail address: doba@hep1.c.u-tokyo.ac.jp

${ }^{\dagger}$ e-mail address: tam@hep1.c.u-tokyo.ac.jp
} 


\section{Introduction}

An impressive amount of computations have been done following the BMN conjecture [1] as to the identification of stringy operators in AdS/CFT correspondence. In spite of all those important works of two years, however, it seems that the question of holographic correspondence of correlation functions for the BMN operators still has not been appropriately understood. In the case of the original $\mathrm{AdS}_{5} / \mathrm{SYM}_{4}$ correspondence, the relation between the bulk fields $\left\{\phi_{i}(z, \vec{x})\right\}$ and the gauge-invariant operators $\left\{O_{i}(\vec{x})\right\}$ of 4D Yang-Mills theory has been concretely formulated as the famous GKP-W relation [2]

$$
Z[\phi] \sim\left\langle\exp \left[\int d^{4} x \sum_{i} \phi_{i}(\vec{x}) O_{i}(\vec{x})\right]\right\rangle
$$

which connects the boundary values $\lim _{z \rightarrow 0} z^{\Delta_{i}-4} \phi_{i}(z, \vec{x})=\phi_{i}(\vec{x})$ of the bulk fields to the source fields coupled with $\left\{\mathcal{O}_{i}(\vec{x})\right\}$ at the conformal boundary of AdS spacetime. If one naively followed the Penrose limit in the bulk of AdS spacetime in obtaining a plane-wave approximation, one would end up in a puzzling situation that the plane-wave geometry corresponding to the large angular momentum along a direction of $S^{5}$ cannot be related to the conformal boundary, since the null trajectory adopted by the BMN proposal never reaches the conformal boundary. Because of this difficulty, some different ways of comparing both sides without relying directly upon the GKP-W relation have been discussed in the literature [3]. However, lacking for more direct links to physical observables, these proposals seem to be still regarded as phenomenological data towards a better understanding of holography. It is very important to resolve the issue of holographic correspondence from the viewpoint of correlation functions, since it would be a crucial basis in addressing physically more relevant questions related to the duality between closed string theories and gauge theories.

In previous works, we have presented basic ideas on a possible reconciliation of the BMN proposal with the GKP-W relation. In ref. [4] [5], we proposed to interpret the GKP-W relation in the plane-wave limit as a consequence of the tunneling propagation of the BMN states of closed strings from boundary to boundary. The motivation for our proposal was puzzles which arises in connection of holography when we adopt seemingly familiar premises in the literature, especially, the identification of the global AdS time (or light-cone time) with the time of radial quantization on the CFT side. ${ }^{\ddagger}$ We have

\footnotetext{
${ }^{\ddagger}$ In our opinion, the origin of a puzzle discussed in [6] is also related to this identification. For a list
} 
argued that these puzzles are resolved, by considering the tunneling trajectory connecting AdS boundary to AdS boundary. Since the role of time parameter is played by the affine parameter along the tunneling trajectory which is orthogonal to the conformal boundary, we cannot identify it with radial time directly, and consequently all the puzzles are naturally resolved. In subsequent works [7, our ideas have been extended successfully to a more general nonconformal case of $\mathrm{D} p$-brane backgrounds, by deriving the generalized correspondence [8] obtained previously between nonconformal SYM theories and D $p$-brane backgrounds.

However, our original argument in [4] has not yet been completely satisfactory, since it involves some ambiguities with respect to normalization and short-distance cutoff when we discuss 3-point and higher correlation functions. One of the purposes of the present work is to reexamine our basic ideas from an equivalent but a more systematic standpoint by studying the plane-wave limit directly on the GKP-W relation, and to strengthen our picture by presenting further supports and extensions. In our first work [4, we have not started from the GKP-W relation. Instead, we have treated the bulk field equation in the WKB approximation and then proposed a natural ansatz for relating correlation functions and the Euclidean S-matrix in the same spirit as the GKP-W relation. In the present work, we study the limit of large R-charge $(J)$ for the GKP-W relation directly and confirm that our original picture emerges automatically within the supergravity approximation. Then, on the basis of the known relation [11] for chiral operators between supergravity and SYM gauge theory, we establish a definite relation between 3-point correlators and the bulk effective interactions which are consistent with our original picture, including precise normalization and cutoff.

It turns out that the resultant supergravity effective theory in the plane-wave limit cannot be obtained from any versions of previously known string field theories. Our result can be adopted as a strong constraint in constructing a string field theory describing higher stringy modes in accordance with our picture. We propose a new 'holographic' string field theory, which reduces to the derived effective action when restricted in the supergravity sector, and simultaneously gives the correct 3-point correlation functions on the SYM side derived by perturbation theory, via our holographic mapping. As a byproduct, we also give of other approaches on the plane-wave holography, we would like to refer the reader to several review articles cited in [3]. 
some clarifications on the relation of our picture with other approaches which have been discussed in some of recent works for mapping the known versions of string field theories to gauge theory. We believe that our results not only resolve holography in the plane-wave limit, but also lay a foundation for understanding relation among different proposals and for investigating further extensions, on the basis of holography for correlation functions. In particular, we give a definite prediction for impurity non-preserving cases which have not been treated in the literature before.

The present paper is organized as follows. In section 2, we analyze the large $J$ limit directly on the usual diagrammatic rules ('Witten diagrams') for computing 3-point functions from the viewpoint of bulk theory. We demonstrate how our tunneling picture arises in the WKB approximation and establish the validity of this picture by comparing with exact computations. In section 3, we derive directly the effective action in the planewave limit from the bulk supergravity effective action given previously in ref. [11]. We then formulate the holographic relation which should be valid for (non BPS) stringy operators. In section 4, we construct the 'holographic' string field theory which is consistent with our effective action for supergravity sector. In section 5, we clarify the relation of our results with other approaches. In section 6, we confirm our general discussion by explicit examples. We conclude the paper in section 7 by giving further remarks. Two appendices $\mathrm{A}$ and $\mathrm{B}$ are devoted to some details of calculation and to a summary of the properties of string-interaction vertices of string field theory, respectively.

\section{The direct large $J$-limit of GKP-W relation}

Let us start from briefly recalling the standard perturbative computation of correlation functions from bulk supergravity theory. For simplicity, suppose that the bulk theory is effectively described by scalar fields $\phi_{i}(x)$ in the $\mathrm{AdS}_{5}$ background with action,

$$
S_{\phi}=\int d^{5} x \sqrt{g}\left[\sum_{i=1}^{3} \frac{1}{2}\left(\partial \phi_{i}\right)^{2}+\sum_{i=1}^{3} \frac{m_{i}^{2}}{2} \phi_{i}^{2}+g \phi_{1} \phi_{2} \phi_{3}\right], \quad m_{i}^{2}=J_{i}\left(J_{i}+4\right) / R^{2} .
$$

We assume the Euclidean AdS metric $\left(R^{4}=4 \pi g_{s} N\left(\alpha^{\prime}\right)^{2}\right)$,

$$
d s^{2}=\frac{R^{2}}{z^{2}}\left(d z^{2}+d \vec{x}^{2}\right)
$$

using the Poincaré coordinate. According to the GKP-W dictionary, a 3-point correlation function of three operators $\mathcal{O}_{i}\left(\vec{x}_{i}\right)$ (conformal dimension $=\Delta_{i}=J_{i}+\tilde{k}_{i}=k_{i}$ ) correspond- 
ing to the bulk fields $\phi_{i}$ is given, up to an overall normalization factor and to the lowest order with respect to the coupling constant $g$, as

$$
\left\langle\mathcal{O}_{1}\left(\vec{x}_{1}\right) \mathcal{O}_{2}\left(\vec{x}_{2}\right) \mathcal{O}_{3}\left(\vec{x}_{3}\right)\right\rangle=\int \frac{d^{4} \vec{x} d z}{z^{5}} K_{\Delta_{1}}\left(z, \vec{x} ; \vec{x}_{1}\right) K_{\Delta_{2}}\left(z, \vec{x} ; \vec{x}_{2}\right) K_{\Delta_{3}}\left(z, \vec{x} ; \vec{x}_{3}\right)
$$

where the bulk-to-boundary propagator

$$
K_{\Delta}(z, \vec{x} ; \vec{y})=\frac{\Gamma(\Delta)}{\pi^{2} \Gamma(\Delta-2)}\left(\frac{z}{z^{2}+(\vec{x}-\vec{y})^{2}}\right)^{\Delta}
$$

satisfies

$$
\left[z^{5} \frac{\partial}{\partial z}\left(z^{-3} \frac{\partial}{\partial z}\right)+z^{2} \frac{\partial^{2}}{\partial \vec{x}^{2}}-m^{2}\right] K_{\Delta}(z, \vec{x} ; \vec{y})=0, \quad \text { for } z>0
$$

and

$$
\lim _{z \rightarrow 0} z^{\Delta-4} K_{\Delta}(z, \vec{x} ; \vec{y})=\delta(\vec{x}-\vec{y}) .
$$

The PP-wave limit amounts to taking the limit where $J_{i}, R \rightarrow \infty$ with $J_{i} \alpha^{\prime} / R^{2}$ being kept fixed and $\tilde{k}_{i} \sim O(1)$. The angular momentum which comes from an $\mathrm{SO}(2)$ part of the $\mathrm{SO}(6)$ R-symmetry must be assumed to be conserved, say, $J_{1}=J_{2}+J_{3}$. Obviously, since $\Delta_{i} \sim J_{i} \rightarrow \infty$, the integral in the expression eq. (2.3) can be studied by saddle-point methods. ${ }^{\S}$ For our purpose it is useful to do a warm-up in the case of two-point functions. In what follows until stated otherwise explicitly, it is convenient to adopt the unit such that the $\mathrm{AdS}_{5}\left(S^{5}\right)$ radius to be one, $R=1$, since $R$ is the only length scale characterizing this system in the supergravity limit $\alpha^{\prime} \rightarrow 0$ with fixed $R$.

\subsection{Two-point functions}

Consider a 2-point function of the following form

$$
G_{2}\left(\vec{x}_{1}, \vec{x}_{2}\right) \equiv \int \frac{d^{4} \vec{x} d z}{z^{5}} K_{\Delta}\left(z, \vec{x} ; \vec{x}_{1}\right) K_{\Delta}\left(z, \vec{x} ; \vec{x}_{2}\right) z^{\epsilon}
$$

where $\epsilon \rightarrow 0+$ is a parameter for regularization. The saddle-point equations are

$$
\begin{aligned}
& \frac{\partial}{\partial z}\left(\ln \left[\frac{z}{z^{2}+\left(\vec{x}-\vec{x}_{1}\right)^{2}}\right]+\ln \left[\frac{z}{z^{2}+\left(\vec{x}-\vec{x}_{2}\right)^{2}}\right]\right)=0, \\
& \frac{\partial}{\partial \vec{x}}\left(\ln \left[\frac{z}{z^{2}+\left(\vec{x}-\vec{x}_{1}\right)^{2}}\right]+\ln \left[\frac{z}{z^{2}+\left(\vec{x}-\vec{x}_{2}\right)^{2}}\right]\right)=0 .
\end{aligned}
$$

$\S$ A preliminary discussion on the approach of the present paper has been given by one of the present authors in a talk at the Strings 2003 conference [12]. 
The general solution is

$$
\vec{x}_{0}=\frac{1}{2}\left(\vec{x}_{1}+\vec{x}_{2}\right)-\frac{1}{2}\left(\vec{x}_{1}-\vec{x}_{2}\right) \tanh \tau, \quad z_{0}=\frac{\left|\vec{x}_{1}-\vec{x}_{2}\right|}{2 \cosh \tau}
$$

with $\tau$ being an undermined integration constant. Thus the integral can be approximated as a one dimensional integral over the 'collective' coordinate $\tau$. In conformity with our previous works, the solution describes a tunneling process from one boundary point $\vec{x}_{1}$ at $\tau \sim-\infty$ to another boundary point $\vec{x}_{2}$ at $\tau \sim+\infty$. Thus we have naturally arrived at the same picture for the PP-wave holography as we have proposed in previous works.

Following the standard method of semi-classical path-integrals, the integration measure in (2.3) is replaced by

$$
\frac{d z d^{4} \vec{x}}{z^{5}} \Rightarrow d \tau d \tilde{z} d^{3} \overrightarrow{\tilde{x}}_{\perp} J(\tau)
$$

for the collective coordinate $\tau$ and the fluctuating coordinates $\tilde{z}, \overrightarrow{\tilde{x}}$, which are defined by the following shift of the bulk coordinates,

$$
z=z_{0}(\tau)+\tilde{z}, \quad \vec{x}=\vec{x}_{0}(\tau)+\overrightarrow{\tilde{x}}
$$

with the orthogonality constraint

$$
\frac{d z_{0}}{d \tau} \delta \tilde{z}+\frac{d \vec{x}_{0}}{d \tau} \cdot \delta \overrightarrow{\tilde{x}}=0
$$

The effective metric for the fluctuations is found to be, to the lowest nontrivial order in the fluctuations,

$$
\frac{1}{z^{2}}\left(\left(d z_{0}+d \delta \tilde{z}\right)^{2}+\left(d \vec{x}_{0}+d \overrightarrow{\delta \tilde{x}}\right)^{2}\right) \Rightarrow(d \tau)^{2}+\frac{4 \cosh ^{2} \tau}{\left|\vec{x}_{1}-\vec{x}_{2}\right|^{2}}\left(\cosh ^{2} \tau(d \delta \tilde{z})^{2}+\left(d \delta \overrightarrow{\tilde{x}}_{\perp}\right)^{2}\right)
$$

where we have used the solution for the constraint (2.13),

$$
\delta \overrightarrow{\tilde{x}}=-\vec{n} \sinh \tau \delta \tilde{z}+\delta \overrightarrow{\tilde{x}}_{\perp}, \quad \vec{n} \cdot \delta \overrightarrow{\tilde{x}}_{\perp}=0
$$

with $\vec{n}$ being the unit vector along the direction of the vector $\vec{x}_{1}-\vec{x}_{2}$, which connects two points $\vec{x}_{1}, \vec{x}_{2}$ on the boundary. Thus, the Jacobian is given as

$$
J(\tau)=\sqrt{\left(\frac{4 \cosh ^{2} \tau}{\left|\vec{x}_{1}-\vec{x}_{2}\right|^{2}}\right)^{4} \cosh ^{2} \tau}
$$

On the other hand, the effective second-order action for the saddle-point integral is

$$
S_{\text {eff }}^{(2)}=\frac{4 \Delta}{\left|\vec{x}_{1}-\vec{x}_{2}\right|^{2}}\left(\cosh ^{4} \tau \tilde{z}^{2}+\cosh ^{2} \tau\left(\overrightarrow{\tilde{x}}_{\perp}\right)^{2}\right)+\epsilon \text {-dependent factor. }
$$


The classical part of the action gives only a factor which is independent of the collective coordinate $\tau$ and has the correct dependence on the distance of boundary points, as can be checked by using

$$
K_{\Delta}\left(z_{0}(\tau), \vec{x}_{0}(\tau) ; \vec{x}_{1,2}\right)=\frac{\Gamma(\Delta)}{\pi^{2} \Gamma(\Delta-2)}\left|\vec{x}_{1}-\vec{x}_{2}\right|^{-\Delta} \mathrm{e}^{ \pm \Delta \tau}
$$

where the sign on the exponentials depends on the points $\vec{x}_{1}$ or $\vec{x}_{2}$, respectively. It is now evident that the Jacobian factor is canceled by the integrations over the fluctuating coordinates, up to a $\Delta$-dependent proportionality constant. Consequently, the two-point function in the large $\Delta$ limit is given simply by

$$
G_{2}\left(\vec{x}_{1}, \vec{x}_{2}\right) \Rightarrow \frac{\Delta^{2}}{\pi^{2}}\left|\vec{x}_{1}-\vec{x}_{2}\right|^{-2(\Delta-\epsilon)} \int_{-\infty}^{+\infty} d \tau(2 \cosh \tau)^{-\epsilon}
$$

where the $\epsilon$ dependent contributions come from the factor $z^{\epsilon}$ in the defining expression (2.7). Thus, in the limit $\epsilon \rightarrow 0+$, we reproduce the correct behavior for two-point correlators for conformal operators, up to the pole singularity

$$
\int_{-\infty}^{+\infty} d \tau(2 \cosh \tau)^{-\epsilon} \sim \frac{2}{\epsilon}
$$

We can compare this result with that of exact integration:

$$
G_{2}\left(\vec{x}_{1}, \vec{x}_{2}\right)=\left(\frac{\Gamma(\Delta)}{\pi^{2} \Gamma(\Delta-2)}\right)^{2} \times \frac{\pi^{2}}{2} \frac{\Gamma(\Delta-2) \Gamma(\epsilon / 2)^{2}}{\Gamma(\Delta) \Gamma(\epsilon)}\left|\vec{x}_{1}-\vec{x}_{2}\right|^{-2(\Delta-\epsilon)} \sim \frac{2 \Delta^{2}}{\pi^{2}} \frac{1}{\epsilon}\left|\vec{x}_{1}-\vec{x}_{2}\right|^{-2 \Delta}
$$

Here we have used the general formula for this type of integral [9],

$$
\begin{gathered}
\int_{0}^{\infty} d z \int d^{D} \vec{x} \frac{z^{a}}{\left(z^{2}+\left(\vec{x}-\vec{x}_{1}\right)^{2}\right)^{b}\left(z^{2}+\left(\vec{x}-\vec{x}_{2}\right)^{2}\right)^{c}}=\left|\vec{x}_{1}-\vec{x}_{2}\right|^{1+a+D-2 b-2 c} I(a, b, c, D), \\
I(a, b, c, D) \equiv \frac{\pi^{D / 2}}{2} \frac{\Gamma\left(\frac{a}{2}+\frac{1}{2}\right) \Gamma\left(b+c-\frac{D}{2}-\frac{a}{2}-\frac{1}{2}\right) \Gamma\left(\frac{1}{2}+\frac{a}{2}+\frac{D}{2}-b\right) \Gamma\left(\frac{1}{2}+\frac{a}{2}+\frac{D}{2}-c\right)}{\Gamma(b) \Gamma(c) \Gamma(1+a+D-b-c)} .
\end{gathered}
$$

In reality, for the effective theory described by (2.1), it is more appropriate to consider

$$
\int \frac{d^{4} \vec{x} d z}{z^{5-\epsilon}}\left[g^{\mu \nu}(z, \vec{x}) \partial_{\mu} K_{\Delta}\left(z, \vec{x} ; \vec{x}_{1}\right) \partial_{\nu} K_{\Delta}\left(z, \vec{x} ; \vec{x}_{2}\right)+m^{2} K_{\Delta}\left(z, \vec{x} ; \vec{x}_{1}\right) K_{\Delta}\left(z, \vec{x} ; \vec{x}_{2}\right)\right]
$$

than (2.7). It is easy to repeat the above calculation for this case. Only difference of the final result from the case of $G_{2}$ is the multiplication of $\left(m^{2}-\Delta^{2}\right) / m^{2} \sim-4 / \Delta$. 


\subsection{3-point functions}

Armed by this exercise, we now go back to the 3-point function (2.3). The saddle-point equations are

$$
\begin{gathered}
\sum_{i=1}^{3} \Delta_{i}\left(\frac{1}{z}-\frac{2 z}{z^{2}+\left(\vec{x}_{i}-\vec{x}\right)^{2}}\right)=0 \\
\sum_{i=1}^{3} \Delta_{i} \frac{\vec{x}_{i}-\vec{x}}{z^{2}+\left(\vec{x}_{i}-\vec{x}\right)^{2}}=0
\end{gathered}
$$

It is easy to convince oneself that, for generic configurations of three boundary points, there is no solution to these equations. However, if we take a limit where two of the boundary points, say, $\vec{x}_{2}$ and $\vec{x}_{3}$ approach sufficiently to one point $\vec{x}_{c}=\left(\vec{x}_{2}+\vec{x}_{3}\right) / 2$, the same trajectory connecting $\vec{x}_{1}$ and $\vec{x}_{c}$ as we have discussed in the previous subsection can be regarded as an approximate solution. Therefore, let us try to reduce the integral to the one along the following trajectory,

$$
\vec{x}_{0}(\tau)=\frac{1}{2}\left(\vec{x}_{1}+\vec{x}_{c}\right)-\frac{1}{2}\left(\vec{x}_{1}-\vec{x}_{c}\right) \tanh \tau, \quad z_{0}(\tau)=\frac{\left|\vec{x}_{1}-\vec{x}_{c}\right|}{2 \cosh \tau}
$$

with the fluctuations, $\tilde{\vec{x}}=\vec{x}-\vec{x}_{0}$ and $\tilde{z}=z-z_{0}$. For our purpose it is sufficient to evaluate the integral to the leading order in the short-distance limit,

$$
\vec{\delta} \equiv \vec{x}_{2}-\vec{x}_{c}=-\vec{x}_{3}+\vec{x}_{c} \rightarrow 0
$$

In order to avoid unnecessary complications, we assume that all three points $\vec{x}_{1}, \vec{x}_{2}, \vec{x}_{3}$ are along a single line on the boundary.

The effective action for this computation

$$
S_{e f f}=\sum_{i=1}^{3} \Delta_{i} \ln \frac{z^{2}+\left(\vec{x}_{i}-\vec{x}\right)^{2}}{z}
$$

is rewritten as

$$
\begin{gathered}
\frac{\Delta_{1}+\Delta_{2}+\Delta_{3}}{2}\left(\ln \frac{z^{2}+\left(\vec{x}_{1}-\vec{x}\right)^{2}}{z}+\frac{z^{2}+\left(\vec{x}_{c}-\vec{x}\right)^{2}}{z}\right) \\
+\frac{\Delta_{1}-\Delta_{2}-\Delta_{3}}{2}\left(\ln \frac{z^{2}+\left(\vec{x}_{1}-\vec{x}\right)^{2}}{z}-\ln \frac{z^{2}+\left(\vec{x}_{c}-\vec{x}\right)^{2}}{z}\right) \\
+\Delta_{2} \ln \left(1+\frac{2 \vec{\delta} \cdot\left(\vec{x}_{c}-\vec{x}\right)+\vec{\delta}^{2}}{z^{2}+\left(\vec{x}_{c}-\vec{x}\right)^{2}}\right)+\Delta_{3} \ln \left(1+\frac{-2 \vec{\delta} \cdot\left(\vec{x}_{c}-\vec{x}\right)+\vec{\delta}^{2}}{z^{2}+\left(\vec{x}_{c}-\vec{x}\right)^{2}}\right) .
\end{gathered}
$$


The first line of (2.28) can be treated in exactly the same way as for the 2-point case, by replacing $\Delta$ by $\left(\Delta_{1}+\Delta_{2}+\Delta_{3}\right) / 2\left(\sim J_{1} \rightarrow \infty\right)$. Next, since

$$
\Delta_{1}-\Delta_{2}-\Delta_{3} \sim O(1)
$$

the second line can be approximated by its value on the classical trajectory

$$
\frac{\Delta_{1}-\Delta_{2}-\Delta_{3}}{2}\left(\ln \frac{z_{0}^{2}+\left(\vec{x}_{1}-\vec{x}_{0}\right)^{2}}{z_{0}}-\ln \frac{z_{0}^{2}+\left(\vec{x}_{2}-\vec{x}_{0}\right)^{2}}{z_{0}}\right)=\left(\Delta_{1}-\Delta_{2}-\Delta_{3}\right) \tau
$$

The third line gives, to the zero-th order with respect to the fluctuations,

$$
-2\left(\Delta_{2}-\Delta_{3}\right) \frac{\delta}{\left|\vec{x}_{1}-\vec{x}_{c}\right|}-\left(\Delta_{2}+\Delta_{3}\right) \frac{\delta^{2}}{\left|\vec{x}_{1}-\vec{x}_{c}\right|^{2}}+\left(\Delta_{2}+\Delta_{3}\right) \frac{\delta^{2}}{\left|\vec{x}_{1}-\vec{x}_{c}\right|^{2}} \mathrm{e}^{2 \tau}+O\left(\delta^{3}\right) \text {. }
$$

Here we have kept the third term which is of second order in $\delta \rightarrow 0$, since it shows that for sufficiently large $\tau$ there is a natural cutoff for the range of the affine parameter for arbitrary small $\delta=|\vec{\delta}| \sim 0$. The other terms which are independent of $\tau$ can be ignored in the limit $\delta \rightarrow 0$.

As for the contribution of fluctuating coordinates in the third line, it is easy to convince ourselves that keeping only the first order term with respect both to the fluctuations and to the short-distance cutoff $\delta$ is sufficient for our purpose. The relevant terms are arranged as

$$
\begin{gathered}
-4\left(\Delta_{2}-\Delta_{3}\right)\left[\frac{\vec{\delta} \cdot\left(\vec{x}_{c}-\tilde{\vec{x}}\right)\left(\tilde{z} \cdot z_{0}-\tilde{\vec{x}} \cdot\left(\vec{x}_{c}-\vec{x}_{0}\right)\right)}{\left(z_{0}^{2}+\left(\vec{x}_{c}-\vec{x}_{0}\right)^{2}\right)^{2}}+\frac{1}{2} \frac{\vec{\delta} \cdot \tilde{\vec{x}}}{z_{0}^{2}+\left(\vec{x}_{c}-\vec{x}_{0}\right)^{2}}\right] \\
=\tilde{z} \frac{4 \delta\left(\Delta_{2}-\Delta_{3}\right)}{\left|\vec{x}_{1}-\vec{x}_{c}\right|^{2}} \mathrm{e}^{\tau} \cosh ^{2} \tau,
\end{gathered}
$$

which involves only the $\tilde{z}$-fluctuation. Combining this with the relevant part of the Gaussian factor coming from the first line, the integral with respect to $\tilde{z}$ is

$$
\begin{aligned}
& \int d \tilde{z}[\text { measure }] \exp \left[-\tilde{z}^{2} \frac{4 J_{1} \cosh ^{4} \tau}{\left|\vec{x}_{1}-\vec{x}_{2}\right|^{2}}-\tilde{z} \frac{4 \delta\left(\Delta_{2}-\Delta_{3}\right)}{\left|\vec{x}_{1}-\vec{x}_{c}\right|^{2}} \mathrm{e}^{\tau} \cosh ^{2} \tau\right] \\
= & \exp \left[\frac{\delta^{2}\left(\Delta_{2}-\Delta_{3}\right)^{2} \mathrm{e}^{2 \tau}}{J_{1}\left|\vec{x}_{1}-\vec{x}_{2}\right|^{2}}\right] \times \int d \tilde{z}[\text { measure }] \exp \left[-\tilde{z}^{2} \frac{4 J_{1} \cosh ^{4} \tau}{\left|\vec{x}_{1}-\vec{x}_{2}\right|^{2}}\right]
\end{aligned}
$$

to the present order of approximation. The prefactor here implies that, together with the contribution from the 3rd term of (2.31), the total factor which is responsible for the cutoff at large $\tau$ region is

$$
\exp \left[-\mathrm{e}^{2 \tau} \frac{\delta^{2}\left(\Delta_{2}+\Delta_{3}\right)}{\left|\vec{x}_{1}-\vec{x}_{2}\right|^{2}}+\mathrm{e}^{2 \tau} \frac{\left(\Delta_{2}-\Delta_{2}\right)^{2}}{J_{1}} \frac{\delta^{2}}{\left|\vec{x}_{1}-\vec{x}_{2}\right|^{2}}\right] \sim \exp \left[-4 \frac{\delta^{2}}{\left|\vec{x}_{1}-\vec{x}_{2}\right|^{2}} \frac{J_{2} J_{3}}{J_{1}} \mathrm{e}^{2 \tau}\right]
$$


Putting all of these results together and remembering that the Jacobian and the Gaussian integral cancel, the integral of the 3-point function now takes the form

$$
\frac{\pi^{2}}{J_{1}^{2}}\left|\vec{x}_{1}-\vec{x}_{c}\right|^{-\left(\Delta_{1}+\Delta_{2}+\Delta_{3}\right)} \int_{-\infty}^{+\infty} d \tau \mathrm{e}^{-\left(\Delta_{1}-\Delta_{2}-\Delta_{3}\right) \tau} \exp \left[-\frac{J_{2} J_{3}}{J_{1}} \frac{(2 \delta)^{2}}{\left|\vec{x}_{1}-\vec{x}_{c}\right|^{2}} \mathrm{e}^{2 \tau}\right]
$$

in the limit of large $J_{i}$ and small $\delta$. This expression shows that the precise form of the cutoff mentioned above is $\tau \lesssim \tau_{c}$ with

$$
e^{-\tau_{c}}=\sqrt{\frac{J_{2} J_{3}}{J_{1}}} \frac{2 \delta}{\left|\vec{x}_{1}-\vec{x}_{c}\right|} .
$$

Thus, the 3-point integral can be expressed by a Gamma function and leads to

$$
\frac{\pi^{2}}{J_{1}^{2}}\left|\vec{x}_{1}-\vec{x}_{c}\right|^{-2 \Delta_{1}}(2 \delta)^{-\left(\Delta_{2}+\Delta_{3}-\Delta_{1}\right)}\left(\frac{J_{2} J_{3}}{J_{1}}\right)^{-\left(\Delta_{2}+\Delta_{3}-\Delta_{1}\right) / 2} \frac{\Gamma\left(\frac{\Delta_{2}+\Delta_{3}-\Delta_{1}}{2}+1\right)}{-\Delta_{1}+\Delta_{2}+\Delta_{3}} \lambda .
$$

After fixing the convention for normalization suitably, this give the correct short-distance limit for the 3-point correlator, from which we can identify the CFT coefficient by equating the expression with

$$
\lim _{\vec{\delta} \rightarrow 0} \frac{C_{123}}{\left|\vec{x}_{1}-\vec{x}_{2}\right|^{2 \alpha_{3}}\left|\vec{x}_{2}-\vec{x}_{3}\right|^{2 \alpha_{1}}\left|\vec{x}_{3}-\vec{x}_{1}\right|^{2 \alpha_{2}}} \sim \frac{C_{123}}{\left|\vec{x}_{1}-\vec{x}_{c}\right|^{2 \Delta_{1}}|2 \vec{\delta}|^{2 \alpha_{1}}}
$$

where $\alpha_{1}=\left(\Delta_{2}+\Delta_{3}-\Delta_{1}\right) / 2$ etc. The precise normalization will be discussed in the next section.

Let us compare this result with the exact computation. The same integral formula cited before gives

$$
\begin{aligned}
& \int \frac{d^{4} \vec{x} d z}{z^{5}} \prod_{i=1}^{3}\left(\frac{z}{z^{2}+\left(\vec{x}-\vec{x}_{i}\right)^{2}}\right)^{\Delta_{i}}=\frac{c\left(\Delta_{1}, \Delta_{2}, \Delta_{3}\right)}{\left|\vec{x}_{1}-\vec{x}_{2}\right|^{\Delta_{1}+\Delta_{2}-\Delta_{3}}\left|\vec{x}_{2}-\vec{x}_{3}\right|^{\Delta_{2}+\Delta_{3}-\Delta_{1}}\left|\vec{x}_{3}-\vec{x}_{1}\right|^{\Delta_{3}+\Delta_{1}-\Delta_{2}}} \\
& c\left(\Delta_{1}, \Delta_{2}, \Delta_{3}\right)=\frac{\pi^{2}}{2} \frac{\Gamma\left(\frac{1}{2}\left(\Delta_{1}+\Delta_{2}-\Delta_{3}\right)\right) \Gamma\left(\frac{1}{2}\left(\Delta_{2}+\Delta_{3}-\Delta_{1}\right)\right) \Gamma\left(\frac{1}{2}\left(\Delta_{3}+\Delta_{1}-\Delta_{2}\right)\right)}{\Gamma\left(\Delta_{1}\right) \Gamma\left(\Delta_{2}\right) \Gamma\left(\Delta_{3}\right)} \\
& \times \Gamma\left(\frac{1}{2}\left(\Delta_{1}+\Delta_{2}+\Delta_{3}-4\right)\right) .
\end{aligned}
$$

By taking the large $J_{i}$ limit using the Stirling formula $\lim _{J \rightarrow \infty} \Gamma(J+\tilde{k}) \sim \sqrt{2 \pi} \exp ((J+$ $\left.\left.\tilde{k}-\frac{1}{2}\right) \ln J-J\right)$, we obtain

$$
\frac{\pi^{2}}{J_{1}^{2}}\left|\vec{x}_{1}-\vec{x}_{c}\right|^{-2 \Delta_{1}}(2 \delta)^{-\left(\Delta_{2}+\Delta_{3}-\Delta_{1}\right)}\left(\frac{J_{2} J_{3}}{J_{1}}\right)^{-\left(\Delta_{2}+\Delta_{3}-\Delta_{1}\right) / 2} \frac{\Gamma\left(\frac{\Delta_{2}+\Delta_{3}-\Delta_{1}}{2}+1\right)}{-\Delta_{1}+\Delta_{2}+\Delta_{3}},
$$

which exactly matches (2.37). 
Thus we have confirmed that the 3 -point correlators in the short distance limit $\vec{\delta} \rightarrow 0$ can be computed effectively as a process in the bulk occurring along a single tunneling trajectory connecting boundary to boundary, whose amplitude essentially takes the general form,

$$
\begin{gathered}
\frac{\epsilon^{\Delta_{1}-\Delta_{2}-\Delta_{3}}}{-\Delta_{1}+\Delta_{2}+\Delta_{3}} \tilde{g}, \\
\tilde{g} \sim g\left(\frac{J_{2} J_{3}}{J_{1}}\right)^{\frac{\Delta_{1}-\Delta_{2}-\Delta_{3}}{2}} \Gamma\left(\frac{-\Delta_{1}+\Delta_{2}+\Delta_{3}}{2}+1\right), \quad \epsilon=2 \delta,
\end{gathered}
$$

with a suitable normalization convention, apart from the usual spacetime dependent factor $\left|\vec{x}_{1}-\vec{x}_{c}\right|^{-2 \Delta_{1}}$.

The form (2.42) is consistent with what we have proposed as the general structure for 3-point correlators which is expected from the spacetime picture for holography in the PP-wave limit. The parameter $\epsilon \sim \mathrm{e}^{-\tau_{c}}$ is the cutoff related to the distance of the operators $\mathcal{O}_{2}\left(\vec{x}_{2}\right)$ and $\mathcal{O}_{3}\left(\vec{x}_{3}\right)$ at the boundary, as (2.36). In our first original discussion, the emergence of this particular form is originated from the formal integral $\int d \tau \exp \left[\left(\Delta_{1}-\right.\right.$ $\left.\left.\Delta_{2}-\Delta_{3}\right) \tau\right]$ which appears in perturbation theory along the tunneling trajectory. This is actually ill-defined as it stands. To justify the expression (2.42), we had to invoke a wave-packet picture which is rather subtle in the Euclidean tunneling as is alluded to in [4. According to the present argument, the ill-defined integral must be replaced by (2.35) which can be defined unambiguously by analytic continuation, due to the presence of the natural cutoff for large $\tau$ region. Intuitively, the large $\tau$ cutoff corresponds to the limitation of the picture using the single tunneling trajectory in discussing 3-point correlators for small but nonzero $\delta$. Note that if we take the limit $\delta \rightarrow 0$ naively inside the integral, this factor would have simply disappeared. In fact, the additional factor $\left(\frac{J_{2} J_{3}}{J_{1}}\right)^{\left(\Delta_{1}-\Delta_{2}-\Delta_{3}\right) / 2} \Gamma\left(\frac{-\Delta_{1}+\Delta_{2}+\Delta_{3}}{2}+1\right)$ was missing in the previous discussions, owing to this ambiguity.

It is known that the coupling constants of supergravity modes in the bulk vanish for the so-called 'extremal' case with $\Delta_{1}-\Delta_{2}-\Delta_{3}=0$. However, as is well known, the extremal correlation functions themselves do not vanish [10]. This remarkable fact corresponds to the presence of the singular denominator in (2.42). We propose that the holographic correspondence relating the bulk 3-point couplings and the CFT coefficients should obey the above general relation. Of course, the computation of this section is restricted to the supergravity approximation. Below, we will argue that this should generalize to non-BPS 
stringy modes too, with slight corrections. Actually, if we only consider a restricted set of the correlators in which the numbers of 'impurities' are conserved, as has been assumed in the literature, the correction factor can be ignored in the large $\mu$ limit to the leading order in making comparison with perturbative computation on the Yang-Mills side, since $\Delta_{1}-\Delta_{2}-\Delta_{3} \sim O\left(1 / \mu^{2}\right)$ for such cases.

\section{Effective action along the tunneling trajectory}

Since we have established that the computation of correlation functions (or CFT coefficients) can be reduced to the processes along a single tunneling trajectory connecting AdS boundary to AdS boundary, we can now proceed to derive an effective action along the trajectory directly from a more general effective action of supergravity in the bulk.

\subsection{Bulk effective action and the CFT coefficients}

We study the bulk effective action for general chiral primary operators consisting of the $\mathrm{SO}(6)$ scalar fields $\phi_{i}(x)$

$$
O^{I}=\kappa \mathcal{C}_{i_{1} i_{2} \cdots i_{k}}^{I} \operatorname{Tr}\left(\phi^{i_{1}} \phi^{i_{2}} \cdots \phi^{i_{k}}\right) .
$$

Here, $\mathcal{C}$ is a totally symmetric traceless tensor whose contraction is normalized as

$$
\left\langle\mathcal{C}^{I_{1}} \mathcal{C}^{I_{2}}\right\rangle=\mathcal{C}_{i_{1} i_{2} \cdots i_{k}}^{I_{1}} \mathcal{C}^{I_{2}, i_{1} i_{2} \cdots i_{k}}=\delta^{I_{1} I_{2}}
$$

and $\kappa=(2 \pi)^{k} /\left(g_{Y M}^{2} N\right)^{k / 2} \sqrt{k}$ is the normalization factor such that the 2-point functions takes the form $\left(\vec{x}_{12}=\vec{x}_{1}-\vec{x}_{2}\right)$,

$$
\left\langle O^{I_{1}}\left(\vec{x}_{1}\right) O^{I_{2}}\left(\vec{x}_{2}\right)\right\rangle=\frac{\delta^{I_{1} I_{2}}}{\left|\vec{x}_{12}\right|^{2 k}} .
$$

Since the BMN operators in the supergravity sector are essentially contained in this set of local operators, it should be possible to derive the effective action along the tunneling trajectory starting from an appropriate effective action for these operators. The BMN supergravity modes are a subset of (3.1), expressed by using the complex basis for the $\mathrm{SO}(2)$ directions $i=5,6$ with $Z=\frac{1}{\sqrt{2}}\left(\phi_{5}+i \phi_{6}\right)$ :

$$
O^{I}=\tilde{\kappa} \tilde{\mathcal{C}}_{i_{1} i_{2} \cdots i_{\tilde{k}}}^{I} \operatorname{Tr}\left(Z^{J} \phi^{i_{1}} \phi^{i_{2}} \cdots \phi^{i_{\tilde{k}}}+\text { permutations }\right), \quad k=J+\tilde{k}, \quad J \rightarrow \infty,
$$

where $\tilde{\mathcal{C}}_{i_{1} i_{2} \cdots i_{\tilde{k}}}^{I}$ are now completely symmetrized traceless $\mathrm{SO}(4)$ tensors with unit normalization (similarly as (3.2)) under contraction. The normalization constant is related to 
that of the original set as

$$
\tilde{\kappa}=\kappa\left(\begin{array}{c}
k \\
J
\end{array}\right)^{-1 / 2}
$$

Note that in the large $J$-limit the operators with 'vector' excitations $D_{i} Z$ are regarded essentially as the derivatives of (3.4), and hence are included in this set defined by (3.4) if we suitably take into account the variation of the boundary coordinates $\vec{x}$. In fact, as we will argue later on we have to take a special care in the interpretation of vector excitations.

The bulk effective action for the $\mathrm{SO}(6)$ operators has been derived in ref. [11]:

$$
S_{\text {bulk }}=\frac{4 N^{2}}{(2 \pi)^{5}} \int d^{5} x \sqrt{g}\left[\sum_{I} \frac{1}{2}\left(\nabla \phi_{I}\right)^{2}+\frac{1}{2} k(k-4) \phi_{I}^{2}-\frac{1}{3} \sum_{I_{1}, I_{2}, I_{3}} \frac{\mathcal{G}_{I_{1} I_{2} I_{3}}}{\sqrt{A_{I_{1}} A_{I_{2}} A_{I_{3}}}} \phi_{I_{1}} \phi_{I_{2}} \phi_{I_{3}}\right]
$$

where we used Euclidean metric and assumed a particular normalization for the scalar fields:

$$
A_{I}=2^{6-k} \pi^{3} \frac{k(k-1)}{(k+1)^{2}} .
$$

This is the effective action in the sense that it reproduces correlation functions through the Witten diagrams as studied in the previous section. Therefore, the freedom of field redefinition on the bulk side and correspondingly the choice of the basis of chiral operators on the boundary side are both fixed already. Remarkably, the set of bulk fields $\left\{\phi_{I}\right\}$ corresponding to the set $\left\{O^{I}\right\}$ are effectively treated as scalar fields propagating in the $\mathrm{AdS}_{5}$ background without derivative coupling. The derivative interactions are removed by making a particular field redefinition in the derivation of this action. ${ }^{\top}$ Actually, the effective action (3.6) was obtained from the equation of motion of IIB supergravity. It was argued that the result must be correct including the normalization of the CFT coefficients by relating them to the R-symmetry currents which have been known to be given exactly by the free gauge theory. We refer the reader to [11] and the references therein for more details on this effective action. Also for a summary on the non-renormalization properties of 2-and 3-point functions of chiral operators, see e.g. [13] and the references therein.

The CFT coefficients $C^{I_{1} I_{2} I_{3}}$ of the above operators defined by

$$
\left\langle O^{I_{1}}\left(\vec{x}_{1}\right) O^{I_{2}}\left(\vec{x}_{2}\right) O^{I_{3}}\left(\vec{x}_{3}\right)\right\rangle=\frac{C^{I_{1} I_{2} I_{3}}}{\left|\vec{x}_{12}\right|^{2 \alpha_{3}}\left|\vec{x}_{23}\right|^{2 \alpha_{1}}\left|\vec{x}_{31}\right|^{2 \alpha_{2}}}
$$

with

$$
\alpha_{1}=\frac{\Delta_{2}+\Delta_{3}-\Delta_{1}}{2}, \quad \text { etc }
$$

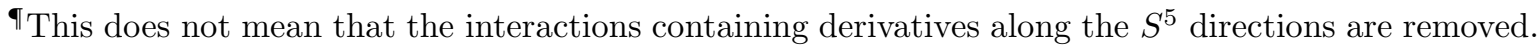


is related to the 3-point interaction in the effective action (3.6) by

$$
\begin{gathered}
C^{I_{1} I_{2} I_{3}}=\frac{\sqrt{k_{1} k_{2} k_{3}}}{N}\left\langle\mathcal{C}^{I_{1}} \mathcal{C}^{I_{2}} \mathcal{C}^{I_{3}}\right\rangle \\
\left\langle\mathcal{C}^{I_{1}} \mathcal{C}^{I_{2}} \mathcal{C}^{I_{3}}\right\rangle=\frac{\mathcal{G}_{I_{1} I_{2} I_{3}}}{a\left(k_{1}, k_{2}, k_{3}\right)} \frac{\left(k_{1}+1\right)\left(k_{2}+1\right)\left(k_{3}+1\right)}{2^{7} \Sigma\left((\Sigma / 2)^{2}-1\right)\left((\Sigma / 2)^{2}-4\right) \alpha_{1} \alpha_{2} \alpha_{3}} \\
\Sigma=k_{1}+k_{2}+k_{3} \\
a\left(k_{1}, k_{2}, k_{3}\right)=\frac{\pi^{3}}{\left(\frac{\Sigma}{2}+2\right) ! 2^{(\Sigma-2) / 2}} \frac{k_{1} ! k_{2} ! k_{3} !}{\alpha_{1} ! \alpha_{2} ! \alpha_{3} !}
\end{gathered}
$$

The first expression in this list is nothing but the result of free field computation of correlators on the Yang-Mills side, with $\left\langle\mathcal{C}^{I_{1}} \mathcal{C}^{I_{2}} \mathcal{C}^{I_{3}}\right\rangle$ being all the possible contractions among the $\mathrm{SO}(6)$ indices of $\mathcal{C}$-tensors. The second expression is its representation in terms of the bulk quantities, in which the factor $a\left(k_{1}, k_{2}, k_{3}\right)$ arises as the coefficient relating $\left\langle\mathcal{C}^{I_{1}} \mathcal{C}^{I_{2}} \mathcal{C}^{I_{3}}\right\rangle$ to the overlap integral of $S^{5}$ harmonics $Y^{I}$, which appears in reducing $10 \mathrm{D}$ theory to $5 \mathrm{D}$ effective theory on $\mathrm{AdS}_{5}$ backgrounds, as

$$
\int_{S^{5}} Y^{I_{1}} Y^{I_{2}} Y^{I_{3}}=a\left(k_{1}, k_{2}, k_{3}\right)\left\langle\mathcal{C}^{I_{1}} \mathcal{C}^{I_{2}} \mathcal{C}^{I_{3}}\right\rangle
$$

The definitions of the $S^{5}$ harmonics and their integrals are summarized in the Appendix A, to which we refer the reader for more details. The fact that the supergravity effective action is exactly matched to the free-field results on the gauge-theory side is interpreted as a consequence of non-renormalization properties of 3-point functions of chiral operators. This is quite remarkable since the supergravity limit $\alpha^{\prime} \rightarrow 0$ is nothing but a strongcoupling limit $g_{\mathrm{YM}}^{2} N=R^{4} /\left(\alpha^{\prime}\right)^{2} \rightarrow \infty$ on the gauge-theory side.

Before going to our main task of this section, let us here check that the relation between the above effective action and the CFT coefficients is consistent with the prediction of previous section. Taking the limit $J_{1}\left(=J_{2}+J_{3}\right), J_{2}, J_{3} \rightarrow \infty$, we find

$$
C^{I_{1} I_{2} I_{3}}=\frac{1}{N} \frac{2^{J_{1}+\frac{\tilde{\Sigma}}{2}-9}}{\pi^{3}} \frac{\sqrt{J_{1} J_{2} J_{3}}}{J_{1}^{2}}\left(\frac{J_{1}}{J_{2} J_{3}}\right)^{\alpha_{1}} \frac{\alpha_{1} !}{\alpha_{1}} \mathcal{G}_{I_{1} I_{2} I_{3}} .
$$

Apart from the normalization factor which is independent of $\alpha_{1}=\left(\Delta_{2}+\Delta_{3}-\Delta_{1}\right) / 2=$ $\left(\tilde{k}_{2}+\tilde{k}_{3}-\tilde{k}_{1}\right) / 2 \equiv \tilde{\alpha}_{1}$, this indeed coincides with the result obtained in the previous section, as it should. The difference of the normalization is owing to the fact that we have not yet fixed the precise normalization of two-point functions in the previous section. 
The CFT coefficients can be expressed in terms of the $\mathrm{SO}(4)$ basis (3.4) of the BMN operators, using the relation

$$
\left\langle\mathcal{C}^{\bar{I}_{1}} \mathcal{C}^{I_{2}} \mathcal{C}^{I_{3}}\right\rangle=\alpha_{1} !\left(\frac{J_{1}}{J_{2} J_{3}}\right)^{\tilde{\alpha}_{1}}\left(\frac{J_{2}}{J_{1}}\right)^{\tilde{k}_{2} / 2}\left(\frac{J_{3}}{J_{1}}\right)^{\tilde{k}_{3} / 2} \frac{\sqrt{\tilde{k}_{1} ! \tilde{k}_{2} ! \tilde{k}_{3} !}}{\tilde{\alpha}_{1} ! \tilde{\alpha}_{2} ! \tilde{\alpha}_{3} !}\left\langle\tilde{\mathcal{C}}^{\bar{I}_{1}} \tilde{\mathcal{C}}^{I_{2}} \tilde{\mathcal{C}}^{I_{3}}\right\rangle,
$$

which is valid in the large $J_{i}$ limit. For a derivation of this relation, see the Appendix A. Thus the 3-point coupling coefficient in the above effective action is

$$
\mathcal{G}_{\bar{I}_{1} I_{2} I_{3}}=\alpha_{1} \pi^{3} 2^{-J_{1}-\frac{\tilde{\Sigma}}{2}+9} J_{1}^{2}\left(\frac{J_{2}}{J_{1}}\right)^{\tilde{k}_{2} / 2}\left(\frac{J_{3}}{J_{1}}\right)^{\tilde{k}_{3} / 2} \frac{\sqrt{\tilde{k}_{1} ! \tilde{k}_{2} ! \tilde{k}_{3} !}}{\tilde{\alpha}_{1} ! \tilde{\alpha}_{2} ! \tilde{\alpha}_{3} !}\left\langle\tilde{\mathcal{C}}^{\bar{I}_{1}} \tilde{\mathcal{C}}^{I_{2}} \tilde{\mathcal{C}}^{I_{3}}\right\rangle
$$

in terms of the $\mathrm{SO}(4)$ contractions.

\subsection{Effective $0+1$ dimensional action}

With these preparations, we are now in the position of deriving the effective action along the tunneling trajectory starting from (3.6). Let us parametrize the coordinates near the trajectory as in the previous section,

$$
z=z_{0}(\tau)+\tilde{z}, \quad \vec{x}=\vec{x}_{0}(\tau)+\tilde{\vec{x}}
$$

with

$$
\tilde{\vec{x}}=-\vec{n} \tilde{z} \sinh \tau+\tilde{\vec{x}}_{\perp} .
$$

Since the fluctuations around the trajectory can be assumed to be of order $1 / \sqrt{J}$ from the discussion of the previous section, we derive the effective metric which is correct to the second order in the fluctuations. The result is

$$
d s^{2}=\left(1+\tilde{z}^{2}+\tilde{\vec{x}}_{\perp}^{2}\right) d \tau^{2}+d \tilde{z}^{2}+d \tilde{\vec{x}}_{\perp}^{2}+\text { higher order terms }
$$

after rescaling as

$$
\tilde{z} \rightarrow \frac{\left|\vec{x}_{1}-\vec{x}_{c}\right|}{2 \cosh ^{2} \tau} \tilde{z}, \quad \tilde{\vec{x}}_{\perp} \rightarrow \frac{\left|\vec{x}_{1}-\vec{x}_{c}\right|}{2 \cosh \tau} \tilde{\vec{x}}_{\perp}
$$

and then making the shift of the time coordinate,

$$
\tau \rightarrow \tau+\frac{\sinh \tau}{2 \cosh \tau}\left(\tilde{z}^{2}+\tilde{\vec{x}}_{\perp}^{2}\right)
$$

To avoid notational confusions, we denote the rescaled fluctuations $\left(\tilde{\vec{x}}_{\perp}, \tilde{z}\right)$ by a four vector $\vec{y}=\left(y_{1}, y_{2}, y_{3}, y_{4}\right)$. Thus the original fluctuating four-vector $\tilde{\vec{x}}$ in the bulk is now expressed as

$$
\left(\tilde{\vec{x}}_{\perp}, \vec{n} \cdot \tilde{\vec{x}}\right)=\frac{\left|\vec{x}_{1}-\vec{x}_{c}\right|}{2 \cosh \tilde{\tau}}\left(y_{1}, y_{2}, y_{3},-\frac{\sinh \tilde{\tau}}{\cosh \tilde{\tau}} y_{4}\right)
$$


with the time parameter being redefined as

$$
\tau=\tilde{\tau}+\frac{\sinh \tilde{\tau}}{2 \cosh \tilde{\tau}} \vec{y}^{2}
$$

The effective metric

$$
d s^{2}=\left(1+\vec{y}^{2}\right) d \tilde{\tau}^{2}+d \vec{y}^{2}
$$

has an $\mathrm{SO}(4)$ symmetry with respect to the rotation of $\vec{y}$. We also note that, since the order of magnitude of $\vec{y}$ is supposed to be constant in $\tau$ on the basis of this $\tau$-independent metric, (3.20) implies that the original fluctuating coordinates $\tilde{\vec{x}}$ decrease as $\mathrm{e}^{-|\tau|}$, when we approach the boundary $\tau \rightarrow \infty$.

Using this metric, the quadratic terms of the bulk effective action (3.6) are given, to the accuracy of second order in $\vec{y}$, as

$$
\frac{4 N^{2}}{(2 \pi)^{5}} \int d \tilde{\tau} d^{4} \vec{y}\left[\left(1-\frac{1}{2} y^{2}\right) \partial_{\tilde{\tau}} \overline{\phi_{I}} \partial_{\tilde{\tau}} \phi_{I}+\partial_{y} \overline{\phi_{I}} \partial_{y} \phi_{I}+\left(1+\frac{1}{2} y^{2}\right)(J+\tilde{k})(J+\tilde{k}-4) \overline{\phi_{I}} \phi_{I}\right]
$$

Note that we have changed the $\mathrm{SO}(6)$ index to the complex $\mathrm{SO}(2) \times \mathrm{SO}(4)$ index.

The assumption that the magnitude of the fluctuating coordinates is of order $1 / \sqrt{J}$ is justified in the language of the effective action as follows. Redefine the fields by

$$
\begin{aligned}
& \phi(\tau, \vec{y})=\mathrm{e}^{-J \tilde{\tau}} \phi_{0}^{(J)}(\vec{y}) \psi(\tau), \\
& \bar{\phi}(\tau, \vec{y})=\mathrm{e}^{+J \tilde{\tau}} \phi_{0}^{(J)}(\vec{y}) \bar{\psi}(\tau),
\end{aligned}
$$

with

$$
\phi_{0}^{(J)}(\vec{y})=(J / \pi)^{2} \exp \left[-\frac{1}{2} J \vec{y}^{2}\right]
$$

being the ground state wave function for the kinetic operator for the fluctuating coordinates,

$$
h=-\partial_{y}^{2}+J^{2} \vec{y}^{2} .
$$

The hermiticity condition for our Euclidean field theory is [4]

$$
\bar{\phi}(\tau, \vec{y})=\phi(-\tau, \vec{y})
$$

which requires the choice of signs in the definition (3.25) on the exponential. Here and in what follows, we rewrite $\tilde{\tau}$ and $\tilde{z}$ by $\tau$ and $z$ without tilde again for notational brevity. 
In terms of the reduced field $\psi(\tau), \bar{\psi}(\tau)$, the leading term of the free action is then of order $\mathrm{O}(J)$, taking the familiar nonrelativistic form in $0+1$ dimensions as

$$
\frac{4 N^{2}}{(2 \pi)^{5}} \int d \tau J\left[\bar{\psi} \partial_{\tau} \psi-\partial_{\tau} \bar{\psi} \psi+2 \tilde{k} \bar{\psi} \psi\right] .
$$

The order $O\left(J^{2}\right)$ terms of the form $J^{2} \bar{\psi} \psi$ are canceled, and the zero-point energy of the operator (3.27) is responsible for cancelling a part of the order $O(J)$ term in the last term in the lagrangian density. Note that the term $\left|\partial_{\tilde{\tau}} \psi\right|^{2}$ is of order one and hence can be ignored in the large $J$-limit. Absorbing the normalization factor for the field, the free Hamiltonian is seen to be $\tilde{k}$ which correctly reproduces the $O(1)$ part of the 'energy' $\Delta=J+\tilde{k}$.

By performing the above redefinition for the cubic interaction term and rescaling, $\psi \rightarrow \frac{(2 \pi)^{5 / 2}}{2 N} \frac{1}{\sqrt{2 J}} \psi$, to renormalize the quadratic terms in the standard form, the total effective action is

$$
\begin{gathered}
\int d \tau \sum_{I}\left[\frac{1}{2}\left(\bar{\psi}_{I} \partial_{\tau} \psi_{I}-\partial_{\tau} \bar{\psi}_{I} \psi_{I}\right)+\tilde{k}_{I} \bar{\psi}_{I} \psi_{I}\right]+\frac{1}{2} \int d \tau \sum_{I_{1}, I_{2}, I_{3}} \lambda_{\bar{I}_{1}, I_{2}, I_{3}}\left(\bar{\psi}_{I_{1}} \psi_{I_{2}} \psi_{I_{3}}+\text { h.c. }\right), \\
\lambda_{\bar{I}_{1}, I_{2}, I_{3}}=\frac{1}{\pi^{3} N} 2^{-8+J_{1}} \frac{2^{\tilde{\Sigma} / 2}}{J_{1}^{2}} \sqrt{J_{1} J_{2} J_{3}} \mathcal{G}_{\bar{I}_{1}, I_{2}, I_{3}},
\end{gathered}
$$

with $J_{1}=J_{2}+J_{3}$. Comparing this result with the known relation (3.15) connecting the CFT coefficients to the 3-point coupling constant, we confirm that the relation is again precisely the one discussed in (2.42) and (2.43). This establishes that the effective theory along the tunneling trajectory for the BMN operators with only scalar excitations is (3.30).

Now it is very natural to extend this action by including the higher excited states with respect to the Schrödinger operator (3.27). Since the fluctuating coordinates near the boundary $z_{0} \rightarrow 0(\tau \sim \pm \infty)$ are essentially the four vector $\vec{x}$ of the $4 \mathrm{D}$ base space up to an exponential factor $\left|\vec{x}_{1}-\vec{x}_{c}\right| \mathrm{e}^{-|\tau|}$, taking these excited states into account seems to correspond to the inclusion of the vector excitations $D_{i} Z(\bar{Z})$ of the BPS-BMN operators. Here we write down this extension and will give a precise discussion of this correspondence in the next section. The decomposition (3.24) is generalized to the infinite expansion over the complete set of excited states,

$$
\phi_{I}(\tau, \vec{y})=\frac{(2 \pi)^{5 / 2}}{2 N} \frac{1}{\sqrt{2 J}} \mathrm{e}^{-J \tau} \sum_{n} \phi_{n}^{(J)}(\vec{y}) \psi_{n}(\tau),
$$




$$
\bar{\phi}_{I}(\tau, \vec{y})=\frac{(2 \pi)^{5 / 2}}{2 N} \frac{1}{\sqrt{2 J}} \mathrm{e}^{+J \tau} \sum_{n} \phi_{n}^{(J)}(\vec{y}) \bar{\psi}_{n}(\tau)
$$

where

$$
\phi_{n}^{(J)}(\vec{y})=\prod_{i=1}^{4}\left(\frac{J}{\pi}\right)^{1 / 4} \frac{2^{-n_{i} / 2}}{\sqrt{n_{i} !}} H_{n_{i}}(\sqrt{J} \vec{y}) \mathrm{e}^{-J y^{2} / 2}
$$

are the normalized eigenfunctions. The kinetic term is then extended to

$$
\sum_{I, n}\left[\frac{1}{2}\left(\bar{\psi}_{I, n} \partial_{\tau} \psi_{I, n}-\partial_{\tau} \bar{\psi}_{I, n} \psi_{I, n}\right)+\left(\tilde{k}_{I}+\sum_{i=1}^{4} n_{i}\right) \bar{\psi}_{I, n} \psi_{I, n}\right]
$$

and similarly for the interaction term

$$
\frac{1}{2} \sum_{I_{1}, n^{(1)}, I_{2}, n^{(2)}, I_{3}, n^{(3)}} \frac{1}{\pi^{3} N} 2^{-8+J_{1}} \frac{2^{\tilde{\Sigma} / 2}}{J_{1}^{2}} \sqrt{J_{1} J_{2} J_{3}}\left(\mathcal{G}_{\bar{I}_{1}, I_{2}, I_{3}}^{n^{(1)}, n^{(2)}, n^{(3)}} \bar{\psi}_{I_{1}, n^{(1)}} \psi_{I_{2}, n^{(2)}} \psi_{I_{3}, n^{(3)}}+\text { h.c. }\right) .
$$

with

$$
\mathcal{G}_{\bar{I}_{1}, I_{2}, I_{3}}^{n^{(1)}, n^{(2)}}=\mathcal{G}_{\bar{I}_{1}, I_{2}, I_{3}} \frac{\pi J_{1}}{J_{2} J_{3}} \int d^{4} \vec{y} \phi_{n^{(1)}}^{\left(J_{1}\right)}(\vec{y}) \phi_{n^{(2)}}^{\left(J_{2}\right)}(\vec{y}) \phi_{n^{(3)}}^{\left(J_{3}\right)}(\vec{y})
$$

\subsection{Vector excitations}

The form of the effective action (3.36) and (3.37) including higher excited states with respect to the fluctuations $\vec{y}$ indicates that the vector excitations of BMN operators in the bulk can be treated in the same manner as the scalar excitations. On the boundary, BMN operators with vector excitations restricted to supergravity modes can be regarded essentially as derivatives of those without vector excitations; for sufficiently large $J$,

$$
\begin{gathered}
\tilde{C}_{i_{1} i_{2} \cdots i_{\tilde{k}}}^{I} K_{j_{1} j_{2} \cdots j_{\ell}}^{L} \partial_{j_{1}} \partial_{j_{2}} \cdots \partial_{j_{\ell}} \operatorname{Tr}\left(Z^{J} \phi^{i_{1}} \phi^{i_{2}} \cdots \phi^{i_{\tilde{k}}}+\text { permutations }\right) \\
\sim \tilde{C}_{i_{1} i_{2} \cdots i_{\tilde{k}}}^{I} K_{j_{1} j_{2} \cdots j_{\ell}}^{L} \operatorname{Tr}\left(Z^{J-\ell} D_{j_{1}} Z D_{j_{2}} Z \cdots D_{j_{\ell}} Z \phi^{i_{1}} \phi^{i_{2}} \cdots \phi^{i_{\tilde{k}}}+\text { permutations }\right)
\end{gathered}
$$

where $K_{j_{1} j_{2} \cdots j_{\ell}}^{L}$ is again a completely symmetric and traceless tensor $\|^{\|}$along the base-space directions on the 4D boundary, with the normalization condition of the same type as for $\tilde{C}^{I}$. The 'permutation' of the second line means a summation over all possibilities of different orderings of operators including $D_{j} Z$. We expect that the excitations with respect to $y_{j}$ components correspond to the action of $\partial_{j}$ on the boundary, as suggested in the original BMN proposal. However, we encounter two puzzles with this naive expectation.

\footnotetext{
"Strictly speaking, we should include the derivatives of the scalar impurity fields and also higher derivatives appropriately in the right-hand side of (3.38). In the present section, we do not treat the trace part for simplicity. They will be partly considered in section 5 after we construct the holographic string field theory.
} 
Firstly, we have stressed in subsection 3.2 that the fluctuations with respect to the original 4-coordinate $\vec{x}$ vanish exponentially $\mathrm{e}^{-|\tau|}$ as we approach the boundary. We then naively expect that the higher excited states do not affect the boundary theory. However, this is actually as it should be in accordance with our S-matrix picture [4] for the PP-wave holography. When an asymptotic state is an excited state, we have to supply an additional energy-factor $\mathrm{e}^{\ell|\tau|}$ to the wave function, by the definition of the S-matrix, which would just cancel the decreasing exponential associated with $\vec{y}$ fluctuations. In the case of scalar excitations discussed in section 2, the boundary condition (2.6) of the bulk-boundary propagator already takes this into account, as is visible in the boundary condition (2.6) .

Secondly, the effective $0+1$ dimensional action clearly demands that the 2 -point functions must satisfy an orthogonality condition, since the quadratic terms are diagonalized. To the contrary, the two-point functions obtained from the standard form $\frac{1}{\left|\vec{x}_{1}-\vec{x}_{2}\right|^{2 \Delta}}$ by acting derivative do not satisfy othogonality: for instance,

$$
\begin{gathered}
\partial_{1, j} \ln \left|\vec{x}_{1}-\vec{x}_{2}\right|=\frac{n_{j}}{\left|\vec{x}_{1}-\vec{x}_{2}\right|} \\
\partial_{1, j_{1}} \partial_{2, j_{2}} \ln \left|\vec{x}_{1}-\vec{x}_{2}\right|=-\frac{1}{\left|\vec{x}_{1}-\vec{x}_{2}\right|^{2}}\left(\delta_{j_{1} j_{2}}-2 n_{j_{1}} n_{j_{2}}\right)
\end{gathered}
$$

with $n_{j}=\left(x_{1}-x_{2}\right)_{j} /\left|\vec{x}_{1}-\vec{x}_{2}\right|$. The appearance of the tensor factor $\delta_{j_{1} j_{2}}-2 n_{j_{1}} n_{j_{2}} \equiv C_{j_{1} j_{2}}$ is consistent with the relation (3.20) of fluctuating coordinates with the vector $\vec{y}$, requiring that the directions of $\vec{y}$ along $\vec{n}$ are actually opposite at two asymptotic regions $\tau \rightarrow \mp \infty$, corresponding to $\vec{x}_{1}$ and $\vec{x}_{2}$, respectively, at the boundary. The tensor $C_{i j}$ is usually associated with the conformal inversion $\vec{x} \rightarrow \vec{x} /|x|^{2}$. In our case, this is an automatic consequence of the bulk-boundary correspondence. However, (3.39) evidently leads to an inconsistency with the orthogonality between scalar and vector excitations. In the literature (see e.g. [14]), a particular way out of this difficulty has been discussed without a definite physical picture for holography. The suggested procedure recovers orthogonality only after taking the limit $\left|\vec{x}_{1}-\vec{x}_{2}\right| \rightarrow \infty$. Since our picture for holography must be valid for any finite $\left|\vec{x}_{1}-\vec{x}_{2}\right|$, it is not satisfactory from our standpoint.

From our viewpoint of a direct correspondence between bulk and boundary as clarified up to this point, the origin of this apparent discrepancy lies in an important difference with respect to the $\mathrm{SO}(4)$ symmetry on both sides. For the bulk, we use the metric (3.22) which characterizes the geometry close to the tunneling trajectory traversing from a fixed boundary point to another fixed boundary point. In particular, the trajectory 
is asymptotically orthogonal to the boundary spacetime, and the fluctuations near the boundary have an $\mathrm{SO}(4)$ symmetry under global rotations of $\vec{y}$. The $\mathrm{SO}(4)$ rotations must be performed simultaneously at all points along the trajectory, and consequently at both ends on the boundary. It is important to recognize that this $\mathrm{SO}(4)$ symmetry cannot be identified with the $\mathrm{SO}(4)$ isometry of the AdS metric with respect to $\vec{x}$, as is indicated by the relation (3.20). Since the latter isometry should correspond to the $\mathrm{SO}(4)$ symmetry of the boundary theory, the rotations of $\vec{y}$ cannot be directly related to the usual $\mathrm{SO}(4)$ symmetry at the boundary.

For the boundary theory, we are using the flat Euclidean metric along the boundary space. The change of a distance caused by a small variation of points in the flat Euclidean metric is not of second order with respect to the variations of coordinates.

$$
\left(\vec{x}_{1}+\delta \vec{x}_{1}-\vec{x}_{2}-\delta \vec{x}_{2}\right)^{2}=\left|\vec{x}_{1}-\vec{x}_{2}\right|^{2}\left[1+2\left(n \cdot \frac{\delta \vec{x}_{1}-\delta \vec{x}_{2}}{\left|\vec{x}_{1}-\vec{x}_{2}\right|}\right)+\left(\frac{\left|\delta \vec{x}_{1}-\delta \vec{x}_{2}\right|}{\left|\vec{x}_{1}-\vec{x}_{2}\right|}\right)^{2}\right] .
$$

This is not $\mathrm{SO}(4)$ symmetric under the rotation of $\vec{y}$. Note that the $\mathrm{SO}(4)$ transformations of $\vec{y}$ must be done with fixed fiducial points, $\vec{x}_{1}$ and $\vec{x}_{2}$, in such a way that the vicinities around them are simultaneously rotated. Obviously, such an $\mathrm{SO}(4)$ symmetry cannot be identified with the usual global $\mathrm{SO}(4)$ isometry of the original AdS metric. One might wonder that this is then a self-contradiction. But that is not so. As we have emphasized, the magnitude of fluctuations around the trajectory vanishes exponentially as we approach the boundary, and hence there is no contradiction. This is also consistent with the fact that the tunneling trajectory is a classical solution under the restriction that the fluctuations satisfy the Dirichlet boundary condition at $z=0$. The Euclidean S-matrix, however, requires us to 'blow up' the vanishing fluctuations near the boundary at the vicinities of the fiducial points by multiplying the powers of $\mathrm{e}^{|\tau|}=\mathrm{e}^{T} \rightarrow \infty$ in such a way that the $\mathrm{SO}(4)$ symmetry with respect to $\vec{y}$ is kept. The process of blowing-up amounts to introducing a particular UV cutoff for the boundary theory.

Therefore, the naive identification of the vector excitations in the bulk with the derivations $D_{i} Z$ on the boundary is not precise. We have to check from the bulk point of view how the small variations near the boundary are treated by deriving two-point functions for them following the approach of section 2. Suppose that external lines are general vector states corresponding to the operators (3.38) with two traceless symmetric tensors 
$K^{L_{1}}$ and $K^{L_{2}}$. This amounts to extracting the parts proportional to

$$
K_{j_{1} j_{2} \cdots j_{1}}^{L_{1}} y^{j_{1}} y^{j_{2}} \cdots y^{j_{\ell_{1}}}, \quad K_{j_{1}^{\prime} j_{2}^{\prime} \cdots j_{\ell_{2}}^{\prime}}^{L_{2}} y^{j_{1}^{\prime}} y^{j_{2}^{\prime}} \cdots y^{j_{\ell_{2}}^{\prime}}
$$

from the bulk-boundary propagators in the integrand (2.7),

$$
\left(\frac{z}{z^{2}+\left(\vec{x}+\delta \vec{x}-\vec{x}_{1}-\delta \vec{x}_{1}\right)^{2}}\right)^{J+\tilde{k}}, \quad\left(\frac{z}{z^{2}+\left(\vec{x}+\delta \vec{x}-\vec{x}_{2}-\delta \vec{x}_{2}\right)^{2}}\right)^{J+\tilde{k}},
$$

respectively. They are given by

$$
\begin{aligned}
& 2^{\ell_{1}} J^{\ell_{1}} \frac{x^{j_{1}} x^{j_{2}} \cdots x^{\ell_{1}}}{z^{\ell_{1}}}\left(\frac{z}{z^{2}+\left(\vec{x}-\vec{x}_{1}\right)^{2}}\right)^{J+\tilde{k}+\ell_{1}}, \\
& 2^{\ell_{2}} J^{\ell_{2}} \frac{\bar{x}^{j^{\prime}} \bar{x}^{j_{2}^{\prime}} \cdots \bar{x}^{j_{\ell_{2}^{\prime}}}}{z^{\ell_{2}}}\left(\frac{z}{z^{2}+\left(\vec{x}-\vec{x}_{2}\right)^{2}}\right)^{J+\tilde{k}+\ell_{2}}
\end{aligned}
$$

respectively, in the large $J_{i}$-limit, where

$$
\bar{x}^{i}=\left(\delta^{i j}-2 n^{i} n^{j}\right) x^{j} .
$$

Note that the use of redefined $\bar{x}^{i}$ is required by the change of sign in the relation (3.20) between the fluctuating coordinates with $\vec{y}$ in the limit $\tau= \pm T(T \rightarrow \infty)$. Although the 4-th components actually give the powers of $|\tanh \tau|$ in converting from $\vec{x} / z$ (or $\overrightarrow{\vec{x}} / z$ ) to $\vec{y}$, they can be replaced by one in the leading singular part with respect to the regularization $\epsilon$. Then, the result of saddle-point integral is

$$
\delta^{L_{1} L_{2}} \frac{J^{2}}{\pi^{2}}(2 J)^{\ell_{1}} \ell_{1} !\left|\vec{x}_{1}-\vec{x}_{2}\right|^{-2\left(J+\tilde{k}+\ell_{1}\right)} \delta^{L_{1} L_{2}} \frac{2}{\epsilon} .
$$

The Kronecker $\delta^{L_{1} L_{2}}$ and the prefactor arises by the Gaussian integral

$$
(2 J)^{\ell_{1}+\ell_{2}} K_{j_{1} j_{2} \cdots \ell_{1}}^{L_{1}} K_{j_{1}^{\prime} j_{2}^{\prime} \cdots j_{\ell_{2}}^{\prime}}^{L_{2}} \frac{\int d y^{4} y^{j_{1}} y^{j_{2}} \cdots y^{j_{\ell_{1}}} y^{j_{1}^{\prime}} y^{j_{2}^{\prime}} \cdots y^{j_{\ell_{2}}^{\prime}} \mathrm{e}^{-J y^{2}}}{\int d y^{4} \mathrm{e}^{-J y^{2}}}=\delta^{L_{1} L_{2}} \ell_{1} !(2 J)^{\ell_{1}} .
$$

In terms of derivatives with respect to $\left(\vec{x}_{1}, \vec{x}_{2}\right)$, this result is expressed as

$$
K_{j_{1} j_{2} \cdots j_{\ell_{1}}}^{L_{1}} \partial_{1, j_{1}} \partial_{1, j_{2}} \cdots \partial_{1, j_{\ell_{1}}} K_{j_{1}^{\prime} j_{2}^{\prime} \cdots j_{\ell_{2}}^{\prime}}^{L_{2}} \bar{\partial}_{2, j_{1}^{\prime}} \bar{\partial}_{2, j_{2}^{\prime}} \cdots \bar{\partial}_{2, j_{\ell_{2}}^{\prime}} \frac{1}{\left|\vec{x}_{12}\right|_{g}^{2(J+\tilde{k})}}=\frac{(2 J)^{\ell} \ell_{1} !}{\left|\vec{x}_{1}-\vec{x}_{2}\right|^{2\left(J+\tilde{k}+\ell_{1}\right)}} \delta^{L_{1} L_{2}}
$$

with

$$
\bar{\partial}_{i}=\left(\delta_{i j}-2 n_{i} n_{j}\right) \partial_{j}
$$


being the derivatives with respect to $\bar{x}^{i}$. Here the subscript $g$ for the distance $\left|\vec{x}_{12}\right|$ indicates that the derivatives are computed by assuming that the small variations with respect to the boundary positions are defined by

$$
\left|\vec{x}_{12}\right|^{2} \rightarrow\left(\vec{x}_{1}-\vec{x}_{2}\right)^{2}\left(1-2 \frac{\delta \vec{x}_{1} \cdot \vec{\delta}_{2}}{\left|\vec{x}_{1}-\vec{x}_{2}\right|^{2}}\right)
$$

instead of the naive expression (3.41). Apart from the terms $\delta \vec{x}_{1}^{2}, \delta \vec{x}_{2}^{2}$ which do not contribute owing to the traceless condition for the tensors $K^{L_{1}}$ and $K^{L_{2}}$, this amounts to dropping all terms which violates the $\mathrm{SO}(4)$ symmetry in (3.41). Strictly speaking, we should have denoted the derivatives $\partial_{i}, \bar{\partial}_{i}$ by using different notations to indicate the situation. Effectively, our prescription is equivalent to dropping terms which violates the $\mathrm{SO}(4)$ symmetry, and is consistent with the prescriptions adopted in other approaches. Practically, the following rule is valid: First we consider only the directions orthogonal to $\vec{n}$ to obtain $\mathrm{SO}(3)$ symmetric answer. Then, we extend the results formally to $\mathrm{SO}(4)$ symmetric ones.

The variation indicated by (3.49) can be interpreted as the variation of the distances measured using the Plane-wave metric (3.22). The minimal distance defined by the distance functional

$$
\ell\left(\vec{y}_{1}, \vec{y}_{2} ; T\right)=\int d \tau \sqrt{1+\vec{y}^{2}+\left(\frac{d \vec{y}}{d \tau}\right)^{2}} \sim \int d \tau\left(1+\frac{1}{2}\left(\vec{y}^{2}+\left(\frac{d \vec{y}}{d \tau}\right)^{2}\right)\right.
$$

with the boundary condition

$$
\vec{y}(T)=y_{1}, \quad \vec{y}(-T)=y_{2}
$$

behaves as

$$
\begin{aligned}
\ell\left(\vec{y}_{1}, \vec{y}_{2} ; T\right) & =2 T+\frac{1}{2}\left(\vec{y}_{1}^{2}+\vec{y}_{2}^{2}\right) \tanh 2 T-\frac{\vec{y}_{1} \cdot \vec{y}_{2}}{\sinh 2 T} \\
& \sim 2 T+\frac{1}{2}\left(\vec{y}_{1}^{2}+\vec{y}_{2}^{2}\right)-2 \vec{y}_{1} \cdot \vec{y}_{2} \mathrm{e}^{-2 T}+O\left(\mathrm{e}^{-4 T}\right)
\end{aligned}
$$

Thus the transition amplitude is

$$
\mathrm{e}^{-(J+\tilde{k}) \ell\left(\vec{y}_{1}, \vec{y}_{2} ; T\right)} \sim \mathrm{e}^{-2(J+\tilde{k}) T}\left(1-2 \frac{\delta \vec{x}_{1} \cdot \overline{\delta \vec{x}_{2}}}{\left|\vec{x}_{1}-\vec{x}_{2}\right|^{2}}\right)^{J+\tilde{k}}
$$

with

$$
\delta \vec{x}_{1} \sim \frac{\mathrm{e}^{-T} \vec{y}_{1}}{\left|\vec{x}_{1}-\vec{x}_{2}\right|}, \quad \overline{\delta \vec{x}_{2}} \sim \frac{\mathrm{e}^{-T} \vec{y}_{2}}{\left|\vec{x}_{1}-\vec{x}_{2}\right|}
$$


which are required by the relation (3.20) for large $|\tau| \rightarrow T$. Note that we can ignore the term $\frac{1}{2}\left(\vec{y}_{1}^{2}+\vec{y}_{2}^{2}\right)$ in (3.52), which corresponds to the redefinition (3.21) and does not contribute owing to the traceless condition.

We can extend the above argument to 3-point correlators with vector excitations. By an obvious simplification of notations, we are lead to the integral

$$
\begin{gathered}
\frac{\left(\prod_{i=1}^{3}\left(2 J_{i}\right)^{\ell_{i}} K^{L_{i}}\right)}{\int d y^{4} \mathrm{e}^{-J_{1} y^{2}}} \int d y^{4} y^{j_{1}} y^{j_{2}} \cdots y^{j_{\ell_{1}}} y^{j_{1}^{\prime}} y^{j_{2}^{\prime}} \cdots y^{j_{\ell_{2}}^{\prime}} y^{j_{1}^{\prime \prime}} y^{j_{2}^{\prime \prime}} \cdots y^{j_{\ell_{3}^{\prime \prime}}} \mathrm{e}^{-J_{1} y^{2}} \\
\times\left(\text { scalar integral with } \Delta_{i} \rightarrow J_{i}+\tilde{k}_{i}+\ell_{i}\right) \\
=2^{\left(\ell_{1}+\ell_{2}+\ell_{3}\right) / 2} J_{2}^{\beta_{3}} J_{3}^{\beta_{2}} \frac{\ell_{1} ! \ell_{2} ! \ell_{3} !}{\beta_{1} ! \beta_{2} ! \beta_{3} !} \frac{\left(\alpha_{1}+\beta_{1}-1\right) !}{\left(\alpha_{1}-1\right) !}\left\langle K^{L_{1}} K^{L_{2}} K^{L_{3}}\right\rangle \\
\times \frac{C^{I_{1} I_{2} I_{3}}}{\left|\vec{x}_{1 c}\right|^{2\left(J_{1}+\tilde{k}_{1}+\ell_{1}\right)}|2 \delta|^{2\left(\alpha_{1}+\beta_{1}\right)}}
\end{gathered}
$$

where

$$
\beta_{1}=\left(\ell_{2}+\ell_{3}-\ell_{1}\right) / 2, \quad \text { etc. }
$$

Taking into account the normalization factor, this leads to the CFT coefficient

$$
\begin{gathered}
C^{I_{1} I_{2} I_{3}, L_{1} L_{2} L_{3}}=\left(\frac{J_{1}}{J_{2} J_{3}}\right)^{\beta_{1}}\left(\frac{J_{2}}{J_{1}}\right)^{\ell_{2} / 2}\left(\frac{J_{3}}{J_{1}}\right)^{\ell_{3} / 2} \\
\times \frac{\sqrt{\ell_{1} ! \ell_{2} ! \ell_{3} !}}{\beta_{1} ! \beta_{2} ! \beta_{3} !} \frac{\left(\alpha_{1}+\beta_{1}-1\right) !}{\left(\alpha_{1}-1\right) !} C^{I_{1} I_{2} I_{3}}\left\langle K^{L_{1}} K^{L_{2}} K^{L_{3}}\right\rangle
\end{gathered}
$$

From the viewpoint of boundary, this result can again be formulated as the consequence of the replacements,

$$
\begin{gathered}
\left|\vec{x}_{12}\right|^{2} \rightarrow\left(\vec{x}_{1}-\vec{x}_{0}\right)^{2}\left(1-2 \frac{\delta \vec{x}_{1} \cdot \overrightarrow{\delta \vec{x}}_{2}}{\left|\vec{x}_{1}-\vec{x}_{0}\right|^{2}}\right), \quad\left|\vec{x}_{13}\right|^{2} \rightarrow\left(\vec{x}_{1}-\vec{x}_{0}\right)^{2}\left(1-2 \frac{\delta \vec{x}_{1} \cdot \vec{\delta}_{3}}{\left|\vec{x}_{1}-\vec{x}_{0}\right|^{2}}\right) \\
|2 \delta|^{2} \rightarrow(2 \delta)^{2}\left(1-2 \frac{\left.\overline{\delta \vec{x}_{2}} \cdot{\overrightarrow{\delta \vec{x}_{3}}}_{|2 \delta|^{2}}\right)}{\mid}\right.
\end{gathered}
$$

in acting the derivation

$$
K_{j_{1} j_{2} \cdots j_{\ell_{1}}}^{L_{1}} \partial_{1, j_{1}} \partial_{1, j_{2}} \cdots \partial_{1, j_{\ell_{1}}} K_{j_{1}^{\prime} j_{2}^{\prime} \cdots j_{\ell_{2}}^{\prime}}^{L_{2}} \bar{\partial}_{2, j_{1}^{\prime}} \bar{\partial}_{2, j_{2}^{\prime}} \cdots \bar{\partial}_{2, j_{\ell_{2}}^{\prime}} K_{j_{1} " j_{2}^{\prime} \cdots j^{\prime \prime} \ell_{3}}^{L_{3}} \bar{\partial}_{3, j_{1}} \bar{\partial}_{3, j_{3}} \cdots \bar{\partial}_{3, j^{\prime \prime} \ell_{3}}
$$

directly to the general form (3.8) of the 3-point correlator without vector excitations. The prescriptions (3.58) are equivalent to (3.49) to the leading order in $\delta$, while (3.59) is necessary to be consistent with the $\mathrm{SO}(4)$ symmetry. 
Combining with the CFT coefficient of pure scalars, we obtain

$$
\begin{aligned}
& C^{I_{1} I_{2} I_{3}, L_{1} L_{2} L_{3}}=\frac{1}{N} \frac{2^{J_{1}+\frac{\tilde{\Sigma}}{2}-9}}{\pi^{3}} \frac{\sqrt{J_{1} J_{2} J_{3}}}{J_{1}^{2}}\left(\frac{J_{1}}{J_{2} J_{3}}\right)^{\alpha_{1}+\beta_{1}} \frac{\left(\alpha_{1}+\beta_{1}\right) !}{\alpha_{1}+\beta_{1}} \mathcal{G}_{I_{1} I_{2} I_{3}} \\
& \times\left(\frac{J_{2}}{J_{1}}\right)^{\ell_{2} / 2}\left(\frac{J_{3}}{J_{1}}\right)^{\ell_{3} / 2} \frac{\sqrt{\ell_{1} ! \ell_{2} ! \ell_{3} !}}{\beta_{1} ! \beta_{2} ! \beta_{3} !}\left\langle K^{L_{1}} K^{L_{2}} K^{L_{3}}\right\rangle
\end{aligned}
$$

in terms of the pure-scalar 3-point coupling, or using the expression for the scalar vertex (3.17)

$$
\begin{gathered}
C^{\bar{I}_{1} I_{2} I_{3}, L_{1} L_{2} L_{3}}=\frac{1}{N} \frac{\sqrt{J_{1} J_{2} J_{3}}}{J_{1}^{2}}\left(\frac{J_{1}}{J_{2} J_{3}}\right)^{\alpha_{1}+\beta_{1}}\left(\alpha_{1}+\beta_{1}\right) ! \frac{\alpha_{1}}{\alpha_{1}+\beta_{1}} \\
\times\left(\frac{J_{2}}{J_{1}}\right)^{\left(\tilde{k}_{2}+\ell_{2}\right) / 2}\left(\frac{J_{3}}{J_{1}}\right)^{\left(\tilde{k}_{3}+\ell_{3}\right) / 2} \frac{\sqrt{\tilde{k}_{1} ! \tilde{k}_{2} ! \tilde{k}_{3} !}}{\tilde{\alpha}_{1} ! \tilde{\alpha}_{2} ! \tilde{\alpha}_{3} !}\left\langle\tilde{\mathcal{C}}^{\bar{I}_{1}} \tilde{\mathcal{C}}^{I_{2}} \tilde{\mathcal{C}}^{I_{3}}\right\rangle \frac{\sqrt{\ell_{1} ! \ell_{2} ! \ell_{3} !}}{\beta_{1} ! \beta_{2} ! \beta_{3} !}\left\langle K^{L_{1}} K^{L_{2}} K^{L_{3}}\right\rangle
\end{gathered}
$$

in terms of the $\mathrm{SO}(4)$ tensors. On the other hand, the effective interaction (3.37) including the vector excitations shows that the 3-point coupling for the external lines in consideration is given by replacing the coefficient $\mathcal{G}_{I_{1} I_{2} I_{3}}$ for purely scalar excitations as

$$
\mathcal{G}_{I_{1} I_{2} I_{3}}^{L_{1} L_{2} L_{3}}=\mathcal{G}_{I_{1} I_{2} I_{3}}\left(\frac{J_{2}}{J_{1}}\right)^{\ell_{2} / 2}\left(\frac{J_{3}}{J_{1}}\right)^{\ell_{3} / 2} \frac{\sqrt{\ell_{1} ! \ell_{2} ! \ell_{3} !}}{\beta_{1} ! \beta_{2} ! \beta_{3} !}\left\langle K^{L_{1}} K^{L_{2}} K^{L_{3}}\right\rangle .
$$

Comparing this with (3.60) taking account the normalization factor in the 3-point interaction term of the effective action, we can conclude that the general form, as derived in section 2 for the holographic correspondence of scalar supergravity excitations, extends to a more general case including the vector excitations,

$$
\begin{gathered}
C_{I_{1} I_{2} I_{3}}^{L_{1} L_{2} L_{3}}=\frac{\tilde{\lambda}_{I_{1} I_{2} I_{3}}^{L_{1} L_{2} L_{3}}}{\Delta_{2}+\Delta_{3}-\Delta_{1}} \\
\tilde{\lambda}_{I_{1} L_{2} I_{3}}^{L_{1} L_{2} L_{3}}=\left(\frac{J_{2} J_{3}}{J_{1}}\right)^{\left(\Delta_{1}-\Delta_{2}-\Delta_{3}\right) / 2} \Gamma\left(\frac{\Delta_{2}+\Delta_{3}-\Delta_{1}}{2}+1\right) \lambda_{I_{1} I_{2} I_{3}}^{L_{1} L_{2} L_{3}}
\end{gathered}
$$

with $\Delta_{i}=k_{i}+\ell_{i}=J_{i}+\tilde{k}_{i}+\ell_{i}$.

Supersymmetry demands that this should extend further to fermionic excitations. Since we treat the full supersymmetric string field theory later, we do not discuss the supersymmetrization of our arguments explicitly. We only mention that to discuss orthogonality including fermionic excitations, the spinor equivalent to the inversion tensor $\delta_{j_{1} j_{2}}-2 n_{j_{1}} n_{J_{2}}$ transforming spinor indices between two boundary points is the $\mathrm{SO}(4)$ gamma matrix $i \gamma^{i} n^{i}$. In computing correlation functions, we have to extract $\mathrm{SO}(4)$ invariant pieces in similar manner as for bosonic excitations by taking into account the transformation of spinor indices between two boundary points. This is effectively equivalent with the procedures adopted in other works. 


\subsection{Operator representation and the $\alpha^{\prime}$ corrections}

On the basis of our results, we are now prepared to proceed to the construction of a full 'holographic' string field theory. Before that, it is convenient to first rewrite the foregoing results using oscillator representation. By substituting the expression for the general 3 -point coupling, the effective action is written as

$$
\begin{gathered}
S_{\text {eff }}=S_{2}+S_{3} \\
S_{2}=\sum_{I, L} \int d \tau\left[\frac{1}{2}\left(\bar{\psi}_{I, L} \partial_{\tau} \psi_{I, L}-\partial_{\tau} \bar{\psi}_{I, L} \psi_{I, L}\right)+\frac{1}{R}\left(\tilde{k}_{I}+\ell_{L}\right) \bar{\psi}_{I, L} \psi_{I, L}\right], \\
S_{3}=\frac{1}{N R} \int d \tau \sum_{I_{1}, I_{2}, I_{3}} \alpha_{1} \sqrt{J_{1} J_{2} J_{3}} \bar{\psi}_{I_{1}, L_{1}} \psi_{I_{2}, L_{2}} \psi_{I_{3}, L_{3}}\left(\frac{J_{2}}{J_{1}}\right)^{\left(\tilde{k}_{2}+\ell_{2}\right) / 2}\left(\frac{J_{3}}{J_{1}}\right)^{\left(\tilde{k}_{3}+\ell_{3}\right) / 2} \\
\times \frac{\sqrt{\tilde{k}_{1} ! \tilde{k}_{2} ! \tilde{k}_{3}}}{\tilde{\alpha}_{1} ! \tilde{\alpha}_{2} ! \tilde{\alpha}_{3} !} \frac{\sqrt{\ell_{1} ! \ell_{2} ! \ell_{3} !}}{\beta_{1} ! \beta_{2} ! \beta_{3} !}\left\langle\tilde{\mathcal{C}}^{\bar{I}_{1}} \tilde{\mathcal{C}}^{I_{2}} \tilde{\mathcal{C}}^{I_{3}}\right\rangle\left\langle K^{L_{1}} K^{L_{2}} K^{L_{3}}\right\rangle+h . c .,
\end{gathered}
$$

where we have recovered the length dimension. The integrals over the angular momentum $J$ should be understood implicitly. We introduce the bra-ket notation for the field by using the 8 dimensional oscillator algebra of $a_{i}, a_{i}^{\dagger} ; a_{i}|0\rangle=0,\left[a_{i}, a_{j}^{\dagger}\right]=\delta_{i j}(i=1,2, \ldots, 8)$ where the first 4 directions correspond to the $\mathrm{SO}(4)$ indices of the vector directions $\vec{y}$, and the remaining 4 directions $(i=5, \ldots, 8)$ represents the $\mathrm{SO}(4)$ indices of the scalar excitations.

$$
\begin{gathered}
\left\{\psi_{I}(\tau)\right\} \rightarrow|\psi(\tau)\rangle=\sum_{I, L} \psi_{I, L}(\tau)|I, L\rangle \\
|I, L\rangle=\tilde{C}_{i_{1} i_{2} \cdots i_{\tilde{k}}}^{I} K_{j_{1} j_{2} \cdots j_{\ell}}^{L} \frac{1}{\sqrt{\tilde{k} ! \ell !}} a_{i_{1}}^{\dagger} a_{i_{2}}^{\dagger} \cdots a_{i_{\tilde{k}}}^{\dagger} a_{j_{1}}^{\dagger} a_{j_{2}}^{\dagger} \cdots a_{j_{\ell}}^{\dagger}|0\rangle .
\end{gathered}
$$

The free term of the action is then

$$
S_{2}=\int d \tau\left[\frac{1}{2}\left(\left\langle\bar{\psi}\left|\partial_{\tau}\right| \psi\right\rangle-\frac{1}{2}\left(\partial_{\tau}\langle\bar{\psi}|\right)|\psi\rangle+\left\langle\bar{\psi}\left|h_{s v}\right| \psi\right\rangle\right]\right.
$$

with

$$
h_{s v}=h_{s}+h_{v}, \quad h_{s}=\frac{1}{R} \sum_{i=1}^{4} a_{i}^{\dagger} a_{i}, \quad h_{v}=\frac{1}{R} \sum_{j=5}^{8} a_{j}^{\dagger} a_{j} .
$$

The parameters familiar in string field theory literature are $R^{4} / J_{1}^{2}\left(\alpha^{\prime}\right)^{2}=\lambda^{\prime}=1 /\left(\mu p^{+} \alpha^{\prime}\right)^{2}=$ $g_{Y M}^{2} N / J_{1}^{2}, g_{2}=J_{1}^{2} / N$. In terms of the string-length parameter, $\alpha_{(r)}=\alpha^{\prime} p_{r}^{+}, \mu\left|\alpha_{(r)}\right|=$ $\alpha^{\prime} J_{r} / R^{2}$. Note that $\mu \sim 1 / R$ since $\left|p_{r}^{+}\right| \sim J_{r} / R$ and also that $\alpha_{(1)}<0, \alpha_{(2)}>0, \alpha_{(3)}>0$ in the present convention. The supergravity limit corresponds to the limit $\alpha^{\prime} \rightarrow 0$ with fixed $R=\left(g_{\mathrm{YM}}^{2} N\right)^{1 / 4} \alpha^{\prime}$. 
The interaction term is expressed in terms of the overlap state defined by

$$
\left|v_{0}\right\rangle=\exp \left[-\frac{1}{2} \sum_{r, s=1}^{3}\left(\sum_{i=1}^{4} a_{i(r)}^{\dagger} n_{00}^{r s} a_{i(s)}^{\dagger}+\sum_{j=5}^{8} a_{j(r)}^{\dagger} n_{00}^{r s} a_{j(s)}^{\dagger}\right)\right]|0\rangle
$$

with the supergravity part of the familiar Neumann functions in the zero-slop limit $\alpha^{\prime} \rightarrow 0$.

$$
n_{00}^{r s}=\delta^{r s}-\sqrt{\frac{J_{r} J_{s}}{J_{1}^{2}}}, \quad n_{00}^{r 1}=n_{00}^{1 r}=-\sqrt{\frac{J_{r}}{J_{1}}} \quad \text { for } \quad r, s=2,3 \quad \text { and } \quad n_{00}^{11}=0
$$

The unusual minus sign on the exponent is owing to our phase convention for the creationannihilation operators. As is well known, the overlap state is the ground-state solution for the continuity conditions satisfying

$$
\sum_{r=1}^{3} p_{(r)}\left|v_{0}\right\rangle=0, \quad\left(x_{(3)}-\frac{J_{2}}{J_{1}} x_{(2)}-\frac{J_{3}}{J_{1}} x_{(3)}\right)\left|v_{0}\right\rangle=0
$$

where

$$
x_{(r)}=\frac{R}{\sqrt{2 J_{r}}}\left(a_{(r)}+a_{(r)}^{\dagger}\right), \quad p_{(r)}=-i \sqrt{\frac{J_{r}}{2 R^{2}}}\left(a_{(r)}-a_{(r)}^{\dagger}\right)
$$

for all 8 directions. The 'ground state' means that it also satisfy the locality condition of the form

$$
\left(x_{(1)}-x_{(2)}\right)\left|v_{0}\right\rangle=\left(x_{(1)}-x_{(3)}\right)\left|v_{0}\right\rangle=0 .
$$

We find that

$$
\begin{gathered}
{ }_{(1)}\left\langle\bar{I}_{1},\left.L_{1}\right|_{(2)}\left\langle I_{2},\left.L_{2}\right|_{(3)}\left\langle I_{3}, L_{3}|| v_{0}\right\rangle=\left(\frac{J_{2}}{J_{1}}\right)^{\frac{\left(\tilde{k}_{2}+\ell_{2}\right)}{2}}\left(\frac{J_{3}}{J_{1}}\right)^{\frac{\left(\tilde{k}_{3}+\ell_{3}\right)}{2}}\right.\right. \\
\times \frac{\sqrt{\tilde{k}_{1} ! \tilde{k}_{2} ! \tilde{k}_{3} !}}{\tilde{\alpha}_{1} ! \tilde{\alpha}_{2} ! \tilde{\alpha}_{3} !}\left\langle\tilde{C}^{I_{1}} \tilde{C}^{I_{2}} \tilde{C}^{I_{3}}\right\rangle \frac{\sqrt{\ell_{1} ! \ell_{2} ! \ell_{3} !}}{\beta_{1} ! \beta_{2} ! \beta_{3} !}\left\langle K^{L_{1}} K^{L_{2}} K^{L_{3}}\right\rangle .
\end{gathered}
$$

This shows that the 3 -point interaction part of the effective action takes the following simple form

$$
S_{3}=\frac{1}{2 N} \int d \tau_{(1)}\left\langle\overline { \psi } | _ { ( 2 ) } \left\langle\left.\psi\right|_{(3)}\left\langle\psi\left|\sqrt{J_{1} J_{2} J_{3}}\left(h_{s}^{(2)}+h_{s}^{(3)}-h_{s}^{(1)}\right)\right| v_{0}\right\rangle+\right.\right.\text { h.c. . }
$$

The integral with respect to the angular momentum under the conservation condition $J_{1}=$ $J_{2}+J_{3}$ should be understood implicitly as before. The most important characteristic of this expression is that the so-called 'prefactor' involves only the energies of scalar excitations. As we have stressed in previous works, the holographic relation of our type does not necessarily demand that the prefactor itself is the difference of the free Hamiltonian $h_{s v}^{(2)}+$ 
$h_{s v}^{(3)}-h_{s v}^{(1)}$. As a consequence of this remarkable property, the 3-point vertices vanish whenever the scalar energies are preserved. This implies that, except for special cases when the vector energies are simultaneously preserved, the 3-point correlators vanish, as has been explicitly exhibited in the expression of the CFT coefficient (3.61). Since we have constraints $\tilde{k}_{1} \leq \tilde{k}_{2}+\tilde{k}_{3}$ and $\ell_{1} \leq \ell_{2}+\ell_{3}$, the conservation of both scalar and vector energies is possible only when the total energies are conserved, namely, when $\Delta_{1}=\Delta_{2}+\Delta_{3}$. Note that in this extremal cases we have finite results for the 3-point correlators, owing to the vanishing denominator in our holographic relation.

Of course, the above action for supergravity modes is valid in the limit $\alpha^{\prime} \rightarrow 0$ with fixed $R$. The limit $\alpha^{\prime} \rightarrow 0$ corresponds to the imposition of the locality conditions (3.76) for the overlap state. However, when we consider a finite $\alpha^{\prime}$, the latter conditions cannot be preserved, as it should be for general string states which have nonzero extension. Then only the more general continuity conditions (3.74) are satisfied. Correspondingly, the Neumann functions $n_{00}^{r s}$ for the zero modes must be replaced by $N_{00}^{r s}$, defined by $N_{00}^{r s}=f n_{00}^{r s}=f\left(\delta^{r s}-\sqrt{\frac{J_{r} J_{s}}{J_{1}^{2}}}\right), \quad N_{00}^{r 1}=n_{00}^{r 1}=-\sqrt{\frac{J_{r}}{J_{1}}} \quad$ for $\quad r, s=2,3 \quad$ and $\quad N_{00}^{11}=0$

where $f=1-4 \mu \alpha_{(1)} \alpha_{(2)} \alpha_{(3)} K$ is a nontrivial function of $\mu \alpha_{(r)}(r=1,2,3)$. For the definition of the quantity $K$, we refer the reader to the Appendix B. In the supergravity limit $\alpha^{\prime} \rightarrow 0$, keeping $R$ and $J_{i}$ fixed, we have $f \rightarrow 1$. In the opposite large $\mu$ limit, it is known that

$$
f \rightarrow \frac{\left|\alpha_{(1)}\right|}{4 \pi \mu \alpha_{(2)} \alpha_{(3)}}=\frac{R^{2} J_{1}}{4 \pi \alpha^{\prime} J_{2} J_{3}} .
$$

We denote the overlap state with this modification for finite $\alpha^{\prime}$ by $\left|V_{0}\right\rangle$.

$$
\left|V_{0}\right\rangle=\exp \left[-\frac{1}{2} \sum_{r, s=1}^{3}\left(\sum_{i=1}^{4} a_{i(r)}^{\dagger} N_{00}^{r s} a_{i(s)}^{\dagger}+\sum_{j=5}^{8} a_{j(r)}^{\dagger} N_{00}^{r s} a_{j(s)}^{\dagger}\right)\right]|0\rangle
$$

The use of the $\alpha^{\prime}$-corrected 3-point vertex which is given by the same form as (3.78) but $\left|v_{0}\right\rangle$ being replaced by $\left|V_{0}\right\rangle$ leads to a correction factor $f^{\alpha_{1}}$ for the matrix elements, multiplying the original local form.

On the other hand, the normalization of the CFT coefficients for the BPS operators used in the foregoing subsections is believed to be exact to all orders in the gauge coupling $\lambda=g_{\mathrm{YM}}^{2} N$, because of the non-renormalization property of 3-point functions of chiral operators. Indeed, as we stressed before, the CFT coefficients are nothing but the results 
from the free field theory, which corresponds to the large $\mu$ limit. This implies that for nonzero $\alpha^{\prime}$, the rule of mapping between the matrix elements of the bulk 3-point vertex and the CFT coefficients must be extended as a natural ansatz to

$$
\begin{gathered}
C_{I_{1} I_{2} I_{3}}^{L_{1} L_{2} L_{3}}=\frac{\tilde{\lambda}_{I_{1} L_{2} I_{3}}^{L_{1} L_{2} L_{3}}}{\Delta_{2}+\Delta_{3}-\Delta_{1}}, \\
\tilde{\lambda}_{I_{1} L_{2} I_{3}}^{L_{2} L_{2} L_{3}}=\left(f \frac{J_{2} J_{3}}{J_{1}}\right)^{-\left(\Delta_{2}+\Delta_{3}-\Delta_{1}\right) / 2} \Gamma\left(\frac{\Delta_{2}+\Delta_{3}-\Delta_{1}}{2}+1\right) \lambda_{I_{1} I_{2} I_{3}}^{L_{1} L_{2} L_{3}}
\end{gathered}
$$

with

$$
\lambda_{I_{1} I_{2} I_{3}}^{L_{1} L_{2} L_{3}}={ }_{(1)}\left\langle I_{1},\left.L_{1}\right|_{(2)}\left\langle I_{2},\left.L_{2}\right|_{(3)}\left\langle I_{3}, L_{3}\left|\frac{\sqrt{J_{1} J_{2} J_{3}}}{N} R\left(h_{s}^{(2)}+h_{s}^{(3)}-h_{s}^{(1)}\right)\right| \tilde{V}_{0}\right\rangle,\right.\right.
$$

and the 3 -point interaction term of the action is

$$
S_{3}=\frac{1}{2} \int d \tau_{(1)}\left\langle\overline { \psi } | _ { ( 2 ) } \left\langle\left.\psi\right|_{(3)}\left\langle\psi\left|\frac{\sqrt{J_{1} J_{2} J_{3}}}{N}\left(h_{s}^{(2)}+h_{s}^{(3)}-h_{s}^{(1)}\right)\right| V_{0}\right\rangle+h . c . .\right.\right.
$$

This is the main prediction of the present paper. By construction, this gives the same CFT coefficients for the purely supergravity modes. But for higher stringy modes, the correction factor would play an important role for impurity non-preserving processes. The appearance of the factor $f$ with non-trivial $\alpha^{\prime}$ dependence is not surprising if we

recall that the origin of the factor $\left(\frac{J_{2} J_{3}}{J_{1}}\right)^{-\left(\Delta_{2}+\Delta_{3}-\Delta_{1}\right) / 2}$ is the relation between the length $\delta$ and the cutoff with respect to the $\tau$ integration, as exhibited in the integral (2.35). Our prediction indicates that the nonlocality of the vertex is essentially represented by a rescaling $\delta \rightarrow \delta \sqrt{f}$. Finally, we also mention that this interaction vertex is not $\mathrm{SO}(8)$ symmetric, contrary to a possibility suggested in [4]. The origin of this phenomenon is the asymmetric roles played by scalar and vector excitations from the viewpoint of boundary theory, as we have analyzed in the previous subsection.

\section{Holographic string field theory}

In our first work [4 on the PP-wave holography, we have emphasized that string field theory describing the plane-wave background cannot be constructed uniquely by the requirement of supersymmetry alone. Our claim was that there should exist a unique 'holographic' string field theory which realizes our holographic relation. We now have a more concrete constraint for the holographic string field that it should have the 3-point vertex (3.85) when restricted to the bosonic supergravity sector. 
There are basically two different proposals for 3-point vertex, conforming to the requirement of supersymmetry algebra $\left\{Q_{a}, Q_{\dot{a}}^{\dagger}\right\}=2 \delta_{a \dot{a}} H$ up to the first order with respect to the string coupling. Let us first briefly review them. The most familiar is the one which was first proposed in [16, 17] as a generalization of the light-cone string field theory in flat spacetime, as constructed by Brink, Green, and Schwarz long time ago. This proposal was corrected and established in subsequent works [18, 19, 20, 21]. We denote this vertex by

$$
\left|H_{3}\right\rangle_{S V}=P_{S V}|E\rangle
$$

where $|E\rangle$ is the overlap vertex including both bosonic and fermionic oscillators. The explicit form of the prefactor $P_{S V}$ is given in the Appendix $\mathrm{B}$, where all of the other necessary definitions and formulas of string field theory are summarized, in order to make the present paper reasonably self-contained. It has been shown that this vertex has a nontrivial relation with the matrix of operator mixing which appears in the perturbative computation of anomalous dimensions on the gauge-theory side. We will come back to this point later.

Another version of the 3-point vertex, which was proposed [26] later in connection with the duality relation of our type, takes the following form

$$
\left|H_{3}\right\rangle_{D}=\left(H_{2}^{(2)}+H_{2}^{(3)}-H_{2}^{(1)}\right)|E\rangle
$$

where

$$
H_{2}^{(r)}=\frac{1}{\left|\alpha_{(r)}\right|} \sum_{n=-\infty}^{\infty} \omega_{n}^{(r)}\left(a_{n}^{(r) \dagger} a_{n}^{(r)}+b_{n}^{(r) \dagger} b_{n}^{(r)}\right) \quad \text { with } \quad \omega_{n}^{(r)}=\sqrt{n^{2}+\left(\mu \alpha_{(r)}\right)^{2}}
$$

are the free Hamiltonian operators. Here the string-length parameters are chosen such that $\alpha_{(1)}+\alpha_{(2)}+\alpha_{(3)}=0$ with $\alpha_{(2)}, \alpha_{(3)}>0$ and $\alpha_{(1)}<0$ to be consistent with our conventions used in the foregoing analyses. Note that this Hamiltonian coincides with the total free Hamiltonian $h_{s v}$, (3.71), of the previous section when it is restricted to supergravity modes with the identification $\mu=1 / R$. The form (4.2) is the simplest realization of the way which was discussed in our first work [4 to obtain susy-compatible interaction vertices. In particular, it satisfies the $\mathrm{SO}(8)$ symmetry suggested there. The authors of [26] has shown that this vertex gives nontrivial matrix elements in the leading order of the $1 / \mu$-expansion for impurity preserving processes, following the duality relation 
of our type

$$
\left(\Delta_{2}+\Delta_{3}-\Delta_{1}\right) C_{123}=\frac{\sqrt{J_{1} J_{2} J_{3}}}{N} \frac{1}{\mu}\left\langle 1,2,3 \mid H_{3}\right\rangle,
$$

when the mixing of gauge-theory operators is ignored. This duality relation itself for impurity-preserving processes was first proposed in [29] from a viewpoint which is entirely different from ours, and was actually abandoned later since it turned out that the relation was not appropriate for the original purpose after correcting a sign error in some earlier versions of works on PP-wave string field theory. This relation is in fact obtained from our holographic relation in the limit of large $\mu$ for arbitrary 3-point processes when the number of impurities is preserved. We stress that in the light of our works, the more general relation summarized in (3.82) (3.84) has a firm physical foundation from the holographic principle. We should also recall that we are using the Euclidean picture of the tunneling and hence this form of the interaction proportional to energy difference must not be thrown away by a possible nonlinear unitary transformation: The 'energies' are not conserved for the Euclidean S-matrix. For a detailed discussion of our S-matrix picture, we invite the reader to [4].

These two candidates for the 3-point vertex conform to different duality relations connecting bulk and boundary. The first type $\left|H_{3}\right\rangle_{S V}$ does not fit to our holographic relation. The second type $\left|H_{3}\right\rangle_{D}$ does not either, though motivated by (4.4), since for all of our arguments from section 2 and 3, the gauge-theory operators have to be definite conformal operators of which 2-point functions are diagonalized and 3-point correlators take the standard conformal form (3.8). However, it is important here to recall that the requirement of supersymmetry algebra to the first order in the string coupling puts only a linear constraint. Hence, any linear combination of two possible vertices with some global coefficients depending only on string length parameters and the curvature parameter $\sqrt{\alpha^{\prime}} \mu \sim \sqrt{\alpha^{\prime}} / R$ can be an allowed candidate for the 3-point vertex.

We can now study whether it is possible to obtain our effective action (3.85) for supergravity modes from these vertices. Let us first examine how the above two vertices behave when they are restricted to purely supergravity modes. For purely bosonic external states, they take the following forms

$$
\left|H_{3}\right\rangle_{S V} \Rightarrow \frac{1}{2}\left[\sum_{i=5}^{8}\left(\left(X_{\mathrm{I}}^{i}\right)^{2}-\left(X_{\mathrm{II}}^{i}\right)^{2}\right)-\sum_{\mu=1}^{4}\left(\left(X_{\mathrm{I}}^{\mu}\right)^{2}-\left(X_{\mathrm{II}}^{\mu}\right)^{2}\right]\right)\left|E_{a}\right\rangle
$$




$$
\begin{aligned}
& =\sum_{r=1}^{3} \sum_{i=5}^{8}\left(\sum_{m=0}^{\infty} \frac{\omega_{m}^{(r) i}}{\alpha_{(r)}} a_{m}^{(r) i \dagger} a_{m}^{(r) i}-\sum_{m=1}^{\infty} \frac{\omega_{m}^{(r) i}}{\alpha_{(r)}} a_{-m}^{(r) i \dagger} a_{-m}^{(r) i}\right)\left|E_{a}\right\rangle \\
& -\sum_{r=1}^{3} \sum_{i=1}^{4}\left(\sum_{m=0}^{\infty} \frac{\omega_{m}^{(r)}}{\alpha_{(r)}} a_{m}^{(r) i \dagger} a_{m}^{(r) i}-\sum_{m=1}^{\infty} \frac{\omega_{m}^{(r) i}}{\alpha_{(r)}} a_{-m}^{(r) i \dagger} a_{-m}^{(r) i}\right)\left|E_{a}\right\rangle, \\
\left|H_{3}\right\rangle_{D} & \Rightarrow \frac{1}{2}\left[\sum_{i=5}^{8}\left(\left(X_{\mathrm{I}}^{i}\right)^{2}+\left(X_{\mathrm{II}}^{i}\right)^{2}\right)+\sum_{\mu=1}^{4}\left(\left(X_{\mathrm{I}}^{\mu}\right)^{2}+\left(X_{\mathrm{II}}^{\mu}\right)^{2}\right)\right]\left|E_{a}\right\rangle \\
& =\sum_{r=1}^{3} \sum_{i=1}^{8}\left(\sum_{m=0}^{\infty} \frac{\omega_{m}^{(r)}}{\alpha_{(r)}} a_{m}^{(r) i \dagger} a_{m}^{(r) i}+\sum_{m=1}^{\infty} \frac{\omega_{m}^{(r)}}{\alpha_{(r)}} a_{-m}^{(r) i \dagger} a_{-m}^{(r) i}\right)\left|E_{a}\right\rangle .
\end{aligned}
$$

The state $\left|E_{a}\right\rangle$ is the bosonic part of the overlap state. The expression $X_{\mathrm{I}}$ and $X_{\mathrm{II}}$, defined in the Appendix B, contain the oscillators of only cos and sin modes, respectively, which are represented by the creation-and-annihilation operator of positive $\left(a_{n}^{(r)}\right)$ and negative $\left(a_{-n}^{(r)}\right)$ indices, respectively. As briefly discussed there, the factorization formula for the Neumann matrices [23, 18, allows us to derive the above simple expressions in terms of world-sheet energies $\omega_{m}^{r}$.

By restricting to the zero modes, we see that neither of them separately reduces to the effective action of the previous section. However, it is now evident that if we combine them with equal weights we can get the desired form

$$
\begin{array}{r}
\left|H_{3}\right\rangle_{h} \equiv \frac{1}{2}\left(\left|H_{3}\right\rangle_{S V}+\left|H_{3}\right\rangle_{D}\right) \Rightarrow\left(\sum_{i=5}^{8}\left(X_{\mathrm{I}}^{i}\right)^{2}+\sum_{i=1}^{4}\left(X_{\mathrm{II}}^{i}\right)^{2}\right)\left|E_{a}\right\rangle \\
=\sum_{r=1}^{3}\left(\sum_{i=5}^{8} \sum_{m=0}^{\infty} \frac{\omega_{m}^{(r)}}{\alpha_{(r)}} a_{m}^{(r) i \dagger} a_{m}^{(r) i}+\sum_{i=1}^{4} \sum_{m=1}^{\infty} \frac{\omega_{m}^{(r)}}{\alpha_{(r)}} a_{-m}^{(r) i \dagger} a_{-m}^{(r) i}\right)\left|E_{a}\right\rangle
\end{array}
$$

which in the zero-mode sector reduces to the form $\left(h_{s}^{(2)}+h_{s}^{(3)}-h_{s}^{(1)}\right)\left|V_{0}\right\rangle$, involving only the scalar oscillators in the prefactor, as derived in the previous section. Apart from the overall normalization fixed by comparing with (3.85), this combination is unique. Therefore, we have arrived at the uniquely possible string field 3-point interaction term which is consistent with the conclusion that we have reached by foregoing discussions.

We note that when restricted to the scalar modes the form (4.7) coincides with the one suggested previously in [36] as the possible prefactor which is compatible with the holographic relation (4.4). Namely, they have shown that this gives the correct CFT coefficients after taking into account the operator mixing for scalar operators with two impurities. The result (4.7) shows that the scalar prefactor indeed consists only of the 
cos modes as they have proposed, while actually the vector prefactor must consist only of the sin modes, in order to be consistent with supersymmetry.

Thus the conclusion of this section is that the holographic string field theory is given in the following form up to the first order in the string coupling

$$
\begin{gathered}
S_{h}=S_{h, 2}+S_{h, 3}, \\
S_{h, 2}=\int d \tau\left[\frac{1}{2}\left(\left\langle\bar{\psi}\left|\partial_{\tau}\right| \psi\right\rangle-\frac{1}{2}\left(\partial_{\tau}\langle\bar{\psi}|\right)|\psi\rangle+\left\langle\bar{\psi}\left|H_{2}\right| \psi\right\rangle\right],\right. \\
S_{h, 3}=\frac{1}{2 N} \int d \tau_{(1)}\left\langle\overline { \psi } | _ { ( 2 ) } \left\langle\left.\psi\right|_{(3)}\left\langle\psi\left|\sqrt{J_{1} J_{2} J_{3}}\right| H_{3}\right\rangle_{h}+\right.\right.\text { h.c. }
\end{gathered}
$$

The integral over $J_{i}$ under the condition $J_{1}=J_{2}+J_{3}$ is implicit as before.

This action has no $\mathrm{SO}(8)$ symmetry, nor even the $Z_{2}$ symmetry. We also note that the holographic string field theory does not directly reduce to the familiar Green-Schwarz form in the flat limit $R \rightarrow \infty$. This was the case already in the supergravity sector. Since the AdS/CFT correspondence between bulk gravity (closed string theory) and CFT on the boundary is a concept which requires a global consideration of the whole AdS spacetime, the fact that the flat limit is not direct is not surprising.

\section{Relation with other possible duality maps}

In the present section, we discuss the relation of our results to other proposals for duality maps, especially the one advocated in refs. [32]. As we have already mentioned, it has been shown that the 3-point vertex $\left|H_{3}\right\rangle_{S V}$ of the first type has a close connection with the matrix of operator mixing in the perturbative computation of anomalous dimensions. It is indeed reasonable to assume that there exists a quantity in the bulk which plays the role of the operator mixing. The operator mixing is necessarily associated with perturbative renormalization procedure in higher orders with respect both to gauge coupling and to genus expansion in our situation where there is a large degeneracy with respect to conformal dimensions at the lowest order.

The first indication for the necessity of taking into account the operator mixing comes from the behavior of 3-point functions at the first order in $\lambda^{\prime} \equiv \lambda / J^{2}$. Take for instance the simplest case of the 3 -point function of operators with two different impurities $(i \neq j)$,

$$
\left\langle\bar{O}_{i j, n}^{J}\left(x_{1}\right) O_{i j, m}^{y J}\left(x_{2}\right) O_{\text {vac }}^{(1-y) J}\left(x_{3}\right)\right\rangle=
$$




$$
g_{2} C_{n ; m y}\left[1-\lambda^{\prime}\left(a_{n ; m y} \log \left(x_{12} \Lambda\right)^{2}+b_{n ; m y} \log \left(\frac{x_{31} x_{12} \Lambda}{x_{23}}\right)\right)\right]
$$

where

$$
O_{i j, n}^{J}=\frac{1}{\sqrt{J N^{J+2}}} \sum_{l=0}^{J} \mathrm{e}^{\frac{2 \pi i n l}{J}} \operatorname{Tr}\left(\phi_{i} Z^{l} \phi_{j} Z^{J-l}\right)
$$

and $O_{\text {vac }}^{J}$ is just the BMN operator corresponding to the ground state, with

$$
a_{n ; m y}=m^{2} / y^{2}, \quad b_{n ; m y}=n(n-m / y), \quad \text { and } \quad C_{n ; m y}=\sqrt{\frac{1-y}{y J}} \frac{\sin ^{2}(\pi n y)}{\pi^{2}\left(n-\frac{m}{y}\right)^{2}} .
$$

For notational brevity, in the present section, we suppress the classical part (namely, the zero-th order in $\lambda^{\prime}$ ) of spacetime dependence for gauge-theory correlation functions. Note that $a_{n ; m y}$ is noting but the anomalous dimension of $O_{m}^{y J}$ since it comes from Feynman diagrams where the interaction occurs only between $O_{n}^{J}\left(x_{1}\right)$ and $O_{m}^{y J}\left(x_{2}\right)$, while $b_{n ; m y}$ comes from graphs where the interaction occurs among all three operators $O_{n}^{J}\left(x_{1}\right), O_{m}^{y J}\left(x_{2}\right)$ and $O_{\text {vac }}^{(1-y) J}\left(x_{3}\right)$. This form, however, does not take the standard form of a 3-point correlation function of conformal operators. In other words, to this first order in $\lambda^{\prime}$, the BMN operator $O_{i j, n}^{J}$ cannot be regarded as a conformal operator characterized by the standard conformal transformation property.

As is well known now, this difficulty is resolved if we take into account the operator mixing with multi-trace operators. Consider a $2 \times 2$ matrix of two-point functions of operators,

$$
O_{i j, n}^{J}=\frac{1}{\sqrt{J N^{J+2}}} \sum_{l=0}^{J} \mathrm{e}^{\frac{2 \pi i n l}{J}} \operatorname{Tr}\left(\phi_{i} Z^{l} \phi_{j} Z^{J-l}\right) \quad \text { and } \quad T_{i j, n}^{J, y}=: O_{i j, n}^{y \cdot J} O_{\mathrm{vac}}^{(1-y) \cdot J}: .
$$

With respect to the genus-expansion parameter $g_{2} \equiv J^{2} / N$, the diagonal elements begin from the zero-th order, while the off-diagonal elements, being interpreted as a 2-body to 1body (or 1-body to 2-body) process, starts from the first order. Therefore, we can express the general structure of this matrix to the first order both in $\lambda^{\prime}$ and $g_{2}$ as [28, 29, 30, 31.

$$
\left\langle\bar{O}_{A}(0) O_{B}(x)\right\rangle \equiv G_{A B}+\lambda^{\prime} \Gamma_{A B} \log (x \Lambda)^{-2},
$$

with

$$
\begin{aligned}
G_{A B} & =\left(\begin{array}{cc}
\delta_{m n} & 0 \\
0 & \delta_{m n} \delta_{y z}
\end{array}\right)+g_{2}\left(\begin{array}{cc}
0 & C_{n ; m y} \\
C_{n ; m y} & 0
\end{array}\right) \\
\Gamma_{A B} & =\left(\begin{array}{cc}
n^{2} \delta_{n m} & 0 \\
0 & \frac{m^{2}}{y^{2}} \delta_{n m} \delta_{y z}
\end{array}\right)+g_{2}\left(\begin{array}{cc}
0 & \left(a_{n ; m y}+b_{n ; m y}\right) C_{n ; m y} \\
\left(a_{n ; m y}+b_{n ; m y}\right) C_{n ; m y} & 0
\end{array}\right)
\end{aligned}
$$


Note hat the 2-point functions between a single-trace and a double-trace operator are obtained by taking the limit $x_{23} \sim 1 / \Lambda \rightarrow 0$, and also that $1 / \Lambda$ is identified with the short-distance cutoff parameter associated with loops in the sense of Feynman diagrams.

The specific ansatz proposed in 32 in order to relate this mixing matrix to string-field theory vertex $\left|H_{3}\right\rangle_{S V}$ is as follows. We first transform the basis for these operators by $\widetilde{O}_{A}=\widetilde{U}_{A B} O_{B}$ such that

$$
\left\langle\overline{\widetilde{O}}_{A}(0) \widetilde{O}_{B}(x)\right\rangle=\delta_{A B}+\mathcal{O}\left(\lambda^{\prime}\right)
$$

However, this requirement alone does not determine the basis completely. To restrict the basis further the authors demand that the transformation should be symmetric and real. Then, the transformation matrix $\tilde{U}$ can be fixed to the present order $\mathcal{O}\left(g_{2}\right)$ as

$$
\tilde{U}=1-\frac{g_{2}}{2} G^{(1)}
$$

where $G^{(1)}$ is the $\mathcal{O}\left(g_{2}\right)$ part of $G_{A B}$. By this change of the basis, the matrix $\Gamma_{A B}$ is transformed to

$$
\Gamma^{\prime}=\Gamma^{(0)}+g_{2} \Gamma^{(1)}-\frac{g_{2}}{2}\left\{\Gamma^{(0)}, G^{(1)}\right\}
$$

to the present order of approximation. The observation of [32] is that the off-diagonal part of this matrix coincides with the matrix elements of the interaction vertex of the first type, namely, $\left|H_{3}\right\rangle_{S V}$. This has been confirmed in several cases, for instance, for scalar impurities in [32, 33, 14, and for other cases including vectors and fermions in [14, 39].

For the supergravity chiral operators, the mixing matrix $\Gamma_{A B}$ vanishes. In connection with this, we note that in this case the matrix $G_{A B}$ should not be interpreted as representing operator mixing, as we will touch briefly below. This is as it should be, since the 3-point functions of the chiral supergravity operators are not renormalized and hence take the standard conformally-invariant form even after including the higher-order effects.

We now show that this ansatz can be understood as a consequence of our holographic string field theory, given the observation of [26]. In other words, all existing duality relations are actually compatible. Since our results are based on a clear spacetime picture which is lacking unfortunately in other approaches, checking the consistency with more formal correspondences seems to provide a useful guide for obtaining a unified viewpoint on the holography in the plane-wave limit. 
Let us consider a general class of three general operators $O_{1}\left(x_{1}\right), O_{2}\left(x_{2}\right)$ and $O_{3}\left(x_{3}\right)$, which have the classical conformal dimensions $\Delta_{r}^{\langle 0\rangle}$ satisfying the condition of degeneracy $\Delta_{1}^{\langle 0\rangle}=\Delta_{2}^{\langle 0\rangle}+\Delta_{3}^{\langle 0\rangle}$. The number of impurities contained in $O_{1}$ is thus equal to the sum of those in $\mathrm{O}_{2}$ and $\mathrm{O}_{3}$. Here and in what follows, we use the subscript $\langle 0\rangle,\langle 1\rangle$, etc to denote the order with respect to $\lambda^{\prime}$. The coupling constant $g_{2}$ with respect to which the order is denoted by the usual subscript $(0),(1)$, etc is factored out in order to make clear the order of the quantities we are dealing with. The 3-point function takes the following general form

$$
\begin{aligned}
& \left\langle\bar{O}_{1}\left(x_{1}\right) O_{2}\left(x_{2}\right) O_{3}\left(x_{3}\right)\right\rangle \\
& \quad=g_{2} C_{123}^{\langle 0\rangle}\left[1-\lambda^{\prime}\left\{\Delta_{2}^{\langle 1\rangle} \log \left(x_{12} \Lambda\right)^{2}+\Delta_{3}^{\langle 1\rangle} \log \left(x_{13} \Lambda\right)^{2}+b_{123} \log \left(\frac{x_{31} x_{12} \Lambda}{x_{23}}\right)\right\}\right]
\end{aligned}
$$

where $C_{123}^{\langle 0\rangle}$ denotes the 3-point coefficient of the free gauge theory and $\Delta_{r}^{\langle 1\rangle}$ is the anomalous dimension of the operator $O_{r}\left(x_{r}\right)$ to the first order in $\lambda^{\prime}$. As above, this expression in general does not conform to the standard form of the 3-point correlation functions of conformal operators. However, we can easily check that this is the most general form which would lead to the standard form ([5.16) below) after taking into account the operator mixing. In the $\mathcal{O}\left(\lambda^{\prime}\right)$ part on the right hand side of (5.10) , the first term $\Delta_{2}^{\langle 1\rangle} \log \left(x_{12} \Lambda\right)^{2}$ comes from a class of Feynman diagrams in which the interaction occurs only between $\mathrm{O}_{2}\left(x_{2}\right)$ and $O_{1}\left(x_{1}\right)$. Similarly, the second term $\Delta_{3}^{\langle 0\rangle} \log \left(x_{13} \Lambda\right)^{2}$ comes from the ones where the interaction occurs only between $O_{3}\left(x_{3}\right)$ and $O_{1}\left(x_{1}\right)$, and the third term $b_{123} \log \left(\left(x_{31} x_{12} \Lambda\right) / x_{23}\right)$ from the remaining ones, where the interaction occurs among all three operators. Supposing that the double-trace operator in consideration is obtained from the product of $\mathrm{O}_{2}\left(x_{2}\right)$ and $O_{3}\left(x_{3}\right)$ by taking the limit $x_{23} \sim 1 / \Lambda$, the matrices $G_{A B}$ and $\Gamma_{A B}$ in the subspace of operators $O_{1}(0)$ and : $O_{2}(x) O_{3}(x)$ : takes the form

$$
\begin{aligned}
G_{A B}= & \left(\begin{array}{ll}
1 & 0 \\
0 & 1
\end{array}\right)+g_{2}\left(\begin{array}{cc}
0 & C_{123}^{\langle 0\rangle} \\
C_{123}^{\langle 0\rangle} & 0
\end{array}\right), \\
\Gamma_{A B}= & \left(\begin{array}{cc}
\Delta_{1}^{\langle 1\rangle} & 0 \\
0 & \Delta_{2}^{\langle 1\rangle}+\Delta_{3}^{\langle 1\rangle}
\end{array}\right) \\
& \quad+g_{2}\left(\begin{array}{cc}
\left(\Delta_{2}^{\langle 1\rangle}+\Delta_{3}^{\langle 1\rangle}+b_{123}\right) C_{123}^{\langle 0\rangle} & \left(\Delta_{2}^{\langle 1\rangle}+\Delta_{3}^{\langle 1\rangle}+b_{123}\right) C_{123}^{\langle 0\rangle}
\end{array}\right),
\end{aligned}
$$


where we have taken into account the fact that the $\mathcal{O}\left(g_{2}^{0}\right)$ part of $\Gamma_{A B}$ gives the anomalous dimensions of $\mathrm{O}_{1}$ and $: \mathrm{O}_{2} \mathrm{O}_{3}$ : In the case of two different scalar impurities discussed above, $\Delta_{2}^{\langle 1\rangle}=m^{2} / y^{2}, \Delta_{3}^{\langle 1\rangle}=0, \Delta_{1}^{\langle 1\rangle}=n^{2}, b_{123}=b_{n ; m y}=n(n-m / y)$, and $C_{123}^{\langle 0\rangle}=C_{n ; m y}$.

Now, in order to extract the correct CFT coefficient with the operator mixing being taken into account, we introduce the transformation matrix $U_{A B}$ which diagonalizes the matrix $\left\langle\bar{O}_{A}(0) O_{B}(x)\right\rangle$ as a whole. Namely, contrary to the previous $\tilde{U}$, both of the matrices $G_{A B}$ and $\Gamma_{A B}$ are simultaneously diagonalized. It takes the form

$$
U=\left(\begin{array}{ll}
1 & 0 \\
0 & 1
\end{array}\right)+g_{2}\left(\begin{array}{cc}
0 & D_{123} \\
E_{123} & 0
\end{array}\right)
$$

where

$$
D_{123}=\frac{\Delta_{2}^{\langle 1\rangle}+\Delta_{3}^{\langle 1\rangle}-\Delta_{1}^{\langle 1\rangle}+b_{123}}{\Delta_{1}^{\langle 1\rangle}-\Delta_{2}^{\langle 1\rangle}-\Delta_{3}^{\langle 1\rangle}} C_{123}^{\langle 0\rangle}, \quad E_{123}=-\frac{b_{123}}{\Delta_{1}^{\langle 1\rangle}-\Delta_{2}^{\langle 1\rangle}-\Delta_{3}^{\langle 1\rangle}} C_{123}^{\langle 0\rangle} .
$$

Then the 3-point function of the operators $O_{A}^{\prime}=U_{A B} O_{B}$ in the new basis is given by

$$
\begin{aligned}
\langle & \left.\bar{O}_{1}^{\prime}\left(x_{1}\right) O_{2}^{\prime}\left(x_{2}\right) O_{3}^{\prime}\left(x_{3}\right)\right\rangle \\
= & \left\langle\bar{O}_{1}\left(x_{1}\right) O_{2}\left(x_{2}\right) O_{3}\left(x_{3}\right)\right\rangle+g_{2} D_{12^{\prime} 3^{\prime}}\left\langle\overline{: O_{2^{\prime}} O_{3^{\prime}}:}\left(x_{1}\right) O_{2}\left(x_{2}\right) O_{3}\left(x_{3}\right)\right\rangle+\mathcal{O}\left(g_{2}^{2}\right) \\
= & g_{2}\left(C_{123}^{\langle 0\rangle}+D_{123}\right) \\
& -\lambda^{\prime} g_{2}\left[\left\{2 \Delta_{2}^{\langle 1\rangle}\left(C_{123}^{\langle 0\rangle}+D_{123}\right)+b_{123} C_{123}^{\langle 0\rangle}\right\} \log \left(x_{12} \Lambda\right)\right. \\
& \left.\quad+\left\{2 \Delta_{3}^{\langle 1\rangle}\left(C_{123}^{\langle 0\rangle}+D_{123}\right)+b_{123} C_{123}^{\langle 0\rangle}\right\} \log \left(x_{31} \Lambda\right)-b_{123} C_{123}^{\langle 0\rangle} \log \left(x_{23} \Lambda\right)\right] .
\end{aligned}
$$

Here, the mixing effect does not affect the third term $\log \left(x_{23} \Lambda\right)$, since a correlation function which contains a double trace operator at $x_{2}$ or $x_{3}$ gives a $\mathcal{O}\left(g_{2}\right)$ contribution by itself. Taking into account the definition of $D_{123}$, we then obtain the following result regardless of the expression of $b_{123}$, which is consistent with the canonical form of the 3-point function for operators with (anomalous) conformal dimensions $\Delta_{1}^{\langle 1\rangle}, \Delta_{2}^{\langle 1\rangle}$, and $\Delta_{3}^{\langle 1\rangle}$, respectively:

$$
\begin{aligned}
& \left\langle\bar{O}_{1}^{\prime}\left(x_{1}\right) O_{2}^{\prime}\left(x_{2}\right) O_{3}^{\prime}\left(x_{3}\right)\right\rangle \\
& =g_{2} C_{123}\left[1-\lambda^{\prime}\left\{\left(\Delta_{1}^{\langle 1\rangle}+\Delta_{2}^{\langle 1\rangle}-\Delta_{3}^{\langle 1\rangle}\right) \log \left(x_{12} \Lambda\right)\right.\right. \\
& \left.\left.+\left(\Delta_{3}^{\langle 1\rangle}+\Delta_{1}^{\langle 1\rangle}-\Delta_{2}^{\langle 1\rangle}\right) \log \left(x_{31} \Lambda\right)+\left(\Delta_{2}^{\langle 1\rangle}+\Delta_{3}^{\langle 1\rangle}-\Delta_{1}^{\langle 1\rangle}\right) \log \left(x_{23} \Lambda\right)\right\}\right]
\end{aligned}
$$

where the true CFT coefficient $C_{123}=C_{123}^{\langle 0\rangle}+D_{123}$ is expressed in terms of $C_{123}^{\langle 0\rangle}$ and $b_{123}$ as

$$
C_{123}=\frac{\lambda^{\prime} b_{123} C_{123}^{\langle 0\rangle}}{\Delta_{1}-\Delta_{2}-\Delta_{3}}
$$


Here, we have used the relation $\Delta_{1}-\Delta_{2}-\Delta_{3}=\lambda^{\prime}\left(\Delta_{1}^{\langle 1\rangle}-\Delta_{2}^{\langle 1\rangle}-\Delta_{3}^{\langle 1\rangle}\right)$, which is valid for impurity-preserving processes. Though we have taken into account the operator mixing to higher order with respect to both $g_{2}$ and $\lambda^{\prime}$, the correction to the CFT coefficients thus starts from the lowest order, namely the same order as $C_{123}^{\langle 0\rangle}$.

Note that this argument can be applied for any kind of impurities, except for the case of pure chiral operators where $a_{123}, b_{123}$ and $\Delta_{i}^{\langle 0\rangle}$ all vanish: The relation (15.17) holds with different $b_{n ; m y}$ 's depending on impurities [14, 39]. Also, for the chiral operators of supergravity, this procedure would lead to a nonsensical result $C_{123}=0$, indicating that for the supergravity BMN operators the matrix $G_{A B}$ cannot be regarded as the mixing matrix.

By identifying the true CFT coefficient with the 3-point vertex $\left|H_{3}\right\rangle_{h}$ in accordance with our result for the holographic string field theory, we must have

$$
\frac{\sqrt{J_{1} J_{2} J_{3}}}{N}\left\langle 1,2,3\left|\left(\frac{1}{2 \mu}\left|H_{3}\right\rangle_{D}+\frac{1}{2 \mu}\left|H_{3}\right\rangle_{S V}\right)=-g_{2} \lambda^{\prime} b_{123} C_{123}^{\langle 0\rangle} .\right.\right.
$$

On the other hand, the result of [26] indicates that

$$
\frac{\sqrt{J_{1} J_{2} J_{3}}}{N} \frac{1}{\mu}\left\langle 1,2,3 \mid H_{3}\right\rangle_{D}=g_{2}\left(\Delta_{2}+\Delta_{3}-\Delta_{1}\right) C_{123}^{\langle 0\rangle} .
$$

The off-diagonal matrix elements of the $\Gamma$-matrix in the particular basis which makes the partial diagonalization are given in the form

$$
\lambda^{\prime} \Gamma_{\text {off }}^{\prime}=\frac{1}{2}\left(\Delta_{2}+\Delta_{3}-\Delta_{1}\right) C_{123}^{\langle 0\rangle}+\lambda^{\prime} b_{123} C_{123}^{\langle 0\rangle} .
$$

Using the equations (5.17), (5.18) and (5.19), we finally find

$$
g_{2} \lambda^{\prime} \Gamma_{\text {off }}^{\prime}=-\frac{\sqrt{J_{1} J_{2} J_{3}}}{N} \frac{1}{2 \mu}\left\langle 1,2,3 \mid H_{3}\right\rangle_{S V} .
$$

This is nothing but the claim made in [32, 33], except for the overall factor $-1 / 2$. This factor can be understood from the difference of normalization. Our convention differs just by this factor from the one adopted in the literature discussing this subject; see, for example, (B.14) and (B.9) in 39.

It should be remarked that the above relation of the 3-point vertex $\left|H_{3}\right\rangle_{S V}$ with the operator mixing in perturbation theory at the boundary is intrinsically restricted to the processes where the numbers of impurities are conserved. For this particular class of 
processes, our argument clarified why the correct interaction vertex of the holographic string field theory must be the particular combination of the two types of string interaction vertices: Roughly speaking, the part $\left|H_{3}\right\rangle_{D}$ describes the 'bare' part of the interaction of BMN operators, while the part $\left|H_{3}\right\rangle_{S V}$ describes the mixing among them. Both are necessary for describing the processes in the bulk, corresponding to a propagation of them from boundary to boundary along the tunneling null geodesic. Note that the observation of [26] that the string overlap vertex, the bare part of the interaction, in the large $\mu$ limit precisely corresponds to the free-field contraction is also quite natural for impurity preserving processes. Through the holographic string field theory, this natural property is related to the specific ansatz relating $\left|H_{3}\right\rangle_{S V}$ to the matrix of operator mixing, which singles out the particular basis of the gauge-theory operators.

We warn the reader, however, that the above intuitive interpretation on the different roles of $\left|H_{3}\right\rangle_{S V}$ and $\left|H_{3}\right\rangle_{D}$ does not apply to more general processes in which the numbers of impurities are not conserved. It is difficult to extend the above argument directly to such cases.

\section{Explicit examples}

The purpose of this section is to present some concrete computations in order to confirm our general discussions. In the present work, for simplicity we restrict ourselves to the cases of two (conserved) impurities, for which we can utilize many results by other authors on the gauge theory side. It is sufficient to focus on the cases of vector, mixed scalarvector, and fermionic impurities, since as we have mentioned before the case of pure scalar impurities has been practically treated in [36] and was shown to be consistent with our holographic relation. We are planning to study more general cases, especially the cases where the numbers of impurities are not conserved, in a forthcoming work. In the present section, we denote the first 4 -vector indices $(i=1, \ldots, 4)$ by Greek letters $(\mu, \nu)$ for a clear discrimination between the vector and scalar directions.

\subsection{Vector impurities}

Let us begin from the BMN operators with two vector impurities. The CFT coefficients on the gauge theory side have been already computed in ref.[14. Non-trivial processes 
are listed bellow for $\left(\Delta_{2}+\Delta_{3}-\Delta_{1}\right) C_{123}$ :

$$
\begin{array}{ll}
\mu_{m} \nu_{-m}+\operatorname{vac} \rightarrow \mu_{n} \nu_{-n}: & -\lambda^{\prime} C_{\mathrm{vac}} \frac{\sin ^{2}(\pi n y)}{y \pi^{2}} \frac{m / y}{n-m / y} \\
\nu_{m} \mu_{-m}+\operatorname{vac} \rightarrow \mu_{n} \nu_{-n}: & \lambda^{\prime} C_{\mathrm{vac}} \frac{\sin ^{2}(\pi n y)}{y \pi^{2}} \frac{m / y}{n+m / y} \\
\mu_{m} \mu_{-m}+\operatorname{vac} \rightarrow \nu_{n} \nu_{-n}: & -\frac{\lambda^{\prime}}{2} C_{\mathrm{vac}} \frac{\sin ^{2}(\pi n y)}{y \pi^{2}} \\
\mu_{m} \mu_{-m}+\operatorname{vac} \rightarrow \mu_{n} \mu_{-n}: & -\frac{\lambda^{\prime}}{2} C_{\mathrm{vac}} \frac{\sin (\pi n y)}{y \pi^{2}} \frac{n^{2}+3 m^{2} / y^{2}}{n^{2}-m^{2} / y^{2}},
\end{array}
$$

where the left hand side represents symbolically the processes with two vector impurities with $\mu$ and $\nu$ supposed to denote different vector indices. We have already used the fact that the difference of the conformal dimensions, $\Delta_{2}+\Delta_{3}-\Delta_{1}$, is given by $\lambda^{\prime}\left(m^{2} / y^{2}-n^{2}\right)$ with $y \equiv J_{2} / J_{1}$ at the leading order of small $\lambda^{\prime}=1 /\left(\mu \alpha_{(1)}\right)^{2}$. The mode numbers $n$ and $m$ are supposed to satisfy $n>0$ and $m \geq 0$, respectively. The overall numerical constant $C_{\text {vac }}$ is the 3 -point function for vacuum BMN operators, $O_{\text {vac }}^{J_{i}} \equiv\left(J_{i} N^{J_{i}}\right)^{-1 / 2} \operatorname{Tr}\left(Z^{J_{i}}\right)$ with $J_{2}+J_{3}=J_{1}$ :

$$
C_{\mathrm{vac}}=\frac{\sqrt{J_{1} J_{2} J_{3}}}{N}=g_{2} \frac{\sqrt{y(1-y)}}{\sqrt{J_{1}}} .
$$

Note that the normalization constant of the BMN operator has always been chosen such that two-point functions take the form $\left\langle\bar{O}^{J}(x) O^{J}(0)\right\rangle=1 /|x|^{2 J}$. This overall factor coincides with the corresponding factor for the 3-point vertex of the holographic string field theory, as determined in the previous section on the basis of the comparison with the supergravity analysis.

The 3-point vertex on the string-theory side which should be compared with is the one with the prefactor with only sin modes, since the scalar part $X_{\mathrm{I}}^{2}$ vanishes for these cases,

$$
\begin{aligned}
X_{\mathrm{II}}^{2}|E\rangle= & -\sum_{r, s=1}^{3} \sum_{m, n=1}^{\infty} \frac{\omega_{m}^{(r)}}{\alpha_{(r)}}\left(\widetilde{N}_{m n}^{r s}-\widetilde{N}_{m-n}^{r s}\right)\left(\alpha_{m}^{(r) \dagger} \alpha_{n}^{(s) \dagger}+\alpha_{-m}^{(r) \dagger} \alpha_{-n}^{(s) \dagger}\right)|E\rangle \\
& +\sum_{r, s=1}^{3} \sum_{m, n=1}^{\infty}\left(\frac{\omega_{m}^{(r)}}{\alpha_{(r)}}+\frac{\omega_{n}^{(s)}}{\alpha_{(s)}}\right)\left(\widetilde{N}_{m n}^{r s}-\widetilde{N}_{m-n}^{r s}\right) \alpha_{m}^{(r) \dagger} \alpha_{-n}^{(s) \dagger}|E\rangle,
\end{aligned}
$$

where we have expressed the formula in terms of the exponential basis defined as

$$
\alpha_{0}=a_{0}, \quad \alpha_{n}=\frac{1}{\sqrt{2}}\left(a_{n}-i a_{-n}\right), \quad \alpha_{-n}=\frac{1}{\sqrt{2}}\left(a_{n}+i a_{-n}\right),
$$


which directly corresponds to the momentum basis of the BMN operators on the boundary.

For the first process (6.22), assuming $m$ and $n$ are non-zero, we obtain the following matrix element

$$
\begin{aligned}
& { }_{123}\left\langle v\left|\alpha_{n}^{(1) \mu} \alpha_{-n}^{(1) \nu} \alpha_{m}^{(2) \mu} \alpha_{-m}^{(2) \nu} \frac{1}{2 \mu}\left(X_{\mathrm{II}}^{2}\right)\right| E\right\rangle \\
& =\frac{1}{2 \mu}\left(\frac{\omega_{n}^{(1)}}{\alpha_{(1)}}\left(\widetilde{N}_{n m}^{12}-\widetilde{N}_{n-m}^{12}\right)+\frac{\omega_{m}^{(2)}}{\alpha_{(2)}}\left(\widetilde{N}_{m n}^{21}-\widetilde{N}_{m-n}^{21}\right)\right) \widetilde{N}_{-n-m}^{12} \\
& \quad+\frac{1}{2 \mu}\left(\frac{\omega_{n}^{(1)}}{\alpha_{(1)}}\left(\widetilde{N}_{n m}^{12}-\widetilde{N}_{n-m}^{12}\right)+\frac{\omega_{m}^{(2)}}{\alpha_{(2)}}\left(\widetilde{N}_{m n}^{21}-\widetilde{N}_{m-n}^{21}\right)\right) \widetilde{N}_{n m}^{12}
\end{aligned}
$$

where the second line comes form the case where the oscillators $\alpha_{n}^{(1) \mu}$ and $\alpha_{m}^{(2) \mu}$ are contracted through the prefactor $X_{\mathrm{II}}^{2}$ and the operators $\alpha_{-n}^{(1) \nu}$ and $\alpha_{-m}^{(2) \nu}$ through the overlap $|E\rangle$, while the third line comes form the opposite case. The net results is

$$
\frac{1}{\mu}\left(\frac{\omega_{n}^{(1)}}{\alpha_{(1)}}+\frac{\omega_{m}^{(2)}}{\alpha_{(2)}}\right)\left(\widetilde{N}_{n-m}^{12}-\widetilde{N}_{n m}^{12}\right) \widetilde{N}_{n m}^{12}
$$

Using the explicit form of the Neumann coefficients in the large $\mu$ limit presented in (B.60), we can confirm that this reduces in this limit to the gauge theory result (6.22), after including the above overall factor. As for the special case $m=0$, the absence of zero-mode oscillators in the prefactor $X_{\mathrm{II}}$ leads to the vanishing result, which matches the gauge theory.

The second case (6.23) is related by a change $m \rightarrow-m$ to the first one. On the string theory side, the large $\mu$ behavior of Neumann functions $\widetilde{N}_{m n}^{r s}$ satisfies the same relation with respect to this sign change, as exhibited in (B.60). Thus the first case ensures the correct matching in the second case.

For the third case (6.24), we obtain for $n \neq 0$ and $m \neq 0$,

$$
\begin{aligned}
& { }_{123}\left\langle v\left|\alpha_{n}^{(1) \mu} \alpha_{-n}^{(1) \mu} \alpha_{m}^{(2) \nu} \alpha_{-m}^{(2) \nu} \frac{1}{2 \mu}\left(X_{\mathrm{II}}^{2}\right)\right| E\right\rangle \\
& =-\frac{\omega_{n}^{(1)}}{\mu \alpha_{(1)}}\left(\widetilde{N}_{n n}^{11}-\widetilde{N}_{n-n}^{11}\right) \widetilde{N}_{m-m}^{22}-\frac{\omega_{m}^{(2)}}{\mu \alpha_{(1)}}\left(\widetilde{N}_{m m}^{22}-\widetilde{N}_{m-m}^{22}\right) \widetilde{N}_{n-n}^{11},
\end{aligned}
$$

where the first term in the second line comes from the contraction of $\alpha_{n}^{(1) \mu}$ and $\alpha_{-n}^{(1) \mu}$ through the prefactor $X_{\mathrm{II}}^{2}$ and of $\alpha_{m}^{(2) \nu}$ and $\alpha_{-m}^{(2) \nu}$ through the overlap $|E\rangle$, while the second term comes form the opposite case. Due to the property of the Neumann coefficients, the second term vanishes, and the first term reduces to the field theory result. It is useful to 
notice here that the gauge theory result for vector impurities in the case (6.24) is equal to the scalar correspondent $(\mu, \nu \rightarrow i, j)$ except for the overall sign, and to recall that the scalar case matches the duality relation (4.4) by using the vertex (1/2) $X_{\mathrm{I}}^{2}|E\rangle$. In addition to this, we can easily check that the relation

$$
{ }_{123}\left\langle v\left|\alpha_{n}^{(1) \mu} \alpha_{-n}^{(1) \mu} \alpha_{m}^{(2) \nu} \alpha_{-m}^{(2) \nu}\left(X_{\mathrm{I}}^{2}+X_{\mathrm{II}}^{2}\right)\right| E\right\rangle=\mathcal{O}\left(\frac{1}{\mu^{2}}\right)
$$

is satisfied in the present 'flavor-changing' process. Thus, $X_{\mathrm{II}}^{2}|E\rangle$ can be replaced with $-X_{\mathrm{I}}^{2}|E\rangle$ at the leading order in large $\mu$ limit, confirming the validity of the duality relation. The same is true for the case of $m=0$.

The last case (6.25) is the sum of the above three cases (6.22), (6.23) and (6.24) on the gauge theory side. We can check that the same is true for the expressions on the string side: Indeed,

$$
{ }_{123}\left\langle v\left|\alpha_{n}^{(1) \mu} \alpha_{-n}^{(1) \mu} \alpha_{m}^{(2) \mu} \alpha_{-m}^{(2) \mu} \frac{1}{2 \mu}\left(X_{\mathrm{II}}^{2}\right)\right| E\right\rangle,
$$

is given by summing all possible contractions of $\alpha_{n}^{(1) \mu}, \alpha_{-n}^{(1) \mu}, \alpha_{m}^{(2) \mu}$ and $\alpha_{-m}^{(2) \mu}$ through the prefactor $X_{\text {II }}^{2}$ or the vertex $|E\rangle$, which coincide with the sum of all the three amplitudes considered above on the string side in terms of the Neumann functions.

\subsection{Mixed impurities}

Next, we consider the case with one vector and one scalar impurities, namely, $i_{m} \mu_{-m}+$ vac $\rightarrow i_{n} \mu_{-n}$. The gauge theory result for $\left(\Delta_{2}+\Delta_{3}-\Delta_{1}\right) C_{123}$ is [14]

$$
\frac{\lambda^{\prime}}{2} C_{\mathrm{vac}} \frac{\sin ^{2}(\pi n y)}{y \pi^{2}\left(n^{2}-m^{2} / y^{2}\right)}\left(n+\frac{m}{y}\right)^{2} .
$$

On the string theory side, both the $X_{\mathrm{I}}$ and $X_{\mathrm{II}}$ parts in the prefactor in $\left|H_{3}\right\rangle_{h}$ contribute as

$$
\begin{aligned}
& { }_{123}\left\langle v\left|\alpha_{n}^{(1) i} \alpha_{-n}^{(1) \mu} \alpha_{m}^{(2) i} \alpha_{-m}^{(2) \nu} \frac{1}{2 \mu}\left(\sum_{i=5}^{8}\left(X_{\mathrm{I}}^{i}\right)^{2}+\sum_{\mu=1}^{4}\left(X_{\mathrm{II}}^{\mu}\right)^{2}\right)\right| E\right\rangle \\
& =\frac{1}{2 \mu}\left(\frac{\omega_{n}^{(1)}}{\alpha_{(1)}}+\frac{\omega_{m}^{(1)}}{\alpha_{(2)}}\right)\left(\widetilde{N}_{m-n}^{12}+\widetilde{N}_{m n}^{12}\right) \widetilde{N}_{m n}^{12}-\frac{1}{2 \mu}\left(\frac{\omega_{n}^{(1)}}{\alpha_{(1)}}+\frac{\omega_{m}^{(2)}}{\alpha_{(2)}}\right)\left(\widetilde{N}_{m-n}^{12}-\widetilde{N}_{m n}^{12}\right) \widetilde{N}_{m n}^{12},
\end{aligned}
$$

where the first part in the second line is the contribution from $\left(X_{\mathrm{I}}^{i}\right)^{2}|E\rangle$ and the second form $\left(X_{\mathrm{II}}^{\mu}\right)^{2}|E\rangle$, and the net result is

$$
\frac{1}{\mu}\left(\frac{\omega_{n}^{(1)}}{\alpha_{(1)}}+\frac{\omega_{m}^{(2)}}{\alpha_{(2)}}\right) \widetilde{N}_{m n}^{12} \widetilde{N}_{m n}^{12}
$$


We can easily confirm that in the large $\mu$ limit this precisely reduces to the field theory result (6.34).

\subsection{Fermionic impurities}

We first explain the convention for representing spinor impurities. We essentially follow the ref. 39]. Decompose the $\mathrm{SU}(4)$ R-symmetry group as $\mathrm{SU}(4)=\mathrm{SO}(4) \times \mathrm{U}(1)=$ $\mathrm{SU}(2) \times \mathrm{SU}(2) \times \mathrm{U}(1)$ with $U(1)$ being the subgroup corresponding to large orbital angular momentum $J$ :

$$
4 \rightarrow(2,1)_{+}+(1,2)_{-},
$$

where the subscript \pm represents the $\mathrm{U}(1)$ charge. With this decomposition of the Rcharge index, the correspondence between the fermionic fields $\lambda_{\alpha}^{A}$ and $\bar{\lambda}_{\dot{\alpha}}^{A}$, with $A$ and $\alpha$ being R-charge and Lorentz spinor indices, and the string theory creation operators $b_{\alpha_{1} \alpha_{2}}^{\dagger}$ and $b_{\dot{\alpha}_{1} \dot{\alpha}_{2}}^{\dagger}$ with $S O(8)(=S O(4) \times S O(4)=S U(2) \times S U(2) \times S U(2) \times S U(2))$ indices is given by

$$
\begin{aligned}
& \lambda_{\alpha}^{A} \rightarrow\left(\lambda_{r \alpha, 1 / 2}, \lambda_{\dot{r} \alpha,-1 / 2}\right) \sim\left(b_{\alpha_{1} \alpha_{2}}^{\dagger}, b_{\dot{\alpha}_{1} \alpha_{2}}^{\dagger}\right) \\
& \bar{\lambda}_{\dot{\alpha}}^{A} \rightarrow\left(\bar{\lambda}_{r \dot{\alpha},-1 / 2}, \bar{\lambda}_{\dot{r} \dot{\alpha}, 1 / 2}\right) \sim\left(b_{\alpha_{1} \dot{\alpha}_{2}}^{\dagger}, b_{\dot{\alpha}_{1} \dot{\alpha}_{2}}^{\dagger}\right)
\end{aligned}
$$

The original SU(4) index $A$ of $\lambda_{\alpha}^{A}$ is represented by the set $(r, 1 / 2)+(\dot{r},-1 / 2)$ and similarly

of $\bar{\lambda}_{\alpha}^{A}$ by its conjugate $(r,-1 / 2)+(\dot{r}, 1 / 2)$. Note that the range of indices are $r=3,4$, $\dot{r}=1,2, \alpha_{1}, \alpha_{2}=1,2$, and $\dot{\alpha}_{1}, \dot{\alpha}_{2}=1,2$. On the right end of the above symbolic relation, the fermion oscillators are represented by the indices $\left(r \sim \alpha_{1}, \alpha \sim \alpha_{2}\right)$ etc., with $\alpha$ being originally the spinor indices of $4 \mathrm{D}$ target space on the boundary. We call the sector with the $\mathrm{U}(1)$ charge $J=1 / 2$ a BMN fermion while the one with $J=-1 / 2$ an antiBMN fermion, and we will focus on the former. The $\mathrm{SU}(2)$ indices are contracted in the standard way by the $\epsilon$ symbol $\epsilon_{\alpha \beta}$ and $\epsilon^{\alpha \beta} \equiv \epsilon_{\alpha \beta}^{-1}$.

As for two fermionic impurities, it is sufficient to consider the four types listed bellow, accompanied with the corresponding gauge theory results for $\left(\Delta_{2}+\Delta_{3}-\Delta_{1}\right) C_{123}$ which can be extracted from the work [39]:

$$
\begin{array}{ll}
\lambda_{31 m} \lambda_{32-m}+\mathrm{vac} \rightarrow \lambda_{31 n} \lambda_{32-n}: & -\lambda^{\prime} C_{\mathrm{vac}} \frac{\sin ^{2}(\pi n y)}{y \pi^{2}} \frac{n}{n-m / y}, \\
\lambda_{31 m} \lambda_{31-m}+\mathrm{vac} \rightarrow \lambda_{31 n} \lambda_{31-n}: & -\lambda^{\prime} C_{\mathrm{vac}} \frac{\sin ^{2}(\pi n y)}{y \pi^{2}} \frac{2 n m / y}{n^{2}-m^{2} / y^{2}},
\end{array}
$$




$$
\begin{array}{ll}
\lambda_{31 m} \lambda_{42-m}+\operatorname{vac} \rightarrow \lambda_{31 n} \lambda_{42-n}: & -\frac{\lambda^{\prime}}{2} C_{\mathrm{vac}} \frac{\sin ^{2}(\pi n y)}{y \pi^{2}} \frac{n+m / y}{n-m / y}, \\
\lambda_{31 m} \lambda_{41-m}+\operatorname{vac} \rightarrow \lambda_{31 n} \lambda_{41-n}: & -\lambda^{\prime} C_{\mathrm{vac}} \frac{\sin ^{2}(\pi n y)}{y \pi^{2}} \frac{m / y}{n-m / y} .
\end{array}
$$

On the string theory side, the contribution from the $\left|H_{3}\right\rangle_{S V}$ part in the interaction vertex is already calculated in [39] as

$$
\begin{aligned}
\mu^{-1}\left\langle v\left|\beta_{-n}^{(1) 12} \beta_{n}^{(1) 11} \beta_{11, m}^{(2)} \beta_{12,-m}^{(2)}\right| H_{3}\right\rangle_{S V} & =-C_{N} \frac{\sin ^{2}(\pi n y)}{\left(\mu \alpha_{(1)}\right)^{2} \pi^{2} y} \\
\mu^{-1}\left\langle v\left|\beta_{-n}^{(1) 11} \beta_{n}^{(1) 11} \beta_{11, m}^{(2)} \beta_{11,-m}^{(2)}\right| H_{3}\right\rangle_{S V} & =0 \\
\mu^{-1}\left\langle v\left|\beta_{-n}^{(1) 22} \beta_{n}^{(1) 11} \beta_{11, m}^{(2)} \beta_{22,-m}^{(2)}\right| H_{3}\right\rangle_{S V} & =0 \\
\mu^{-1}\left\langle v\left|\beta_{-n}^{(1) 21} \beta_{n}^{(1) 11} \beta_{11, m}^{(2)} \beta_{21,-m}^{(2)}\right| H_{3}\right\rangle_{S V} & =C_{N} \frac{\sin ^{2}(\pi n y)}{\left(\mu \alpha_{(1)}\right)^{2} \pi^{2} y}
\end{aligned}
$$

where $\beta_{n}$ is the exponential basis of each string defined in a similar manner as $\alpha_{n}$ :

$$
\beta_{0}=b_{0}, \quad \beta_{n}=\frac{1}{\sqrt{2}}\left(b_{n}-i b_{-n}\right), \quad \beta_{-n}=\frac{1}{\sqrt{2}}\left(b_{n}+i b_{-n}\right) .
$$

For these cases of pure fermionic external states with undotted indices, the prefactor reduces to

$$
P_{S V}=\frac{\mu}{3} Y^{4}
$$

where $Y^{4}$ is defined as $Y^{4} \equiv Y_{\alpha_{1} \beta_{2}}^{2} Y^{2 \alpha_{1} \beta_{2}}=12 Y_{11} Y_{12} Y_{21} Y_{22}$ with $Y_{\alpha 1 \beta 1}^{2}=Y_{\alpha 1 \gamma_{2}} Y_{\beta 1}^{\gamma_{2}}$. See the Appendix B for the definitions of these quantities. Using the explicit form of $Y^{4}$, we can easily confirm that the amplitudes on the string side vanish in both case of (6.44) and (6.45), and that (6.46) is equal to the minus of (6.43). With the definition of $Y^{\alpha_{1} \alpha_{2}}$ in (B.44), the string-matrix element (6.43) is given by $-\left(\bar{G}_{n}^{(1)} \bar{G}_{m}^{(2)}\right)^{2}$, which reduces in the large $\mu$ limit to the right hand side of (6.43) using the asymptotic form for $\bar{G}^{(r)}$ given in (B.65).

Next turning to the contribution from the $\left|H_{3}\right\rangle_{D}$ part, we first find

$$
\begin{aligned}
\mu^{-1}\left\langle v\left|\beta_{-n}^{(1) 11} \beta_{n}^{(1) 11} \beta_{11, m}^{(2)} \beta_{11,-m}^{(2)}\right| H_{3}\right\rangle_{D} & =\left(\frac{2 \omega_{n}^{(1)}}{\mu \alpha_{(1)}}+\frac{2 \omega_{m}^{(2)}}{\mu \alpha_{(2)}}\right) \times \\
( & \left.-\frac{1}{4}\left(Q_{n m}^{12}+Q_{m n}^{21}\right)^{2}+\frac{1}{4}\left(Q_{n m}^{12}-Q_{m n}^{21}\right)^{2}\right) \\
& =-C_{N} \frac{\sin ^{2}(\pi n y)}{\left(\mu \alpha_{(1)}\right)^{2} y \pi^{2}} \frac{4 n m / y}{n^{2}-m^{2} / y^{2}}
\end{aligned}
$$

where we have used the relation $Q_{n m}^{12}=-N_{n m}^{12}, Q_{m n}^{21}=-N_{-m-n}^{21}$ in the large $\mu$ limit, which can be easily shown by the definition of $Q_{n m}^{r s},(\underline{\mathrm{B} .26})$, and the asymptotic form of 
$U^{(r)},($ B.65), given in the Appendix B. For other three cases (6.39), (6.41) and (6.42), we find that the $\left|H_{3}\right\rangle_{D}$ contribution gives one and the same expression

$$
\left(\frac{2 \omega_{n}^{(1)}}{\alpha_{(1)}}+\frac{2 \omega_{m}^{(2)}}{\alpha_{(2)}}\right) \frac{1}{4}\left(Q_{m n}^{21}-Q_{n m}^{12}\right)^{2}=-C_{N} \frac{\sin ^{2}(\pi n y)}{\left(\mu \alpha_{(1)}\right)^{2} y \pi^{2}} \frac{n+m / y}{n-m / y} .
$$

Combining these two types of contributions in each case, we obtain the matrix elements which precisely coincide with the gauge theory results (6.39)-(6.42).

\section{Concluding remarks}

Finally, we remark on some relevant problems left in the present paper and on possible future directions. First of all, we have to emphasize again that our main predictions are not restricted to the impurity-preserving processes which almost all of other works have been limited to. In the supergravity sector, our holographic relation summarized in (3.82) $\sim(3.84)$ is valid by its construction for general processes. The extremal cases of the supergravity sector where conformal dimensions are preserved $\Delta_{1}=\Delta_{2}+\Delta_{3}$ are generalized by lifting the degeneracy, owing to the higher-order effects in $\lambda^{\prime}$, to the impurity preserving processes including string excitation modes. In the last two sections, we have confirmed that our relation is indeed valid in this case with nontrivial stringy effects. It is quite plausible that the relation should then be naturally extended to impurity non-preserving sectors. We can note, for example, that the $\alpha^{\prime}$-correction factor $f$ in the holographic relation is consistent with the fact that the CFT coefficients for such cases start from 0 -th order in $1 / \mu$ just as the impurity-preserving processes, while the 3-point string vertices for such cases in general start at most from the first order in $1 / \mu$, because of the large $\mu$ behavior of the Neumann functions. In fact, it is easy to confirm that our ansatz gives the correct results for a few simple cases. However, a systematic check of more general classes of impurity non-preserving processes is beyond the scope of the present paper and is left to a forthcoming work.

There are many possible directions following the present work: For instance, in connection with the question of impurity nonpreserving processes, we should investigate the string-loop corrections. In most of the existing literature, impurity nonpreserving contributions have been ignored often without appropriate justification. It would also be interesting to extend the discussions of section 2 and 3 to higher-point correlation functions, from both standpoints of supergravity limit and full string theory, and to see to 
what extent the structure suggested in our first work [4] is realized in higher orders. Another important problem is to derive the holographic string-field theory in conjunction with our prescription of the holographic duality directly from the gauge-theory side. For such an attempt, the collective-field approach discussed in [40] seems to be suggestive.

\section{Acknowledgements}

We would like to thank H. Shimada for discussions at an early stage of the present work. The present work is supported in part by Grant-in-Aid for Scientific Research (No. 13135205 (Priority Areas) and No. 16340067 (B)) from the Ministry of Education, Science and Culture.

\section{A Reduction to $\mathrm{SO}(4)$ basis}

First we summarize the definitions of overlap integrals of $\mathrm{SO}(6)$ harmonics,

$$
Y^{I}=\mathcal{C}_{i_{1} \cdots i_{k}}^{I} x^{i_{1}} \cdots x^{i_{k}}
$$

following the convention of ref. [11]. Using the formula for the integration over the 5-dimensional unit sphere $S^{5}\left(\omega=\pi^{3}=\right.$ the area of a unit 5-sphere),

$$
\frac{1}{\omega_{5}} \int_{S^{5}} x^{i_{1}} \cdots x^{i_{2 m}}=\frac{2^{1-m}}{(m+2) !} \times(\text { All possiblle contractions }),
$$

we derive

$$
\begin{aligned}
& \int_{S^{5}} Y^{I_{1}} Y^{I_{2}}=\mathcal{C}_{i_{1} \cdots i_{k}}^{I_{1}} \mathcal{C}_{j_{1} \cdots j_{k}}^{I_{2}} \int_{S^{5}} x^{i_{1}} \cdots x^{i_{k}} x^{j_{1}} \cdots x^{j_{k}} \\
& =\pi^{3} \frac{2^{1-k}}{(k+2) !} k !\left\langle\mathcal{C}^{I_{1}} \mathcal{C}^{I_{2}}\right\rangle=\pi^{3} \frac{\delta^{I_{1} I_{2}}}{2^{k-1}(k+1)(k+2)}
\end{aligned}
$$

and

$$
\int_{S^{5}} Y^{I_{1}} Y^{I_{2}} Y^{I_{3}}=\pi^{3} \frac{2^{-(\Sigma-2) / 2}}{\left(\frac{\Sigma}{2}+2\right) !} \frac{k_{1} ! k_{2} ! k_{3} !}{\alpha_{1} ! \alpha_{2} ! \alpha_{3} !}\left\langle\mathcal{C}^{I_{1}} \mathcal{C}^{I_{2}} \mathcal{C}^{I_{3}}\right\rangle \equiv a\left(k_{1}, k_{2}, k_{3}\right)\left\langle\mathcal{C}^{I_{1}} \mathcal{C}^{I_{2}} \mathcal{C}^{I_{3}}\right\rangle
$$

since in this case $m=\Sigma / 2$ and $\frac{k_{1} ! k_{2} ! k_{3} !}{\alpha_{1} ! \alpha_{2} ! \alpha_{3} !}$ is equal to the number of same contractions occur, due to the total symmetry of the tensors $\mathcal{C}^{I}$.

Now we convert these integrals on $S^{5}$ into a Gaussian average for the $\mathrm{SO}(4)$ directions by taking the large J limit. Such a calculation has previously done in ref. [6] for some special cases. First by expressing the $S^{5}$ harmonics in terms the $S^{3}$ harmonics $\tilde{Y}^{I_{1}}$,

$$
Y^{I}=2^{-J / 2} \sqrt{\frac{(J+\tilde{k}) !}{J ! \tilde{k} !}} \mathrm{e}^{i J \psi} \cos ^{J} \theta \sin ^{\tilde{k}_{1}} \theta \tilde{Y}^{I_{1}}
$$




$$
Y^{\bar{I}}=2^{-J / 2} \sqrt{\frac{(J+\tilde{k}) !}{J ! \tilde{k} !}} \mathrm{e}^{-i J \psi} \cos ^{J} \theta \sin ^{\tilde{k}_{1}} \theta \tilde{Y}^{\bar{I}}
$$

Let us first check this formula by confirming the normalization integral.

$$
\int_{S^{5}} Y^{\bar{I}} Y^{I}=2^{-J} \frac{(J+\tilde{k}) !}{J ! \tilde{k} !} \int_{0}^{2 \pi} d \psi \int_{-\pi / 2}^{\pi / 2} d \theta \cos \theta|\sin \theta|^{3} \cos ^{2 J} \theta \sin ^{2 \tilde{k}} \theta \int_{S^{3}} \tilde{Y}^{\bar{I}} \tilde{Y}^{I}
$$

In the limit $J \rightarrow \infty$, this can be evaluated by making a change of integration variables from $\theta$ and the Cartesian coordinates $x^{i}$ on $S^{3}\left(\sum_{i=1}^{3}\left(x^{i}\right)^{2}=1\right)$ to a 4 -vector $y^{0}=\theta, y^{i}=\theta x^{i}$ as

$$
\begin{aligned}
\lim _{J \rightarrow \infty} \int_{S^{5}} Y^{\bar{I}} Y^{I} & =\lim _{J \rightarrow \infty} 2^{-J} \frac{(J+\tilde{k}) !}{J ! \tilde{k} !} 2 \pi \int d^{4} y \mathrm{e}^{-J y^{2}}\left|y^{\tilde{k}} \tilde{Y}^{I}\right|^{2} \\
& =2^{-J} \frac{(J+\tilde{k}) !}{J ! \tilde{k} !} 2 \pi \frac{\pi^{2}}{\left(J+\frac{1}{2}\right)^{2}} \frac{\tilde{k} !}{\left(2\left(J+\frac{1}{2}\right)\right)^{\tilde{k}}}\left\langle\tilde{\mathcal{C}}^{I} \tilde{\mathcal{C}}^{I}\right\rangle=\frac{\pi^{3}}{2^{J+\tilde{k}-1} J^{2}}
\end{aligned}
$$

which coincides with the (A.3.

Similarly, the 3-point integral is found to be

$$
\begin{gathered}
\int_{S^{5}} Y^{\bar{I}_{1}} Y^{I_{2}} Y^{I_{3}}=2^{-J_{1}}\left[\frac{\left(J_{1}+\tilde{k}_{1}\right) !\left(J_{2}+\tilde{k}_{2}\right) !\left(J_{3}+\tilde{k}_{3}\right) !}{J_{1} ! \tilde{k}_{1} ! J_{2} ! \tilde{k}_{2} ! J_{3} ! \tilde{k}_{3} !}\right]^{1 / 2} \\
\times 2 \pi \int d \theta \cos \theta|\sin \theta|^{3} \cos ^{J_{1}} \theta \sin ^{\tilde{k}_{1}} \theta \cos ^{J_{2}} \theta \sin ^{\tilde{k}_{2}} \theta \cos ^{J_{3}} \theta \sin ^{\tilde{k}_{3}} \theta \\
\rightarrow \pi^{3} \frac{1}{2^{-1+\frac{\Sigma}{2}} J_{1}^{2}}\left(\frac{J_{2}}{J_{1}}\right)^{\tilde{k}_{2} / 2}\left(\frac{J_{3}}{J_{1}}\right)^{\tilde{k}_{3} / 2} \frac{\sqrt{\tilde{k}_{1} ! \tilde{k}_{2} ! \tilde{k}_{3} !}}{\tilde{\alpha}_{1} ! \tilde{\alpha}_{2} ! \tilde{\alpha}_{3} !}\left\langle\tilde{\mathcal{C}}^{\bar{I}_{1}} \tilde{\mathcal{C}}^{I_{2}} \tilde{\mathcal{C}}^{I_{3}}\right\rangle .
\end{gathered}
$$

Equating this result with the large $J_{1}$ limit of (A.4) which is equal to

$$
\pi^{3} \frac{1}{2^{-1+\frac{\Sigma}{2}} J_{1}^{2}} \frac{1}{\tilde{\alpha}_{1} !}\left(\frac{J_{2} J_{3}}{J_{1}}\right)^{\tilde{\alpha}_{1}}\left\langle\mathcal{C}^{\bar{I}_{1}} \mathcal{C}^{I_{2}} \mathcal{C}^{I_{3}}\right\rangle
$$

we find the relation between the $\mathrm{SO}(6)$ and $\mathrm{SO}(4)$ contractions $\left(\tilde{\alpha}_{1}=\alpha_{1}=\left(\tilde{k}_{2}+\tilde{k}_{3}-\right.\right.$ $\left.\left.\tilde{k}_{1}\right) / 2\right)$,

$$
\left\langle\mathcal{C}^{\bar{I}_{1}} \mathcal{C}^{I_{2}} \mathcal{C}^{I_{3}}\right\rangle=\alpha_{1} !\left(\frac{J_{1}}{J_{2} J_{3}}\right)^{\tilde{\alpha}_{1}}\left(\frac{J_{2}}{J_{1}}\right)^{\tilde{k}_{2} / 2}\left(\frac{J_{3}}{J_{1}}\right)^{\tilde{k}_{3} / 2} \frac{\sqrt{\tilde{k}_{1} ! \tilde{k}_{2} ! \tilde{k}_{3} !}}{\tilde{\alpha}_{1} ! \tilde{\alpha}_{2} ! \tilde{\alpha}_{3} !}\left\langle\tilde{\mathcal{C}}^{\bar{I}_{1}} \tilde{\mathcal{C}}^{I_{2}} \tilde{\mathcal{C}}^{I_{3}}\right\rangle .
$$

This is used in section 3 in order to express the 3-point coupling in terms of the $\mathrm{SO}(4)$ variables explicitly.

\section{B Explicit form of string-field vertices}

In this appendix, we summarize various formulas which are necessary for our arguments in sections 5 and 6 . We hope that this is useful to make the expositions of the present paper self-contained. 


\section{B.1 Preliminaries}

First, the Fourier mode expansions in terms of $\sin / \cos$ basis of bosonic coordinate $x^{(r)}\left(\sigma_{r}\right)$ and bosonic momentum $p^{(r)}\left(\sigma_{r}\right)$, as well as the fermionic ones $\lambda^{(r)}\left(\sigma_{r}\right)$ and $\theta^{(r)}\left(\sigma_{r}\right)$, are given by

$$
\begin{aligned}
& x^{(r)}\left(\sigma_{r}\right)=x_{0}^{(r)}+\sqrt{2} \sum_{n=1}^{\infty}\left(x_{n}^{(r)} \cos \frac{n \sigma_{r}}{\left|\alpha_{(r)}\right|}+x_{-n}^{(r)} \sin \frac{n \sigma_{r}}{\left|\alpha_{(r)}\right|}\right) \\
& p^{(r)}\left(\sigma_{r}\right)=\frac{1}{2 \pi\left|\alpha_{(r)}\right|}\left[p_{0}^{(r)}+\sqrt{2} \sum_{n=1}^{\infty}\left(p_{n}^{(r)} \cos \frac{n \sigma_{r}}{\left|\alpha_{(r)}\right|}+p_{-n}^{(r)} \sin \frac{n \sigma_{r}}{\left|\alpha_{(r)}\right|}\right)\right] \\
& \theta^{(r)}\left(\sigma_{r}\right)=\theta_{0}^{(r)}+\sqrt{2} \sum_{n=1}^{\infty}\left(\theta_{n}^{(r)} \cos \frac{n \sigma_{r}}{\left|\alpha_{(r)}\right|}+\theta_{-n}^{(r)} \sin \frac{n \sigma_{r}}{\left|\alpha_{(r)}\right|}\right) \\
& \lambda^{(r)}\left(\sigma_{r}\right)=\frac{1}{2 \pi\left|\alpha_{(r)}\right|}\left[\lambda_{0}^{(r)}+\sqrt{2} \sum_{n=1}^{\infty}\left(\lambda_{n}^{(r)} \cos \frac{n \sigma_{r}}{\left|\alpha_{(r)}\right|}+\lambda_{-n}^{(r)} \sin \frac{n \sigma_{r}}{\left|\alpha_{(r)}\right|}\right)\right]
\end{aligned}
$$

where**

$$
\begin{aligned}
& x_{n}^{(r)}=\sqrt{\frac{\alpha^{\prime}}{2 \omega_{n}^{(r)}}}\left(a_{n}^{(r)}+a_{n}^{(r) \dagger}\right), \quad p_{n}^{(r)}=-i \sqrt{\frac{\omega_{n}^{(r)}}{2 \alpha^{\prime}}}\left(a_{n}^{(r)}-a_{n}^{(r) \dagger}\right), \\
& \theta_{0}^{(r)}=\frac{\sqrt{\alpha^{\prime}}}{2 \sqrt{\left|\alpha_{(r)}\right|}}\left[\frac{1+\Pi}{2}\left\{\left(1+e\left(\alpha_{(r)}\right)\right) b_{0}^{(r)}-\left(1-e\left(\alpha_{(r)}\right) b_{0}^{(r) \dagger}\right)\right\}\right. \\
& \left.+\frac{1-\Pi}{2}\left\{\left(1-e\left(\alpha_{(r)}\right)\right) b_{0}^{(r)}+\left(1+e\left(\alpha_{(r)}\right) b_{0}^{(r) \dagger}\right)\right\}\right], \\
& \theta_{n}^{(r)}=\frac{\sqrt{\alpha^{\prime}}}{\sqrt{2\left|\alpha_{(r)}\right|}} \sqrt{\frac{n}{\omega_{n}^{(r)}}}\left[\frac{1+\Pi}{2}\left\{U_{(r) n}^{-1 / 2} b_{n}^{(r)}+e\left(\alpha_{(r)} n\right) U_{(r) n}^{1 / 2} b_{-n}^{(r) \dagger}\right)\right\} \\
& \left.\left.+\frac{1-\Pi}{2}\left\{U_{(r) n}^{1 / 2} b_{n}^{(r)}+e\left(\alpha_{(r)} n\right) U_{(r) n}^{-1 / 2} b_{-n}^{(r) \dagger}\right)\right\}\right] \quad(n \neq 0) \\
& \lambda_{n}^{(r)}=\frac{\left|\alpha_{(r)}\right|}{\alpha^{\prime}} \theta_{n}^{(r) \dagger},
\end{aligned}
$$

with the ordinal (anti-)commutation relations

$$
\left[a_{m}^{(r) i}, a_{n}^{(s) j \dagger}\right]=\delta^{r s} \delta^{i j} \delta_{m n}, \quad\left\{b_{m}^{(r) a}, b_{n}^{(s) b}\right\}=\delta^{r s} \delta^{a b} \delta_{m n} .
$$

Here, $e(x) \equiv x /|x|, \omega_{n}^{(r)} \equiv \sqrt{n^{2}+\mu^{2} \alpha_{(r)}^{2}}, U_{(r) n} \equiv\left(\omega_{n}^{(r)}-\mu \alpha_{(r)}\right) /|n|$, and $\Pi \equiv \gamma^{1} \gamma^{2} \gamma^{3} \gamma^{4}$, with $\gamma^{i}$ being $\mathrm{SO}(8)$ gamma matrices. With these Fock space basis, the free string Hamiltonian for $r$-th string

$$
\left.H^{(r)}=\frac{1}{2} \int_{0}^{2 \pi\left|\alpha_{(r)}\right|} d \sigma\left[2 \pi \alpha^{\prime} p^{(r) 2}+\frac{1}{2 \pi \alpha^{\prime}}\left(\partial_{\sigma} x^{(r)}\right)^{2}+\frac{1}{2 \pi \alpha^{\prime}} \mu^{2} x^{(r) 2}\right)\right]
$$

** The definition of the oscillators $a_{ \pm n}^{(r)}$ is different from the usual one in the literature by a factor $-i$. We use this definition since it is the appropriate one in the supergravity limit as we have discussed in section 3 . 


$$
+\frac{1}{2} \int_{0}^{2 \pi\left|\alpha_{(r)}\right|} d \sigma\left[-2 \pi \alpha^{\prime} \lambda^{(r)} \partial_{\sigma} \lambda^{(r)}+\frac{1}{2 \pi \alpha^{\prime}} \theta^{(r)} \partial_{\sigma} \theta^{(r)}+2 \mu \lambda^{(r)} \Pi \theta^{(r)}\right]
$$

reduces to

$$
H=\sum_{n=-\infty}^{\infty} \frac{\omega_{n}^{(r)}}{\left|\alpha_{(r)}\right|}\left(a_{n}^{(r) \dagger} a_{n}^{(r)}+b_{n}^{(r) \dagger} b_{n}^{(r)}\right) .
$$

\section{B.2 Overlap vertex}

The overlap vertex takes the form

$$
|E\rangle=\left|E_{a}\right\rangle\left|E_{b}\right\rangle \delta\left(\sum_{r=1}^{3} \alpha_{(r)}\right),
$$

where $\left|E_{a}\right\rangle$ and $\left|E_{b}\right\rangle$ are the bosonic and fermionic overlap vertices which satisfy

$$
\begin{aligned}
& \sum_{r=1}^{3} \tilde{p}^{(r)}(\sigma)\left|E_{a}\right\rangle=0, \quad \sum_{r=1}^{3} e\left(\alpha_{(r)}\right) \tilde{x}^{(r)}(\sigma)\left|E_{a}\right\rangle=0, \\
& \sum_{r=1}^{3} \tilde{\lambda}^{(r)}(\sigma)\left|E_{b}\right\rangle=0, \quad \sum_{r=1}^{3} e\left(\alpha_{(r)}\right) \tilde{\theta}^{(r)}(\sigma)\left|E_{b}\right\rangle=0 .
\end{aligned}
$$

Here, $\tilde{p}^{(r)}(\sigma)\left(|\sigma| \leq\left|\alpha_{(1)}\right|\right)$ is defined as $\tilde{p}^{(r)}(\sigma) \equiv \Theta_{r}(\sigma) p^{(r)}\left(\sigma_{r}\right)$ with $\Theta_{2}(\sigma)=\theta\left(\pi \alpha_{(2)}-|\sigma|\right)$, $\Theta_{3}(\sigma)=\theta\left(|\sigma|-\pi \alpha_{(2)}\right)$, and $\Theta_{1}=1$. The parameter $\sigma_{r}$ is defined as

$$
\begin{array}{ll}
\sigma_{2}=\sigma & -\pi \alpha_{(2)} \leq \sigma \leq \pi \alpha_{(2)} \\
\sigma_{3}= \begin{cases}\sigma-\pi \alpha_{(2)} & \pi \alpha_{(2)} \leq \sigma \leq \pi\left(\alpha_{(2)}+\alpha_{(3)}\right) \\
\sigma+\pi \alpha_{(2)} & -\pi\left(\alpha_{(2)}+\alpha_{(3)}\right) \leq \sigma \leq-\pi \alpha_{(2)}\end{cases} \\
\sigma_{1}=-\sigma & -\pi\left(\alpha_{(2)}+\alpha_{(3)}\right) \leq \sigma \leq \pi\left(\alpha_{(2)}+\alpha_{(3)}\right) .
\end{array}
$$

The definitions of $\tilde{x}_{(r)}(\sigma), \tilde{\lambda}_{(r)}(\sigma)$ and $\tilde{\theta}_{(r)}(\sigma)$ are given in the same way. We always assume $\alpha_{(r)}\left(\equiv \alpha^{\prime} p_{(r)}^{+}\right)$satisfies the relation $\alpha_{(2)}, \alpha_{(3)}>0, \alpha_{(1)}<0$.

The explicit form of the overlap vertex is

$$
\begin{aligned}
& \left|E_{a}\right\rangle=\exp \left[-\frac{1}{2} \sum_{r, s=1}^{3} a_{m}^{(r) \dagger} N_{m n}^{r s} a_{n}^{(s) \dagger}\right]\left|v_{a}\right\rangle_{123} \\
& \left|E_{b}\right\rangle=\exp \left[\sum_{r, s=1}^{3} \sum_{m, n=0}^{\infty}\left(b_{-m}^{(r) \alpha_{1} \alpha_{2} \dagger} Q_{m n}^{r s} b_{n \alpha_{1} \alpha_{2}}^{(s) \dagger}+b_{m}^{(r) \dot{\alpha}_{1} \dot{\alpha}_{2} \dagger} Q_{m n}^{r s} b_{-n \dot{\alpha}_{1} \dot{\alpha}_{2}}^{(s) \dagger}\right)\right]\left|v_{b}\right\rangle_{123} .
\end{aligned}
$$

This overlap vertex is based on the ground states $\left|v_{a}\right\rangle_{123}$ and $\left|v_{b}\right\rangle_{123}$ which are defined by $a_{n}^{(r)}\left|v_{a}\right\rangle_{123}=0$ and $b_{n}^{(r)}\left|v_{b}\right\rangle_{123}=0$ for $n \in Z$. Note that $\left|E_{b}\right\rangle$ is constructed on the Fock vacuum $\left|v_{b}\right\rangle$ [34, 35, not on the $\mathrm{SO}(8)$ vacuum $|0\rangle$, defined as $a_{n}^{(r)}|0\rangle=0(n \in Z)$, 
$b_{n}^{(r)}|0\rangle=0(n \neq 0)$ and $\theta_{0}^{(r)}|0\rangle=0$, on which the original fermionic interaction vertex [17, 18, 19] was constructed. As for the fermionic sector, the $\mathrm{SO}(8)$ spinor indices have been decomposed as $\mathrm{SO}(8)=\mathrm{SO}(4) \times \mathrm{SO}(4)=\mathrm{SU}(2) \times \mathrm{SU}(2) \times \mathrm{SU}(2) \times \mathrm{SU}(2)$, according to the works [20, 21].

The bosonic Neumann coefficients $N_{m n}^{r s}, N_{m}^{r}$ and the fermionic Neumann coefficients $Q_{m n}^{r s}, Q_{m}^{r}$ are given by

$$
\begin{aligned}
& N_{00}^{r^{\prime} s^{\prime}}=(1-4 \mu \alpha K)\left(\delta^{r^{\prime} s^{\prime}}+\frac{\sqrt{\alpha_{\left(r^{\prime}\right)} \alpha_{\left(s^{\prime}\right)}}}{\alpha_{(1)}}\right), \\
& N_{00}^{r^{\prime} 1}=\delta^{r^{\prime} 1}-\sqrt{-\frac{\alpha_{\left(r^{\prime}\right)}}{\alpha_{(1)}}} \\
& N_{m 0}^{r s^{\prime}}=-\sqrt{2 \mu \alpha_{\left(s^{\prime}\right)}} \epsilon_{s^{\prime} t^{\prime}} \alpha_{\left(t^{\prime}\right)}\left(C_{(r)}^{1 / 2} N^{r}\right)_{m}, \\
& N_{m}^{r}=-\left(C^{-1 / 2} A^{(r) \mathrm{T}} \Gamma^{-1} B\right)_{m}, \\
& N_{m n}^{r s}=\delta^{r s} \delta_{m n}-2\left(C_{(r)}^{1 / 2} C^{-1 / 2} A^{(r) \mathrm{T}} \Gamma^{-1} A^{(s)} C^{-1 / 2} C_{(s)}^{1 / 2}\right)_{m n}, \\
& N_{-m-n}^{r s}=-\left(U_{(r)} N^{r s} U_{(s)}\right)_{m n}, \\
& Q_{m n}^{r s}=e\left(\alpha_{(r)}\right) \sqrt{\frac{\left|\alpha_{(s)}\right|}{\left|\alpha_{(r)}\right|}}\left(U_{(r)}^{1 / 2} C^{1 / 2} N^{r s} C^{-1 / 2} U_{(s)}^{1 / 2}\right)_{m n}, \\
& Q_{m 0}^{r r^{\prime}}=-\epsilon_{r^{\prime} t^{\prime}} \sqrt{\alpha_{\left(r^{\prime}\right)}} \alpha_{\left(t^{\prime}\right)} \frac{e\left(\alpha_{(r)}\right)}{\sqrt{\left|\alpha_{(r)}\right|}}\left(U_{(r)}^{1 / 2} C_{(r)}^{1 / 2} C^{1 / 2} N^{r}\right)_{m}, \\
& Q_{00}^{1 r^{\prime}}=-Q_{00}^{r^{\prime} 1}=\frac{1}{2} \sqrt{-\frac{\alpha_{\left(r^{\prime}\right)}}{\alpha_{(1)}}} \\
& \text { Otherwise }=0 \text {, }
\end{aligned}
$$

where $n, m>0, r^{\prime}, s^{\prime} \in\{2,3\}, r, s \in\{1,2,3\}, \alpha \equiv \alpha_{(1)} \alpha_{(2)} \alpha_{(3)}$, and

$$
\begin{aligned}
& C_{m n}=m \delta_{m n}, \quad C_{(r) m n}=\omega_{m(r)} \delta_{m n}, \quad U_{(r)}=C^{-1}\left(C_{(r)}-\mu \alpha_{(r)}\right), \\
& K=-\frac{1}{4} B^{\mathrm{T}} \Gamma^{-1} B, \quad \Gamma=\sum_{r=1}^{3} A^{(r)} U_{(r)} A^{(r) \mathrm{T}} \\
& A_{m n}^{(2)}=\frac{2 \sqrt{m n}}{\pi} \frac{y(-1)^{n+1}}{n^{2}-y^{2} m^{2}} \sin (\pi m y), \quad A_{m n}^{(3)}=\frac{2 \sqrt{m n}}{\pi} \frac{(1-y)}{n^{2}-(1-y)^{2} m^{2}} \sin \pi m y, \\
& A_{m n}^{(1)}=\delta_{m n}, \quad B_{m}=\frac{2}{\pi y(1-y) \alpha_{(1)}} m^{-3 / 2} \sin (\pi m y),
\end{aligned}
$$

with $y=-\alpha_{(2)} / \alpha_{(1)}$ and $1-y=-\alpha_{(3)} / \alpha_{(1)}$.

When we compare string amplitudes on the both sides of bulk and boundary in the plane-wave limit, the appropriate Fock basis is the one spanned by the oscillators defined 
with the exponential Fourier mode basis which corresponds directly to BMN operators:

$$
\alpha_{0}=a_{0}, \quad \alpha_{n}=\frac{1}{\sqrt{2}}\left(a_{n}-i a_{-n}\right), \quad \alpha_{-n}=\frac{1}{\sqrt{2}}\left(a_{n}+i a_{-n}\right) .
$$

The Neumann coefficients in terms of the exponential oscillator basis, $\widetilde{N}_{m n}^{r s}$, is

$$
\sum_{m, n=-\infty}^{\infty} a_{m}^{(r) \dagger} N_{m n}^{r s} a_{n}^{(s) \dagger}=\sum_{m, n=-\infty}^{\infty} \alpha_{m}^{(r) \dagger} \widetilde{N}_{m n}^{r s} \alpha_{n}^{(s) \dagger},
$$

where

$$
\begin{aligned}
& \widetilde{N}_{00}^{r s}=N_{00}^{r s}, \quad \widetilde{N}_{0 m}^{r s}=\widetilde{N}_{m 0}^{r s}=\widetilde{N}_{0-m}^{r s}=\widetilde{N}_{-m 0}^{r s}=\frac{1}{\sqrt{2}} N_{0 m}^{r s} \\
& \widetilde{N}_{m n}^{r s}=\widetilde{N}_{-m-n}^{r s}=\frac{1}{2}\left(N_{m n}^{r s}-N_{-m-n}^{r s}\right), \quad \widetilde{N}_{m-n}^{r s}=\widetilde{N}_{-m n}^{r s}=\frac{1}{2}\left(N_{m n}^{r s}+N_{-m-n}^{r s}\right)
\end{aligned}
$$

We present the large $\mu$ behavior of $\widetilde{N}_{m n}^{r s}$ 's below in the Appendix B.4.

\section{B.3 Prefactors}

The prefactor which was first constructed for the overlap vertex based on the $\mathrm{SO}(8)$ vacuum $|0\rangle$ [16, 17, 18, 19] can be reformulated for the overlap vertex $|E\rangle$ which is based on the genuine Fock vacuum [20, 21]. The form of this prefactor is ${ }^{\dagger \dagger}$

$$
\begin{aligned}
& \left|H_{3}\right\rangle_{S V}=P_{S V}|E\rangle, \\
& P_{S V}=\frac{1}{2}\left[\left(K^{i} \widetilde{K}^{j}+\mu \delta^{i j}\right) V_{i j}-\left(K^{\mu} \widetilde{K}^{\nu}+\mu \delta^{\mu \nu}\right) V_{\mu \nu}\right. \\
& \left.\quad-K^{\dot{\alpha}_{1} \alpha_{1}} \widetilde{K}^{\dot{\alpha}_{2} \alpha_{2}} S_{\alpha_{1} \alpha_{2}}(Y) S_{\dot{\alpha}_{1} \dot{\alpha}_{2}}^{*}(Z)-\widetilde{K}^{\dot{\alpha}_{1} \alpha_{1}} K^{\dot{\alpha}_{2} \alpha_{2}} S_{\alpha_{1} \alpha_{2}}^{*}(Y) S_{\dot{\alpha}_{1} \dot{\alpha}_{2}}(Z)\right]
\end{aligned}
$$

where $K^{I}, \widetilde{K}^{I}(I=i, \mu)$ and $Y^{\alpha_{1} \alpha_{2}}, Z^{\dot{\alpha}_{1} \dot{\alpha}_{2}}$ are bosonic and fermionic constituents of the prefactor defined as

$$
\begin{aligned}
& K^{J}=X_{\mathrm{I}}^{J}+X_{\mathrm{II}}^{J}, \quad \widetilde{K}^{J}=X_{\mathrm{I}}^{J}-X_{\mathrm{II}}^{J}, \\
& X_{\mathrm{I}}=i \sqrt{-\frac{\alpha^{\prime}}{\alpha}}(1-4 \mu \alpha K)^{1 / 2} \sum_{r=1}^{3} \sum_{n=0}^{\infty} F_{n}^{(r)} a_{n}^{(r) \dagger}, \\
& X_{\mathrm{II}}=-\sqrt{-\frac{\alpha^{\prime}}{\alpha}}(1-4 \mu \alpha K)^{1 / 2} \sum_{r=1}^{3} \sum_{n=1}^{\infty} U_{n(r)} F_{n}^{(r)} a_{-n}^{(r) \dagger}, \\
& Y^{\alpha_{1} \alpha_{2}}=\sqrt{-\frac{\alpha^{\prime}}{\alpha}}(1-4 \mu \alpha K)^{1 / 2} \sum_{r=1}^{3} \sum_{n=0}^{\infty} G_{n}^{(r)} b_{n}^{(r) \alpha_{1} \alpha_{2} \dagger}, \\
& Z^{\dot{\alpha}_{1} \dot{\alpha}_{2}}=\sqrt{-\frac{\alpha^{\prime}}{\alpha}}(1-4 \mu \alpha K)^{1 / 2} \sum_{r=1}^{3} \sum_{n=0}^{\infty} G_{n}^{(r)} b_{-n}^{(r) \dot{\alpha}_{1} \dot{\alpha}_{2} \dagger},
\end{aligned}
$$

${ }^{\dagger \dagger}$ This definition differs form the one in (3.28) of [20] by a factor $-2 \alpha / \alpha^{\prime}$. Note also the difference of the total factor of $K^{i}$ between here and there. 
with

$$
\begin{aligned}
& F_{0}^{(2)}=-\sqrt{\frac{2}{\alpha^{\prime}}} \sqrt{\mu \alpha_{(2)}} \alpha_{(3)}, \quad F_{0}^{(3)}=\sqrt{\frac{2}{\alpha^{\prime}} \sqrt{\mu \alpha_{(3)}} \alpha_{(2)}, \quad F_{0}^{(1)}=0,} \\
& F_{n}^{(r)}=-\frac{\alpha}{\sqrt{\alpha^{\prime}}} \frac{1}{1-4 \mu \alpha K} \frac{1}{\alpha_{(r)}}\left(U_{(r)}^{-1} C_{(r)}^{1 / 2} C N^{r}\right)_{n} \quad(n>0), \\
& G_{0}^{(2)}=-\sqrt{\frac{1}{\alpha^{\prime}}} \sqrt{\alpha_{(2)}} \alpha_{(3)}, \quad G_{0}^{(3)}=\sqrt{\frac{1}{\alpha^{\prime}}} \sqrt{\alpha_{(3)}} \alpha_{(2)}, \quad G_{0}^{(1)}=0, \\
& G_{n}^{(r)}=-\frac{\alpha}{\sqrt{\alpha^{\prime}}} \frac{1}{1-4 \mu \alpha K} \frac{e\left(\alpha_{(r)}\right)}{\sqrt{\left|\alpha_{(r)}\right|}}\left(U_{(r)}^{-1 / 2} C_{(r)}^{1 / 2} C^{1 / 2} N^{r}\right)_{n} \quad(n>0) .
\end{aligned}
$$

The other quantities in the prefactor is defined as

$$
\begin{aligned}
& \begin{aligned}
& V_{i j} \equiv \delta_{i j}\left[1+\frac{1}{12}\left(Y^{4}+\right.\right.\left.\left.Z^{4}\right)+\frac{1}{144} Y^{4} Z^{4}\right] \\
&-\frac{i}{2}\left[Y_{i j}^{2}\left(1+Z^{4}\right)-Z_{i j}^{2}\left(1+\frac{1}{12} Y^{4}\right)\right]+\frac{1}{4}\left(Y^{2} Z^{2}\right)_{i j}, \\
& V_{\mu \nu} \equiv \delta_{\mu \nu}\left[1-\frac{1}{12}\left(Y^{4}+Z^{4}\right)+\frac{1}{144} Y^{4} Z^{4}\right] \\
& \quad-\frac{i}{2}\left[Y_{\mu \nu}^{2}\left(1-Z^{4}\right)-Z_{\mu \nu}^{2}\left(1-\frac{1}{12} Y^{4}\right)\right]+\frac{1}{4}\left(Y^{2} Z^{2}\right)_{\mu \nu}
\end{aligned} \\
& S(Y) \equiv Y+\frac{i}{3} Y^{3},
\end{aligned}
$$

with

$$
\begin{aligned}
& K^{\dot{\alpha}_{r} \beta_{r}} \equiv K^{i} \sigma_{i}^{\dot{\alpha}_{r} \beta_{r}}, \quad \widetilde{K}^{\dot{\alpha}_{r} \beta_{r}} \equiv K^{i} \sigma_{i}^{\dot{\alpha}_{r} \beta_{r}}, \quad(r=1,2) \\
& Y_{\alpha_{1} \beta_{1}}^{2} \equiv Y_{\alpha_{1} \alpha_{2}} Y_{\beta_{1}}^{\alpha_{2}}, \quad Y_{\alpha_{2} \beta_{2}}^{2} \equiv Y_{\alpha_{1} \alpha_{2}} Y_{\beta_{2}}^{\alpha_{1}}, \\
& Y_{\alpha_{1} \beta_{2}}^{3} \equiv Y_{\alpha_{1} \beta_{1}}^{2} Y_{\beta_{2}}^{\beta_{1}}, \quad Y^{4} \equiv Y_{\alpha_{1} \beta_{1}}^{2} Y^{2 \alpha_{1} \beta_{1}}, \\
& Y^{2 i j} \equiv Y^{2 \alpha_{1} \beta_{1}} \sigma_{\alpha_{1} \beta_{1}}^{i j}, \quad Z^{2 i j} \equiv Z^{2 \dot{\alpha}_{1} \dot{\beta}_{1}} \sigma_{\dot{\alpha}_{1} \dot{\beta}_{1}}^{i j}, \quad\left(Y^{2} Z^{2}\right)^{i j} \equiv Y^{2 k(i} Z^{2 j) k},
\end{aligned}
$$

We refer the reader to the ref.[20] for more details.

On the other hand, the interaction vertex presented in [26], which is of the form

$$
\left|H_{3}\right\rangle_{D}=\sum_{r=1}^{3} \sum_{m=-\infty}^{\infty} \frac{\omega_{m}^{(r)}}{\alpha_{(r)}}\left(\sum_{I=1}^{8} a_{m}^{(r) I \dagger} a_{m}^{(r) I}+\sum_{a=1}^{8} b_{m}^{(r) a \dagger} b_{m}^{(r) a}\right)|E\rangle
$$

can be, using the factorization formula, written as

$$
\begin{aligned}
& \left|H_{3}\right\rangle_{D}=P_{D}|E\rangle \\
& P_{D}=\frac{1}{4}\left(K^{2}+\widetilde{K}^{2}\right)-Y^{\alpha_{1} \alpha_{2}} \widetilde{Y}_{\alpha_{1} \alpha_{2}}-Z^{\dot{\alpha}_{1} \dot{\alpha}_{2}} \widetilde{Z}_{\dot{\alpha}_{1} \dot{\alpha}_{2}}
\end{aligned}
$$


where

$$
\tilde{Y}^{\alpha_{1} \alpha_{2}}=\sum_{r=1}^{3} \frac{n}{\alpha_{(r)}} G_{n}^{(r)} b_{n}^{(r) \alpha_{1} \alpha_{2} \dagger}, \quad \widetilde{Z}^{\dot{\alpha}_{1} \dot{\alpha}_{2}}=\sum_{r=1}^{3} \frac{n}{\alpha_{(r)}} G_{n}^{(r)} b_{-n}^{(r) \alpha_{1} \alpha_{2} \dagger} .
$$

For the derivation of (B.57), see the Appendix B.5 below.

\section{B.4 Large $\mu$ behavior $[25]$}

The large $\mu$ behavior of $\widetilde{N}_{m n}^{r s}$ is given, for $(m, n) \neq(0,0)$, by

$$
\begin{aligned}
& \widetilde{N}_{m n}^{22}=\frac{(-1)^{m+n}}{4 \pi \mu\left|\alpha_{(1)}\right| y}, \quad \widetilde{N}_{m n}^{23}=\frac{(-1)^{m+1}}{4 \pi \mu\left|\alpha_{(1)}\right| \sqrt{y(1-y)}} \\
& \widetilde{N}_{m n}^{33}=\frac{1}{4 \pi \mu\left|\alpha_{(1)}\right|(1-y)}, \quad \widetilde{N}_{m n}^{11}=\frac{(-1)^{m+n+1} \sin (\pi m y) \sin (\pi n y)}{\pi \mu\left|\alpha_{(1)}\right|} \\
& \widetilde{N}_{m n}^{21}=\frac{(-1)^{m+n+1} \sin (\pi n y)}{\pi \sqrt{y}(n-m / y)}, \quad \widetilde{N}_{m n}^{31}=\frac{(-1)^{n} \sin (\pi n y)}{\pi \sqrt{1-y}(n-m /(1-y))}
\end{aligned}
$$

and, for $m=n=0$, by

$$
\begin{aligned}
& \widetilde{N}_{00}^{11}=0, \quad \widetilde{N}_{00}^{12}=-\sqrt{y}, \quad \widetilde{N}_{00}^{13}=-\sqrt{1-y} \\
& \widetilde{N}_{00}^{23}=-\frac{1}{4 \pi \mu\left|\alpha_{(1)}\right| \sqrt{y(1-y)}}, \quad \widetilde{N}_{00}^{22}=\frac{1}{4 \pi \mu\left|\alpha_{(1)}\right| y}, \quad \widetilde{N}_{00}^{33}=\frac{1}{4 \pi \mu\left|\alpha_{(1)}\right|(1-y)}
\end{aligned}
$$

When we compute string amplitudes for fermions, the large $\mu$ behavior of $F_{n}^{(r)}, G_{n}^{(r)}$, $U_{m}^{(r)}$ and $1-4 \mu \alpha K$ are also useful ${ }^{\ddagger \ddagger}$ :

$$
\begin{aligned}
& F_{n}^{(2)}=(-1)^{n+1} \frac{2\left|\alpha_{(1)}\right|}{\sqrt{\alpha^{\prime}}} \sqrt{\mu\left|\alpha_{(1)}\right| y}(1-y), \quad F_{n}^{(3)}=\frac{2\left|\alpha_{(1)}\right|}{\sqrt{\alpha^{\prime}}} \sqrt{\mu\left|\alpha_{(1)}\right|(1-y)} y, \\
& F_{n}^{(1)}=(-1)^{n+1} \frac{2\left|\alpha_{(1)}\right|}{\sqrt{\alpha^{\prime}}} \frac{y(1-y)}{\sqrt{\mu\left|\alpha_{(1)}\right|}} n \sin (\pi n y), \\
& \bar{G}_{n}^{(2)}=\frac{(-1)^{n+1}}{\sqrt{2 \pi \mu\left|\alpha_{(1)}\right| y}}, \quad \bar{G}_{n}^{(3)}=\frac{1}{\sqrt{2 \pi \mu\left|\alpha_{(1)}\right|(1-y)}}, \quad \bar{G}_{n}^{(1)}=\frac{(-1)^{n+1} \sqrt{2} \sin (\pi n y)}{\sqrt{\pi \mu\left|\alpha_{(1)}\right|}} \\
& U_{n}^{(2)}=\frac{n}{2 \mu\left|\alpha_{(1)}\right| y}, \quad U_{n}^{(3)}=\frac{n}{2 \mu\left|\alpha_{(1)}\right|(1-y)}, \quad U_{n}^{(1)}=\frac{2 \mu\left|\alpha_{(1)}\right|}{n} \\
& 1-4 \mu \alpha K=\frac{1}{4 \pi \mu\left|\alpha_{(1)}\right| y(1-y)}
\end{aligned}
$$

where we have defined

$$
\bar{G}_{n}^{(r)}=\sqrt{-\frac{\alpha^{\prime}}{\alpha}}(1-4 \mu \alpha K)^{1 / 2} G_{n}^{(r)} .
$$

$\ddagger_{\ddagger}$ Note that the definition of the Neumann vector $N_{n}^{r}$ here, with which $F_{n}^{(r)}$ and $G_{n}^{(r)}$ are defined, differs by $C_{(r)}^{-1 / 2} U_{r}$ from that of the ref. [25]. 


\section{B.5 Factorization formula}

We first prove the formula

$$
\sum_{r=1}^{3} \sum_{n=0}^{\infty} \frac{\omega_{n}^{(r)}}{\alpha_{(r)}} a_{n}^{(r) \dagger} a_{n}^{(r)}|E\rangle=\frac{1}{2} X_{\mathrm{I}}^{2}|E\rangle
$$

using the factorization formula obtained in [23, 18]. Operating the annihilation operator $a_{n}$ on the vertex $|E\rangle$, the left hand side of (B.67) can be written as

$$
\begin{aligned}
\sum_{r=1}^{3} \sum_{n=0}^{\infty} \frac{\omega_{n}^{(r)}}{\alpha_{(r)}} a_{n}^{(r) \dagger} a_{n}^{(r)}|E\rangle= & -\sum_{r, s=1}^{3}\left(\frac{\omega_{0}^{(r)}}{\alpha_{(r)}} N_{00}^{r s} a_{0}^{(r) \dagger} a_{0}^{(s) \dagger}+\sum_{n=1}^{\infty} \frac{\omega_{0}^{(r)}}{\alpha_{(r)}} N_{0 n}^{r s} a_{0}^{(r) \dagger} a_{n}^{(s) \dagger}\right. \\
& \left.+\sum_{n=1}^{\infty} \frac{\omega_{n}^{(r)}}{\alpha_{(r)}} N_{n 0}^{r s} a_{n}^{(r) \dagger} a_{0}^{(s) \dagger}+\sum_{n, m=1}^{\infty} \frac{\omega_{m}^{(r)}}{\alpha_{(r)}} N_{m n}^{r s} a_{m}^{(r) \dagger} a_{n}^{(s) \dagger}\right)|E\rangle .
\end{aligned}
$$

By the definition of Neumann matrices, the first term in the right hand side becomes

$$
\sum_{r, s=1}^{3} \frac{\omega_{0}^{(r)}}{\alpha_{(r)}} N_{00}^{r s} a_{0}^{(r) \dagger} a_{0}^{(s) \dagger}=\frac{1}{2} X_{0}^{2}
$$

and the sum of the second and the third terms reduces to

$$
\sum_{r, s=1}^{3} \sum_{n=1}^{\infty} \frac{\omega_{0}^{(r)}}{\alpha_{(r)}} N_{0 n}^{r s} a_{0}^{(r) \dagger} a_{n}^{(s) \dagger}+\sum_{r, s=1}^{3} \sum_{n=1}^{\infty} \frac{\omega_{n}^{(r)}}{\alpha_{(r)}} N_{n 0}^{r s} a_{n}^{(r) \dagger} a_{0}^{(s) \dagger}=X_{0} X_{+},
$$

where $X_{0}$ and $X_{+}$is defined as zero-mode and positive-mode parts of $X_{\mathrm{I}}$, such as

$$
X_{\mathrm{I}}=i \sqrt{-\frac{\alpha^{\prime}}{\alpha}}(1-4 \mu \alpha K)^{1 / 2}\left(\sum_{r=1}^{3} F_{0}^{(r)} a_{0}^{(r) \dagger}+\sum_{r=1}^{3} \sum_{n=1}^{\infty} F_{n}^{(r)} a_{n}^{(r) \dagger}\right) \equiv X_{0}+X_{+} .
$$

Using the property of the Neumann matrix, $N_{n m}^{r s}=N_{m n}^{s r}$, and the factorization formula [23, 18],

$$
N_{n m}^{r s}=-\frac{\alpha}{1-4 \mu \alpha K} \frac{1}{\alpha_{(r)} \omega_{m}^{(s)}+\alpha_{(s)} \omega_{n}^{(r)}}\left(U_{(r)}^{-1} C_{(r)}^{1 / 2} C N^{r}\right)_{n}\left(U_{(r)}^{-1} C_{(s)}^{1 / 2} C N^{s}\right)_{m},
$$

the fourth term can be written as

$$
\begin{aligned}
\sum_{r, s=1}^{3} \sum_{n, m=1}^{\infty} \frac{\omega_{n}^{(r)}}{\alpha_{(r)}} N_{n m}^{r s} a_{n}^{(r) \dagger} a_{m}^{(s) \dagger} & =\frac{1}{2} \sum_{r, s=1}^{3} \sum_{n, m=1}^{\infty}\left(\frac{\omega_{n}^{(r)}}{\alpha_{(r)}} N_{n m}^{r s}+N_{n m}^{r s} \frac{\omega_{m}^{(s)}}{\alpha_{(s)}}\right) a_{n}^{(r) \dagger} a_{m}^{(s) \dagger} \\
& =\frac{1}{2} X_{+}^{2} .
\end{aligned}
$$

Combining all the results above, we obtain the formula (B.67). 
Noticing that the Neumann coefficient with negative Fourier modes is given by

$$
N_{-m-n}^{r s}=-\left(U_{(r)} N^{r s} U_{(s)}\right)_{m n}, \quad(m, n>0)
$$

and the definition of $X_{\mathrm{II}}$, which has the extra $i U_{(r)}$ factor compared with $X_{\mathrm{I}}$, we can easily see that the similar formula for negative modes,

$$
\sum_{r=1}^{3} \sum_{n=1}^{\infty} \frac{\omega_{n}^{(r)}}{\alpha_{(r)}} a_{-n}^{(r) \dagger} a_{-n}^{(r)}|E\rangle=\frac{1}{2} X_{\mathrm{II}}^{2}|E\rangle,
$$

can also hold.

With the definition of the fermionic Neumann coefficient $Q_{m n}^{r s}$, it is not difficult to prove the formula

$$
\begin{aligned}
\sum_{r=1}^{3} \sum_{n \in Z} \frac{\omega_{n}^{(r)}}{\alpha_{(r)}}\left(b_{n \alpha_{1} \alpha_{2}}^{(r) \dagger} b_{n}^{(r) \alpha_{1} \alpha_{2}}+b_{n \dot{\alpha} \dot{\alpha}_{2}}^{(r) \dagger} b_{n}^{(r) \dot{\alpha}_{1} \dot{\alpha}_{2}}\right)\left|E_{b}\right\rangle \\
=-\left(Y_{\alpha_{1} \alpha_{2}} \tilde{Y}^{\alpha_{1} \alpha_{2}}+Z_{\dot{\alpha}_{1} \dot{\alpha}_{2}} \widetilde{Z}^{\dot{\alpha}_{1} \dot{\alpha}_{2}}\right)\left|E_{b}\right\rangle
\end{aligned}
$$

in the same manner as the bosonic case.

\section{References}

[1] D. Berenstein, J. M. Maldacena, H. Nastase, Strings in flat space and $p p$ waves from N=4 super Yang-Mills, JHEP0204(2002)013, hep-th/0202021

[2] S.S. Gubser, I.R. Klebanov, and A.M. Polyakov, Gauge theory correlators from noncritical string theory, Phys.Lett.B428(1998)105, hep-th/9802109,

E. Witten, Anti-de Sitter space and holography, Adv.Theor.Math.Phys.2(1998)253, hep-th/9802150

[3] For reviews, see e.g.,

A. Pankiewicz, Strings in plane wave backgrounds, Fortsch. Phys. 51 (2003) 1139.

J. C. Plefka, Lectures on the plane-wave string/gauge theory duality, hep-th/0307101.

D. Sadri and M. M. Sheikh-Jabbari, The plane-wave/super Yang-Mills duality, hep-th/0310119.

A. A. Tseytlin, Spinning strings and AdS/CFT duality, hep-th/0311139.

R. Russo and A. Tanzini, The duality between IIB string theory on PP-wave and $\mathcal{N}=4$ SYM: a status report, hep-th/0401155.

[4] S. Dobashi, H. Shimada, and T. Yoneya, Holographic reformulation of string theory on $A d S_{5} \times S^{5}$ background in the PP-wave limit, Nucl.Phys.B665(2003)94, hep-th/0209251 
[5] T. Yoneya, What is holography in the plane wave limit of $A d S(5) / S Y M(4)$ correspondence?, hep-th/0304183 (expanded from the paper published in the Proceedings, Prog. Theor. Phys. 152 (2003) 108).

[6] N. Mann and J. Polchinski, ADS holography in the Penrose limit, hep-th/0305230

[7] M. Asano, Y. Sekino, and T. Yoneya, PP wave holography for Dp-brane backgrounds, Nucl.Phys.B678(2004)197, hep-th/0308024, M. Asano and Y. Sekino, Large N limit of SYM theories with 16 supercharges from superstrings on Dp-brane backgrounds, hep-th/0405203.

[8] Y. Sekino and T. Yoneya, Generalized AdS/CFT correspondence for matrix theory in the large $N$ limit, Nucl.Phys.B570(2000)174, hep-th/9907029 Y. Sekino, Nucl. Phys. B602 (2001) 147, hep-th/0011122.

[9] E. D'Hoker, D.Z. Freedman, S.D. Mathur, A. Matusis, and L. Rastelli, Correlation functions in the $C F T_{d} / A d S_{d+1}$ correspondence, hep-th/9804058.

[10] E. D'Hoker, D.Z. Freedman, S.D. Mathur, A. Matusis, and L. Rastelli, Extremal correlators in the AdS/CFT correspondence., hep-th/9908160.

[11] S.-M. Lee, S. Minwalla, M. Rangamani, and N. Seiberg Three point functions of chiral operators in $D=4, N=4 S Y M$ at large $N$, Adv.Theor.Math.Phys.2(1998)697, hep-th/9806074

[12] T. Yoneya, see http://www2.yukawa. kyoto-u.ac.jp/ str2003/talks/yoneya.pdf

[13] E. D'Hoker, J. Erdmenger, D. Z. Freedman, and M. Pérez-Victoria, Near-extremal correlators and vanishing supergravity couplings in AdS/CFT, hep-th/0003218.

[14] C.S. Chu, V.V. Khoze, and G. Travaglini, BMN operators with vector impurities, Z(2) symmetry and pp waves, JHEP0306(2003)050, hep-th/0303107

[15] M.B. Green, J.H. Schwarz, and L. Brink, Superfield theory of type II superstring, Nucl.Phys.B219(1983)437

[16] M. Spradlin and A. Volovich, Superstring interactions in a pp wave background, Phys.Rev.D66(2002)086004,, hep-th/0204146

[17] M. Spradlin and A. Volovich, Superstring interactions in a pp wave background 2, JHEP0301(2003)036, hep-th/0206073

[18] A. Pankiewicz, More comments on superstring interactions in the pp wave background, JHEP0209(2002)056, hep-th/0208209

[19] A. Pankiewicz and B. Stefanski,Jr., PP wave light cone superstring field theory, Nucl.Phys.B657(2003)79, hep-th/0210246

[20] A. Pankiewicz, An alternative formulation of light cone string field theory on the plane wave, JHEP0306(2003)047, hep-th/0304232

[21] A. Pankiewicz and B. Stefanski,Jr., On the uniqueness of plane wave string field theory, hep-th/0308062

[22] A. Pankiewicz, strings in plane wave backgrounds, hep-th/0307027 
[23] J.H. Schwarz, Comments on superstring interactions in a plane wave background, JHEP0209(2002)058, hep-th/0208179

[24] R. Roiban, M. Spradlin, and A. Volovich, On light cone SFT contact terms in a plane wave JHEP0310(2003)055, hep-th/0211220

[25] Y. H, He, J. H. Schwarz, M. Spradlin, and A. Volovich, Explicit formulas for Neumann coefficients in the plane wave geometry, Phys.Rev.D67(2003)086005, hep-th/0211198.

See also, J. Lucietti, S. Schafer-Nameki, and A. Sinha, On the plane-wave cubic vertex, hep-th/0402185.

[26] P. Di Vecchia, J.L. Petersen, M. Petrini, R. Russo, and A. Tanzini, The three string vertex and the AdS/CFT duality in the pp wave limit, hep-th/0304025

[27] D.J. Gross, A. Mikhailov, and R. Roiban, A calculation of the plane wave string Hamiltonian from N=4 super Yang-Mills theory, JHEP0305(2003)025, hep-th/0208231

[28] C. Kristjansen, J. Plefka, G.W. Semenoff, and M. Staudacher, A new double scaling limit of $N=4$ super Yang-Mills theory and pp wave strings, Nucl.Phys.B643(2002)3, hep-th/0205033

[29] N.R. Constable, D.Z. Freedman, M.Headrick, S. Minwalla, L. Motl, A. Postnikov, and W. Skiba, PP wave string interactions from perturbative Yang-Mills theory. JHEP0207(2002)017, hep-th/0205089

[30] N. Beisert, C. Kristjansen, J. Plefka, G.W. Semenoff, and M. Staudacher, BMN correlators and operator mixing in $N=4$ super Yang-Mills theory, Nucl.Phys.B650(2003)125-161, hep-th/0208178

[31] N.R. Constable, D.Z. Freedman, M. Headrick, and S.Minwalla, Operator mixing and the BMN correspondence, JHEP0210(2002)068, hep-th/0209002

[32] J. Gomis, S. Moriyama, and J. Park SYM description of SFT Hamiltonian in a pp wave background, Nucl.Phys.B659(2003)179, hep-th/0210153

[33] J. Gomis, S. Moriyama, and J. Park SYM description of pp wave string interactions: singlet sector and arbitrary impurities, Nucl.Phys.B665(2003)49, hep-th/0301250

[34] C.S. Chu, V.V. Khoze, M. Petrini, R. Russo, and A. Tanzini, A note on string interaction on the PP wave background, Class.Quant.Grav.21(2004)1999, hep-th/0208148

[35] C.S. Chu, M. Petrini, R.Russo, and A. Tanzini String interactions and discrete symmetries of the PP wave background, Class.Quant.Grav.20(2003)S457, hep-th/0211188

[36] C.S. Chu, V.V. Khoze, Correspondence between the three point BMN correlators and the three string vertex on the pp wave, JHEP0304(2003)014, hep-th/0301036

[37] G. Georgiou and V.V. Khoze, BMN operators with three scalar impurities and the vertex correlator duality in pp wave, JHEP0304(2003)015, hep-th/0302064

[38] G. Georgiou, V.V. Khoze, and G. Travaglini, New tests of the pp wave correspondence, JHEP0310(2003)049 hep-th/0306234 
[39] G. Georgiou and G. Travaglini, Fermion BMN operators, the dilatation operator of $N=4$ SYM, and pp wave string interactions, JHEP0404(2004)001, hep-th/0403188.

[40] R. de Mello Koch, A. Donos, A. Jevicki, and J.P. Rodrigues, Derivation of string field theory from the large N BMN limit, Phys.Rev.D68(2003)065012, hep-th/0305042 